\title{
Pd-Catalyzed C-S Activation/Isocyanide Insertion/Hydrogenation Enables a Selective Aerobic Oxidation/Cyclization
}

\author{
Jian Chang ${ }^{\dagger}$, Bangyu Liu ${ }^{\dagger}$, Yang Yang ${ }^{\dagger}$, and Mang Wang* ${ }^{\dagger}, \ddagger$ \\ ${ }^{\dagger}$ Jilin Province Key Laboratory of Organic Functional Molecular Design \& Synthesis, Faculty of \\ Chemistry, Northeast Normal University, Changchun 130024, China \\ 'State Key Laboratory of Fine Chemicals, Dalian University of Technology, Dalian 116024, China
}

Table of contents

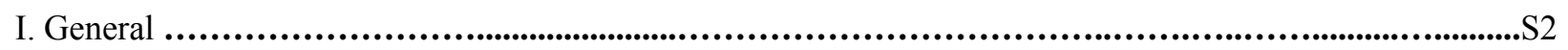

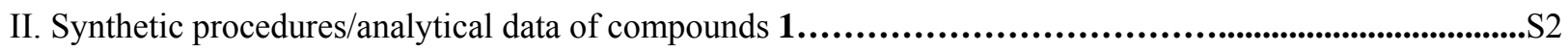

III. Synthetic procedures/analytical data of compounds 2 , 3, 4a, 5a, 6a, 7............................................S4

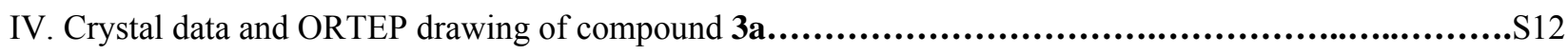

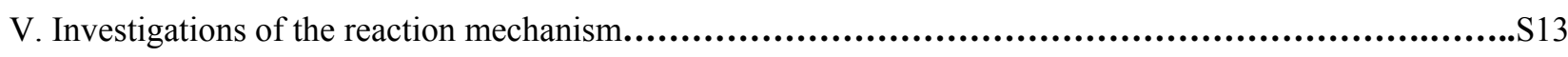

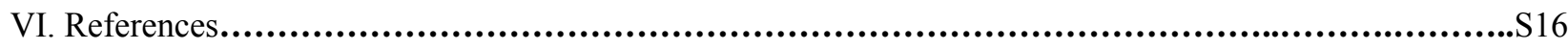

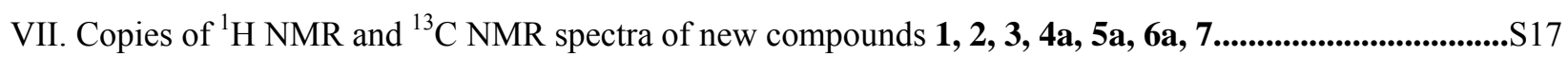




\section{General}

All reagents were purchased from commercial sources and used without treatment, unless otherwise indicated. The products were purified by column chromatography over silica gel. ${ }^{1} \mathrm{H}$ NMR and ${ }^{13} \mathrm{C}$ NMR spectra were recorded at $25^{\circ} \mathrm{C}$ on a Varian $500 \mathrm{MHz}$ or $400 \mathrm{MHz}$ and $125 \mathrm{MHz}$, and using TMS as internal standard. High-resolution mass spectra (HRMS) were obtained using a Bruker microTOF II focus spectrometer (ESI). Melting points were uncorrected. DMSO was dried over anhydrous $\mathrm{Na}_{2} \mathrm{SO}_{4}$ for $48 \mathrm{~h}$. Compound 3a with dimension $0.40 \times 0.39 \times 0.38 \mathrm{~mm}$ was glued on a glass fiber. Data were collected at $293 \mathrm{~K}$ using graphite-monochromated Mo K $\alpha$ radiation $(\lambda=0.71073 \AA)$ and Bruker APEX CCD area-detector in the range $3.00^{\circ}<\theta<25.99^{\circ}$. Substrates $\mathbf{1}$ were prepared following the known procedure. ${ }^{1} \mathbf{1 a},{ }^{2} \mathbf{1 b},{ }^{2} \mathbf{1 c},{ }^{3} \mathbf{1} \mathbf{e},{ }^{2} \mathbf{1 i},{ }^{4} \mathbf{1 j},{ }^{5} \mathbf{1 k},{ }^{6}$ 11, ${ }^{1} \mathbf{1 m},{ }^{7} \mathbf{1 n},{ }^{7} \mathbf{1 q},{ }^{8}$ are known compounds.

\section{Synthetic procedures/analytical data of compounds 1}

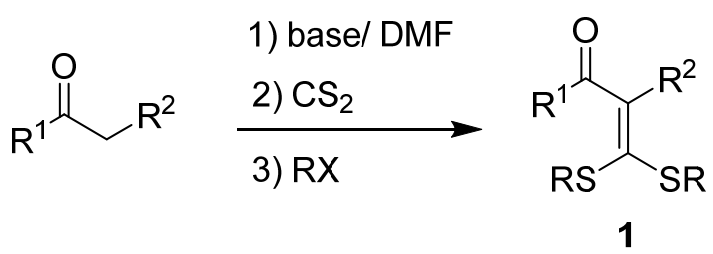

General procedure for the synthesis of ketene dithioacetals of 1a-i, 1l-q (taking 1a as an example): To a well-stirred suspension of 1-(4-fluorophenyl)propan-2-one (6.7 mL, $50 \mathrm{mmol}), \mathrm{K}_{2} \mathrm{CO}_{3}(17.2 \mathrm{~g}, 125 \mathrm{mmol})$ and DMF $(30 \mathrm{~mL})$ was stirred at room temperature for $0.5 \mathrm{~h}$. Then the reaction mixture was added $\mathrm{CS}_{2}(3.3 \mathrm{~mL}, 55$ mmol) at $0{ }^{\circ} \mathrm{C}$. After the reaction mixture was stirred at $0{ }^{\circ} \mathrm{C}$ for $1 \mathrm{~h}, \mathrm{EtBr}(8.2 \mathrm{~mL}, 110 \mathrm{mmol})$ was added dropwise within $15 \mathrm{~min}$. The mixture was allowed to warm to room temperature and stirred for $14 \mathrm{~h}$, and then poured into ice-water $(80 \mathrm{~mL})$ under stirring and extracted with $\mathrm{CH}_{2} \mathrm{Cl}_{2}(3 \times 25 \mathrm{~mL})$. The combined organic phase was washed with water $(3 \times 25 \mathrm{~mL})$, dried over anhydrous $\mathrm{MgSO}_{4}$ and concentrated in vacuo. The crude product was purified by flash chromatography (silica gel, petroleum ether/ ethyl acetate 60/1, v/v) to give 4,4-bis(ethylthio)-3-(4-fluorophenyl)but-3-en-2-one 1a (12.8 g, 90\%) as a yellow solid.

General procedure for the synthesis of ketene dithioacetals $1 \mathbf{j}$ : To a well-stirred suspension of anhydrous t-BuOK (4.94 g, $44 \mathrm{mmol})$ and butan-2-one $(1.79 \mathrm{~mL}, 20 \mathrm{mmol})$ in $40 \mathrm{~mL}$ of anhydrous DMF were added $\mathrm{CS}_{2}$ $(1.32 \mathrm{~mL}, 20 \mathrm{mmol})$ at $0{ }^{\circ} \mathrm{C}$. After the reaction mixture was stirred at $0{ }^{\circ} \mathrm{C}$ for $1.5 \mathrm{~h}$, MeI $(2.49 \mathrm{~mL}, 40 \mathrm{mmol})$ was added dropwise within $30 \mathrm{~min}$. The mixture was allowed to warm to room temperature and stirred for $5 \mathrm{~h}$, and then poured into saturated aqueous $\mathrm{NH}_{4} \mathrm{Cl}(100 \mathrm{~mL})$ under stirring. The resulting mixture was extracted with $\mathrm{CH}_{2} \mathrm{Cl}_{2}(3 \times 30 \mathrm{~mL})$. The combined organic phase was washed with water $(3 \times 15 \mathrm{~mL})$, dried over anhydrous $\mathrm{MgSO}_{4}$, filtered and concentrated in vacuo. The crude product was purified by flash chromatography (silica gel, petroleum ether/ethyl acetate: 80/1, v/v) to give 3-methyl-4,4-bis(methylthio)but-3-en-2-one $\mathbf{1 j}$ (705 $\mathrm{mg}, \mathbf{2 0 \%}$ ) as a light yellow oil.

General procedure for the synthesis of ketene dithioacetals $1 \mathbf{k}$ : To a solution of $\mathbf{1 m}(1.16 \mathrm{~g}, 5.0 \mathrm{mmol})$ in 20 $\mathrm{mL}$ of $\mathrm{CH}_{2} \mathrm{Cl}_{2}$ was added concentrated $\mathrm{H}_{2} \mathrm{SO}_{4}(1.1 \mathrm{~mL}, 20 \mathrm{mmol})$ dropwise within 15 min at $0{ }^{\circ} \mathrm{C}$. The mixture 
was allowed to warm to room temperature and stirred for $10 \mathrm{~h}$, and then poured onto saturated $\mathrm{NaCl}$ ice-water $(50$ $\mathrm{mL})$ under stirring. The mixture was neutralized with aqueous $\mathrm{NaHCO}_{3}$, and extracted with $\mathrm{CH}_{2} \mathrm{Cl}_{2}(3 \times 15 \mathrm{~mL})$. The combined organic phase was washed with water $(3 \times 10 \mathrm{~mL})$, dried over anhydrous $\mathrm{MgSO}_{4}$ and concentrated in vacuo. The crude product was purified by flash chromatography (silica gel, petroleum ether/ethyl acetate 20/1, $\mathrm{V} / \mathrm{V})$ to give 4,4-bis(ethylthio)but-3-en-2-one $\mathbf{1 k}(855 \mathrm{mg}, 90 \%)$ as a white solid.

General procedure for the synthesis of ketene dithioacetals 1r: To a stirred solution of 1c (1.48 $\mathrm{g}, 5.0 \mathrm{mmol}) \mathrm{in}$ $20 \mathrm{~mL}$ of $\mathrm{CH}_{2} \mathrm{Cl}_{2}$ was added concentrated $\mathrm{H}_{2} \mathrm{SO}_{4}(1.1 \mathrm{~mL}, 20 \mathrm{mmol})$ dropwise within $15 \mathrm{~min}$ at $0{ }^{\circ} \mathrm{C}$. The mixture was allowed to warm to room temperature and stirred for $48 \mathrm{~h}$, and then poured onto saturated $\mathrm{NaCl}$ ice-water $(50$ $\mathrm{mL})$ under stirring. The mixture was neutralized with aqueous $\mathrm{NaHCO}_{3}$, and extracted with $\mathrm{CH}_{2} \mathrm{Cl}_{2}(3 \times 15 \mathrm{~mL})$. The combined organic phase was washed with water $(3 \times 10 \mathrm{~mL})$, dried over anhydrous $\mathrm{MgSO}_{4}$ and concentrated in vacuo. The crude product was purified by flash chromatography (silica gel, petroleum ether/ ethyl acetate 100/1, V/V) to give (2-(4-methoxyphenyl)ethene-1,1-diyl)bis(ethylsulfane) 1'r (698 mg, 55\%) as a colorless liquid. Then to a stirred solution of $\mathrm{POCl}_{3}(0.18 \mathrm{~mL}, 2.0 \mathrm{mmol})$ in $5.0 \mathrm{~mL}$ anhydrous DMF was added 1'r $(254 \mathrm{mg}, 1.0 \mathrm{mmol})$ at $0{ }^{\circ} \mathrm{C}$. The mixture was allowed to warm to room temperature and stirred overnight, and then poured onto aqueous $\mathrm{K}_{2} \mathrm{CO}_{3}(15 \mathrm{~mL})$ and stirring for 20 minutes. Then the mixture was neutralized with dilute $\mathrm{HCl}$, and extracted with $\mathrm{CH}_{2} \mathrm{Cl}_{2}(3 \times 15 \mathrm{~mL})$. The combined organic phase was washed with water $(3 \times 10 \mathrm{~mL})$, dried over anhydrous $\mathrm{MgSO}_{4}$ and concentrated in vacuo. The crude product was purified by flash chromatography (silica gel, petroleum ether/ ethyl acetate 30/1, V/V) to give 3,3-bis(ethylthio)-2-(4-methoxyphenyl)acrylaldehyde 1r (225 $\mathrm{mg}, 80 \%$ ) as a yellow oil.

3-(3,4-Dimethoxyphenyl)-4,4-bis(ethylthio)but-3-en-2-one (1d)<smiles>CCSC(SCC)=C(C(C)=O)c1ccc(OC)c(OC)c1</smiles>

Yellow oil; 81\% yield (13.2 g); ${ }^{1} \mathbf{H}$ NMR (500 MHz, $\left.\mathrm{CDCl}_{3}\right) \delta 1.17(\mathrm{t}, J=7.5 \mathrm{~Hz}, 3 \mathrm{H}), 1.32(\mathrm{t}, J=7.5 \mathrm{~Hz}, 3 \mathrm{H})$, 2.30 (s, 3H), 2.68 (q, $J=7.5 \mathrm{~Hz}, 2 \mathrm{H}), 2.88$ (q, $J=7.5 \mathrm{~Hz}, 2 \mathrm{H}), 3.89$ (s, 3H), 3.90 (s, 3H), 6.86 (s, 3H). ${ }^{13} \mathbf{C ~ N M R}$ $\left(125 \mathrm{MHz}_{\mathrm{CDCl}}\right) \delta 202.0,149.2,148.8,148.5,133.5,128.3,121.8,111.7,110.5,55.8,55.7,30.3,28.3,27.9$, 14.6, 14.5. HRMS (ESI-TOF) calcd for $\mathrm{C}_{16} \mathrm{H}_{23} \mathrm{O}_{3} \mathrm{~S}_{2}^{+}\left([\mathrm{M}+\mathrm{H}]^{+}\right)$327.1083, found 327.1081 .

\section{4,4-Bis(ethylthio)-3-(2-fluorophenyl)but-3-en-2-one (1f)}<smiles>CCSC(SCC)=C(C(C)=O)c1ccccc1F</smiles>

Yellow oil; 82\% yield (11.6 g); ${ }^{1} \mathbf{H}$ NMR (500 MHz, $\left.\mathrm{CDCl}_{3}\right) \delta 1.15(\mathrm{t}, J=7.5 \mathrm{~Hz}, 3 \mathrm{H}), 1.34(\mathrm{t}, J=7.5 \mathrm{~Hz}, 3 \mathrm{H})$, $2.29(\mathrm{~s}, 3 \mathrm{H}), 2.66$ (q, $J=7.5 \mathrm{~Hz}, 2 \mathrm{H}), 2.93$ (q, $J=7.5 \mathrm{~Hz}, 2 \mathrm{H}), 7.10(\mathrm{t}, J=8.5 \mathrm{~Hz}, 1 \mathrm{H}), 7.16(\mathrm{t}, J=8.5 \mathrm{~Hz}, 1 \mathrm{H})$. $7.25(\mathrm{t}, J=8.5 \mathrm{~Hz}, 1 \mathrm{H}), 7.33(\mathrm{t}, J=8.5 \mathrm{~Hz}, 1 \mathrm{H}) .{ }^{13} \mathrm{C}$ NMR $\left(125 \mathrm{MHz}, \mathrm{CDCl}_{3}\right) \delta 199.5,159.7(\mathrm{~d}, J=244 \mathrm{~Hz}, 1 \mathrm{C})$, 144.1, 140.5, 131.4 (d, $J=2.7 \mathrm{~Hz}, 1 \mathrm{C}), 130.1$ (d, $J=7.6 \mathrm{~Hz}, 1 \mathrm{C}), 126.0$ (d, J=16.7 Hz, 1C), $124.1(\mathrm{~d}, J=3.4 \mathrm{~Hz}$, 
1C), $115.6(\mathrm{~d}, J=22.0 \mathrm{~Hz}, 1 \mathrm{C}), 30.2,28.9,28.7,14.7,14.6$. HRMS (ESI-TOF) calcd for $\mathrm{C}_{14} \mathrm{H}_{18} \mathrm{FOS}_{2}^{+}\left([\mathrm{M}+\mathrm{H}]^{+}\right)$ 285.0778, found 285.0771 .

1,1-Bis(ethylthio)-2-phenylpent-1-en-3-one (10)<smiles>CCSC(CC)=C(CC)c1ccccc1</smiles>

Light yellow oil; 76\% yield (10.6 g); ${ }^{1} \mathbf{H}$ NMR (400 MHz, $\left.\mathrm{CDCl}_{3}\right) \delta 1.07(\mathrm{t}, J=7.2 \mathrm{~Hz}, 3 \mathrm{H}), 1.15(\mathrm{t}, J=7.2 \mathrm{~Hz}$, $3 \mathrm{H}), 1.31$ (t, $J=7.2 \mathrm{~Hz}, 3 \mathrm{H}), 2.57$ (q, $J=7.2 \mathrm{~Hz}, 2 \mathrm{H}), 2.66$ (q, $J=7.2 \mathrm{~Hz}, 2 \mathrm{H}), 2.87$ (q, $J=7.2 \mathrm{~Hz}, 2 \mathrm{H})$, 7.28-7.38 (m, 5H). ${ }^{13} \mathrm{C}$ NMR (125 MHz, $\left.\mathrm{CDCl}_{3}\right) \delta$ 204.7, 150.1, 136.3, 134.1, 128.9 (2C), 128.2 (2C), 128.0, 35.9, 28.3, 27.9, 14.6, 14.5, 7.9. HRMS (ESI-TOF) calcd for $\mathrm{C}_{15} \mathrm{H}_{21} \mathrm{OS}_{2}^{+}\left([\mathrm{M}+\mathrm{H}]^{+}\right)$281.1028, found 281.1024.

3,3-Bis(ethylthio)-2-(4-methoxyphenyl)acrylaldehyde (1r)<smiles>CCC(CC)=C(C=O)c1ccc(OC)cc1</smiles>

Yellow oil; 80\% yield (225 mg) based on 1'r; ${ }^{1} \mathbf{H}$ NMR $\left(400 \mathrm{MHz}, \mathrm{CDCl}_{3}\right) \delta 1.16(\mathrm{t}, J=7.2 \mathrm{~Hz}, 3 \mathrm{H}), 1.36(\mathrm{t}, J=$ $7.2 \mathrm{~Hz}, 3 \mathrm{H}), 2.58$ (q, $J=7.2 \mathrm{~Hz}, 2 \mathrm{H}), 2.99$ (q, $J=7.2 \mathrm{~Hz}, 2 \mathrm{H}), 3.82(\mathrm{~s}, 3 \mathrm{H}), 6.93$ (t, $J=7.2 \mathrm{~Hz}, 2 \mathrm{H}), 7.15(\mathrm{t}, J=$ $7.2 \mathrm{~Hz}, 2 \mathrm{H}), 10.40$ (s, 1H). ${ }^{13} \mathrm{C}$ NMR $\left(125 \mathrm{MHz}, \mathrm{CDCl}_{3}\right) \delta 188.9,159.9,159.1,143.9,131.0$ (2C), 127.9, 113.7 (2C), 55.1, 30.2, 28.7, 14.8, 14.4. HRMS (ESI-TOF) calcd for $\mathrm{C}_{14} \mathrm{H}_{19} \mathrm{O}_{2} \mathrm{~S}_{2}{ }^{+}\left([\mathrm{M}+\mathrm{H}]^{+}\right)$283.0821, found 283.0825.

\section{Synthetic procedures/analytical data of compound 2a, 3, 4a, 5a, 6a, and 7}

General procedure for the synthesis of 2a: To a dried three-necked flask were added 1a (142 mg, $0.5 \mathrm{mmol})$, triphenylsilane (260 mg, $1.0 \mathrm{mmol}$ ), $\mathrm{Pd}\left(\mathrm{PPh}_{3}\right)_{2} \mathrm{Cl}_{2}$ (35 mg, $0.05 \mathrm{mmol}$ ), 4-isocyano-1,1'-biphenyl (269 mg, 1.5 mmol) and DMSO $(5.0 \mathrm{~mL})$, respectively, under $\mathrm{N}_{2}$. Then the mixture was heated to $90^{\circ} \mathrm{C}$ and stirred for $7 \mathrm{~h}$. After 4-isocyano-1,1'-biphenyl was consumed (monitored by TLC), the reaction mixture was poured into ice water $(30 \mathrm{~mL})$, extracted with $\mathrm{CH}_{2} \mathrm{Cl}_{2}(3 \times 15 \mathrm{~mL})$. The combined organic extracts were washed with saturated aqueous $\mathrm{NaCl}(3 \times 15 \mathrm{~mL})$, dried over anhydrous $\mathrm{MgSO}_{4}$, filtered and concentrated under reduced pressure to yield the crude product, which was purified by silica gel chromatography (eluent, petroleum ether/ ethyl acetate: $25 / 1, \mathrm{v} / \mathrm{v})$ to give $\mathbf{2 a}(90 \mathrm{mg}, 43 \%)$ as a brown solid. When $\mathrm{H}_{2} \mathrm{O}(0.5 \mathrm{~mL})$ was added, only trace amount of $2 \mathbf{a}$ was detected with the recovery of $\mathbf{1 a}$ in $95 \%$ yield.

1-([1,1'-Biphenyl]-4-yl)-3-(ethylthio)-4-(4-fluorophenyl)-5-hydroxy-5-methyl-1,5-dihydro-2H-pyrrol-2-one (2a)<smiles>CCSC1=C(c2ccc(F)cc2)C(C)(O)N(c2ccc(-c3ccccc3)cc2)C1=O</smiles> 
Brown solid; mp 148-149 ${ }^{\circ} \mathrm{C} ; 43 \%$ yield $(90 \mathrm{mg}) ;{ }^{1} \mathbf{H}$ NMR $\left(500 \mathrm{MHz}, \mathrm{CDCl}_{3}\right) \delta 1.22(\mathrm{t}, J=7.5 \mathrm{~Hz}, 3 \mathrm{H}), 1.43(\mathrm{~s}$, $3 \mathrm{H}), 3.12(\mathrm{q}, J=7.5 \mathrm{~Hz}, 2 \mathrm{H}), 3.21(\mathrm{~s}, 1 \mathrm{H}), 7.15(\mathrm{t}, J=8.0 \mathrm{~Hz}, 2 \mathrm{H}), 7.36(\mathrm{~d}, J=8.0 \mathrm{~Hz}, 1 \mathrm{H}), 7.42(\mathrm{t}, J=8.0 \mathrm{~Hz}$, 2H), $7.55(\mathrm{t}, J=8.0 \mathrm{~Hz}, 2 \mathrm{H}), 7.58(\mathrm{~s}, 4 \mathrm{H}), 7.84-7.87(\mathrm{~m}, 2 \mathrm{H}) .{ }^{13} \mathrm{C}$ NMR $\left(125 \mathrm{MHz}, \mathrm{CDCl}_{3}\right) \delta 166.9,162.9(\mathrm{~d}, J=$ $249.1 \mathrm{~Hz}, 1 \mathrm{C}), 153.8,140.1,139.4,134.4,131.1$ (d, $J=8.1 \mathrm{~Hz}, 2 \mathrm{C}), 128.8$ (2C), 127.8, 127.4, 127.3 (2C), 127.2, 126.9 (2C), $126.4(2 \mathrm{C}), 115.2(\mathrm{~d}, J=21.4 \mathrm{~Hz}, 2 \mathrm{C}), 91.9,26.1,23.4,14.9$. HRMS (ESI-TOF) calcd for $\mathrm{C}_{25} \mathrm{H}_{23} \mathrm{FNO}_{2} \mathrm{~S}^{+}\left([\mathrm{M}+\mathrm{H}]^{+}\right) 420.1428$, found 420.1423 .

\section{Synthetic procedures/analytical data of compounds 3}

General procedure for the synthesis of 2a', 3a-3j, $3 \mathbf{o}$ and $\mathbf{3 p}$ (taking $3 \mathbf{a}$ as an example): To a dried three-necked flask were added 1a (142 mg, $0.5 \mathrm{mmol})$, triphenylsilane (156 mg, $0.6 \mathrm{mmol}), \mathrm{Pd}\left(\mathrm{PPh}_{3}\right)_{2} \mathrm{Cl}_{2}(17.5 \mathrm{mg}, 0.025$ $\mathrm{mmol})$, ethyl 2-isocyanoacetate $(165 \mu \mathrm{L}, 1.5 \mathrm{mmol})$ and DMSO $(5.0 \mathrm{~mL})$, respectively, under $\mathrm{N}_{2}$. After the reaction mixture was stirred for 5 minutes at room temperature, $\mathrm{H}_{2} \mathrm{O}(0.5 \mathrm{~mL})$ was added by using a syringe. Then the mixture was heated to $70^{\circ} \mathrm{C}$ and stirred for $4 \mathrm{~h}$. After 1a was consumed (monitored by TLC), the reaction mixture was poured into ice water $(30 \mathrm{~mL})$, extracted with $\mathrm{CH}_{2} \mathrm{Cl}_{2}(3 \times 15 \mathrm{~mL})$. The combined organic extracts were washed with saturated aqueous $\mathrm{NaCl}(3 \times 15 \mathrm{~mL})$, dried over anhydrous $\mathrm{MgSO}_{4}$, filtered and concentrated under reduced pressure to yield the crude product, which was purified by silica gel chromatography (eluent, petroleum ether/ethyl acetate: $12 / 1, \mathrm{v} / \mathrm{v})$ to give $3 \mathbf{a}(152 \mathrm{mg}, 86 \%)$ as a yellow solid. Generally, the reaction proceeding for longer time or at higher reaction temperature led to the formation of by-product $5 \mathbf{a}$ in $5-10 \%$ yield or $\mathbf{6 a}$ in $10-15 \%$ yield.

General procedure for the synthesis of $\mathbf{4 a}$, $3 \mathbf{k}-\mathbf{3 n}$, 3q (taking $\mathbf{3}$ as an example): To a dried three-necked flask were added $1 \mathrm{l}(147 \mathrm{mg}, 0.5 \mathrm{mmol})$, triphenylsilane (260 mg, $1.0 \mathrm{mmol}), \mathrm{Pd}\left(\mathrm{PPh}_{3}\right)_{2} \mathrm{Cl}_{2}(35.0 \mathrm{mg}, 0.05 \mathrm{mmol})$, ethyl 2-isocyanoacetate $(165 \mu \mathrm{L}, 1.5 \mathrm{mmol})$ and DMSO $(5.0 \mathrm{~mL})$, respectively, under $\mathrm{N}_{2}$. After the reaction mixture was stirred for 5 minutes at room temperature, $\mathrm{H}_{2} \mathrm{O}(0.5 \mathrm{~mL})$ was added by using a syringe. Then the mixture was heated to $90^{\circ} \mathrm{C}$ and stirred for $4 \mathrm{~h}$. After $1 \mathrm{l}$ was consumed (monitored by TLC), the reaction mixture was poured into ice water $(30 \mathrm{~mL})$, extracted with $\mathrm{CH}_{2} \mathrm{Cl}_{2}(3 \times 15 \mathrm{~mL})$. The combined organic extracts were washed with saturated aqueous $\mathrm{NaCl}(3 \times 15 \mathrm{~mL})$, dried over anhydrous $\mathrm{MgSO}_{4}$, filtered and concentrated under reduced pressure to yield the crude product, which was purified by silica gel chromatography (eluent, petroleum ether/ethyl acetate: $15 / 1, \mathrm{v} / \mathrm{v})$ to give $3 \mathbf{3}(167 \mathrm{mg}, 92 \%)$ as a yellow solid.

Ethyl2-(4-(ethylthio)-3-(4-fluorophenyl)-2-hydroxy-2-methyl-5-oxo-2,5-dihydro-1H-pyrrol-1-yl)acetate (2a')<smiles>CCSC1=C(c2ccc(F)cc2)C(C)(O)N(Cc2ccccc2)C1=O</smiles>

Yellow oil; 35\% yield (63 mg); ${ }^{1} \mathbf{H}$ NMR (500 MHz, $\left.\mathrm{CDCl}_{3}\right) \delta 1.19(\mathrm{t}, J=7.5 \mathrm{~Hz}, 3 \mathrm{H}), 1.24(\mathrm{~s}, 3 \mathrm{H}), 2.98-3.04(\mathrm{~m}$, $1 \mathrm{H}), 3.08-3.13(\mathrm{~m}, 1 \mathrm{H}), 3.64(\mathrm{~s}, 1 \mathrm{H}), 4.51(\mathrm{~d}, J=15.5 \mathrm{~Hz}, 1 \mathrm{H}), 4.79$ (d, $J=15.5 \mathrm{~Hz}, 1 \mathrm{H}), 7.07$ (q, $J=9.0 \mathrm{~Hz}, 2 \mathrm{H})$, 7.23-7.25 (m, 1H), 7.29 (q, $J=7.5 \mathrm{~Hz}, 2 \mathrm{H}) .7 .33$ (d, $J=7.0 \mathrm{~Hz}, 2 \mathrm{H}), 7.79$ (dd, $J=5.5,8.5 \mathrm{~Hz}, 2 \mathrm{H}) .{ }^{13} \mathbf{C}$ NMR $\left(125 \mathrm{MHz}, \mathrm{CDCl}_{3}\right) \delta 167.5,162.9(\mathrm{~d}, J=248.9 \mathrm{~Hz}, 1 \mathrm{C}), 153.8,138.4,131.0(\mathrm{~d}, J=32.5 \mathrm{~Hz}, 2 \mathrm{C}), 128.5$ (2C), 
127.8 (d, $J=14.0 \mathrm{~Hz}, 1 \mathrm{C}$ ), 127.7 (2C), 127.3, 127.2, 115.3 (d, $J=85.5 \mathrm{~Hz}, 2 \mathrm{C}$ ), 90.5, 42.1, 26.0, 23.8, 15.0. HRMS (ESI-TOF) calcd for $\mathrm{C}_{20} \mathrm{H}_{20} \mathrm{FNNaO}_{2} \mathrm{~S}^{+}\left([\mathrm{M}+\mathrm{Na}]^{+}\right)$380.1091, found 380.1093.

Ethyl 2-(4-(ethylthio)-3-(4-fluorophenyl)-2-hydroxy-2-methyl-5-oxo-2,5-dihydro-1H-pyrrol-1-yl)acetate

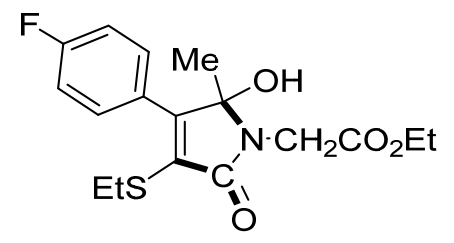

Yellow solid; mp 121-122 ${ }^{\circ} \mathrm{C}$; 86\% yield (152 mg); ${ }^{1} \mathbf{H}$ NMR (400 MHz, $\left.\mathrm{CDCl}_{3}\right) \delta 1.21(\mathrm{t}, J=7.2 \mathrm{~Hz}, 3 \mathrm{H}), 1.32(\mathrm{t}$, $J=7.2 \mathrm{~Hz}, 3 \mathrm{H}), 1.40$ (s, 3H), 3.05 (q, $J=7.2 \mathrm{~Hz}, 1 \mathrm{H}), 3.16$ (q, $J=7.2 \mathrm{~Hz}, 1 \mathrm{H}), 3.51$ (s, $1 \mathrm{H}), 3.93$ (d, $J=18.0 \mathrm{~Hz}$, 1H), 4.25 (q, $J=7.2 \mathrm{~Hz}, 2 \mathrm{H}), 4.49$ (d, $J=18.0 \mathrm{~Hz}, 1 \mathrm{H}), 7.11-7.16(\mathrm{~m}, 2 \mathrm{H}), 7.80-7.84(\mathrm{~m}, 2 \mathrm{H}) .{ }^{13} \mathbf{C}$ NMR (125 $\left.\mathrm{MHz}, \mathrm{CDCl}_{3}\right) \delta 170.8,167.4,162.9(\mathrm{~d}, J=248.7 \mathrm{~Hz}, 1 \mathrm{C}), 154.5,131.1$ (d, $\left.J=8.1 \mathrm{~Hz}, 2 \mathrm{C}\right), 127.7$ (d, $J=3.4 \mathrm{~Hz}$, 1C), 126.6, $115.2(\mathrm{~d}, J=21.0 \mathrm{~Hz}, 2 \mathrm{C}), 89.6,62.0,40.2,26.1,22.8,15.0,14.0$. HRMS (ESI-TOF) calcd for $\mathrm{C}_{17} \mathrm{H}_{20} \mathrm{FNNaO}_{4} \mathrm{~S}^{+}\left([\mathrm{M}+\mathrm{Na}]^{+}\right)$376.0989, found 376.0983.

Ethyl 2-(4-(ethylthio)-3-(4-fluorophenyl)-2-hydroxy-2-methyl-5-oxo-2,5-dihydro-1H-pyrrol-1-yl)acetate (3b)<smiles>CCOCCN1C(=O)C(CC)=C(c2ccc(Cl)cc2)C1(C)O</smiles>

Yellow solid; mp 100-101 ${ }^{\circ} \mathrm{C}$; 87\% yield (161 mg); ${ }^{1} \mathbf{H}$ NMR $\left(500 \mathrm{MHz}, \mathrm{CDCl}_{3}\right) \delta 1.21(\mathrm{t}, J=7.5 \mathrm{~Hz}, 3 \mathrm{H}), 1.31$ (t, $J=7.5 \mathrm{~Hz}, 3 \mathrm{H}), 1.39$ (s, 3H), 3.02-3.09 (m, 1H), 3.12-3.19 (m, 1H), $3.62(\mathrm{~s}, 1 \mathrm{H}), 3.94(\mathrm{~d}, J=18.0 \mathrm{~Hz}, 1 \mathrm{H}), 4.24$ (q, $J=7.5 \mathrm{~Hz}, 2 \mathrm{H}), 4.46(\mathrm{~d}, J=18.0 \mathrm{~Hz}, 1 \mathrm{H}), 7.41(\mathrm{~d}, J=8.5 \mathrm{~Hz}, 2 \mathrm{H}), 7.76(\mathrm{~d}, J=8.5 \mathrm{~Hz}, 2 \mathrm{H}) .{ }^{13} \mathbf{C}$ NMR $(125$ $\left.\mathrm{MHz}, \mathrm{CDCl}_{3}\right) \delta 171.0,167.3,154.1,135.3,130.4$ (2C), 130.1, 128.5 (2C), 127.4, 89.6, 62.1, 40.4, 26.2, 22.8, 15.1, 14.0. HRMS (ESI-TOF) calcd for $\mathrm{C}_{17} \mathrm{H}_{20} \mathrm{ClNNaO}_{4} \mathrm{~S}^{+}\left([\mathrm{M}+\mathrm{Na}]^{+}\right)$392.0694, found 392.0696 .

Ethyl 2-(4-(ethylthio)-2-hydroxy-3-(4-methoxyphenyl)-2-methyl-5-oxo-2,5-dihydro-1H-pyrrol-1-yl)acetate (3c)<smiles>CCOC(=O)CN1C(=O)C(CC)=C(c2ccc(OC)cc2)C1(C)O</smiles>

Yellow solid; mp 110-111 ${ }^{\circ} \mathrm{C} ; 80 \%$ yield $(146 \mathrm{mg}) ;{ }^{1} \mathbf{H}$ NMR $\left(400 \mathrm{MHz}, \mathrm{CDCl}_{3}\right) \delta 1.21(\mathrm{t}, J=7.2 \mathrm{~Hz}, 3 \mathrm{H}), 1.31(\mathrm{t}$, $J=7.2 \mathrm{~Hz}, 3 \mathrm{H}), 1.41$ (s, 3H), 3.03 (q, $J=7.2 \mathrm{~Hz}, 1 \mathrm{H}), 3.14$ (q, $J=7.2 \mathrm{~Hz}, 1 \mathrm{H}), 3.54$ (s, 1H), 3.85 (s, 3H), 3.94 (d, $J=18.0 \mathrm{~Hz}, 1 \mathrm{H}), 4.23(\mathrm{q}, J=7.2 \mathrm{~Hz}, 2 \mathrm{H}), 4.45(\mathrm{~d}, J=18.0 \mathrm{~Hz}, 1 \mathrm{H}), 6.96(\mathrm{dd}, J=2.0,6.8 \mathrm{~Hz}, 2 \mathrm{H}), 7.81(\mathrm{dd}, J=$ 2.0, $6.8 \mathrm{~Hz}, 2 \mathrm{H}) .{ }^{13} \mathrm{C}$ NMR (125 MHz, $\left.\mathrm{CDCl}_{3}\right) \delta 171.1,167.7,160.3,155.6,130.7$ (2C), 124.9, 124.0, 113.6 (2C), 89.7, 62.0, 55.2, 40.3, 26.3, 23.1, 15.0, 14.0. HRMS (ESI-TOF) calcd for $\mathrm{C}_{18} \mathrm{H}_{23} \mathrm{NNaO}_{5} \mathrm{~S}^{+}\left([\mathrm{M}+\mathrm{Na}]^{+}\right) 388.1189$, found 388.1174 . 
Ethyl 2-(3-(3,4-dimethoxyphenyl)-4-(ethylthio)-2-hydroxy-2-methyl-5-oxo-2,5-dihydro-1H-pyrrol-1-yl)acetate (3d)<smiles>CCOC(=O)CN1C(=O)C(CC)=C(c2ccc(OC)c(OC)c2)C1(C)O</smiles>

Light yellow solid; mp $134-135{ }^{\circ} \mathrm{C}$; 81\% yield (160 mg); ${ }^{1} \mathbf{H}$ NMR $\left(400 \mathrm{MHz}, \mathrm{CDCl}_{3}\right) \delta 1.22(\mathrm{t}, J=7.2 \mathrm{~Hz}, 3 \mathrm{H})$, 1.31 (t, $J=7.2 \mathrm{~Hz}, 3 \mathrm{H}), 1.44$ (s, 3H), 3.05 (q, $J=7.2 \mathrm{~Hz}, 1 \mathrm{H}), 3.17$ (q, $J=7.2 \mathrm{~Hz}, 1 \mathrm{H}), 3.56(\mathrm{~s}, 1 \mathrm{H}), 3.91(\mathrm{~s}, 3 \mathrm{H})$, 3.93 (s, 3H), 3.95 (d, $J=17.6 \mathrm{~Hz}, 1 \mathrm{H}), 4.24$ (q, $J=7.2 \mathrm{~Hz}, 2 \mathrm{H}), 4.48$ (d, $J=17.6 \mathrm{~Hz}, 1 \mathrm{H}), 6.93(\mathrm{~d}, J=8.4 \mathrm{~Hz}$, $1 \mathrm{H}), 7.45(\mathrm{dd}, J=2.0,8.4 \mathrm{~Hz}, 1 \mathrm{H}), 7.53(\mathrm{~d}, J=2.0 \mathrm{~Hz}, 1 \mathrm{H}) .{ }^{13} \mathbf{C}$ NMR $\left(125 \mathrm{MHz}, \mathrm{CDCl}_{3}\right) \delta 171.2,167.7,155.4$, $149.9,148.3,125.1,124.2,122.7,112.0,110.5,89.8,62.0,55.9,55.8,40.3,26.3,23.1,15.1$, 14.0. HRMS (ESI-TOF) calcd for $\mathrm{C}_{19} \mathrm{H}_{25} \mathrm{NNaO}_{6} \mathrm{~S}^{+}\left([\mathrm{M}+\mathrm{Na}]^{+}\right) 418.1295$, found 418.1289 .

Ethyl 2-(4-(ethylthio)-2-hydroxy-3-(2-methoxyphenyl)-2-methyl-5-oxo-2,5-dihydro-1H-pyrrol-1-yl)acetate (3e)<smiles>CCOC(=O)CN1C(=O)C(SC)=C(c2ccccc2OC)C1(C)O</smiles>

Light yellow solid; mp 120-121 ${ }^{\circ} \mathrm{C} ; 82 \%$ yield $(150 \mathrm{mg}) ;{ }^{1} \mathbf{H}$ NMR (500 MHz, $\left.\mathrm{CDCl}_{3}\right) \delta 1.16(\mathrm{t}, \mathrm{J}=7.5 \mathrm{~Hz}, 3 \mathrm{H})$, 1.30 (t, $J=7.5 \mathrm{~Hz}, 3 \mathrm{H}), 1.38$ (s, 3H), 2.87 (q, $J=7.5 \mathrm{~Hz}, 1 \mathrm{H}), 2.94$ (q, $J=7.5 \mathrm{~Hz}, 1 \mathrm{H}), 3.68(\mathrm{~s}, 1 \mathrm{H}), 3.83(\mathrm{~s}, 3 \mathrm{H})$, $4.04(\mathrm{~d}, J=18.0 \mathrm{~Hz}, 1 \mathrm{H}), 4.23(\mathrm{q}, J=7.5 \mathrm{~Hz}, 2 \mathrm{H}), 4.33(\mathrm{~d}, J=18.0 \mathrm{~Hz}, 1 \mathrm{H}), 6.99$ (d, $J=8.0 \mathrm{~Hz}, 1 \mathrm{H}), 7.04(\mathrm{t}, J=$ $8.0 \mathrm{~Hz}, 1 \mathrm{H}), 7.25$ (d, $J=8.0 \mathrm{~Hz}, 1 \mathrm{H}), 7.38-7.42(\mathrm{~m}, 1 \mathrm{H}) .{ }^{13} \mathbf{C}$ NMR $\left(125 \mathrm{MHz}, \mathrm{CDCl}_{3}\right) \delta 170.1,167.0,156.5$, 153.4, 130.5 (2C), 130.1, 121.0, 120.8, 111.1, 89.9, 61.6, 55.6, 40.2, 25.1, 22.4, 15.1, 14.1. HRMS (ESI-TOF) calcd for $\mathrm{C}_{18} \mathrm{H}_{23} \mathrm{NNaO}_{5} \mathrm{~S}^{+}\left([\mathrm{M}+\mathrm{Na}]^{+}\right)$388.1189, found 388.1184 .

Ethyl 2-(4-(ethylthio)-3-(2-fluorophenyl)-2-hydroxy-2-methyl-5-oxo-2,5-dihydro-1H-pyrrol-1-yl)acetate (3f)<smiles>CCOC(=O)CN1C(=O)C(CC)=C(c2ccccc2F)C1(C)O</smiles>

Yellow solid; mp 146-147 ${ }^{\circ} \mathrm{C} ; 87 \%$ yield (154 mg); ${ }^{1} \mathbf{H}$ NMR (400 MHz, CDCl 3$) \delta 1.18(\mathrm{t}, J=7.2 \mathrm{~Hz}, 3 \mathrm{H}), 1.31$ (t, $J=7.2 \mathrm{~Hz}, 3 \mathrm{H}), 1.39$ (s, 3H), 2.92-2.98 (m, 2H), $3.48(\mathrm{~s}, 1 \mathrm{H}), 3.98$ (d, $J=18.0 \mathrm{~Hz}, 1 \mathrm{H}), 4.24(\mathrm{q}, J=7.2 \mathrm{~Hz}, 2 \mathrm{H})$, $4.43(\mathrm{~d}, J=18.0 \mathrm{~Hz}, 1 \mathrm{H}), 7.14-7.24(\mathrm{~m}, 2 \mathrm{H}), 7.39-7.49$ (m, 2H). ${ }^{13} \mathbf{C}$ NMR $\left(125 \mathrm{MHz}, \mathrm{CDCl}_{3}\right) \delta$ 170.7, 167.0, $159.4(\mathrm{~d}, J=247.0 \mathrm{~Hz}, 1 \mathrm{C}), 150.5,131.2,131.0(\mathrm{~d}, J=8.1 \mathrm{~Hz}, 1 \mathrm{C}), 130.3,124.0$ (d, $J=3.4 \mathrm{~Hz}, 1 \mathrm{C}), 119.6$ (d, $J=$ $16.1 \mathrm{~Hz}, 1 \mathrm{C}), 115.8(\mathrm{~d}, J=22.0 \mathrm{~Hz}, 1 \mathrm{C}), 89.7,62.0,40.4,25.2,21.9,15.0,14.0$. HRMS (ESI-TOF) calcd for $\mathrm{C}_{17} \mathrm{H}_{20} \mathrm{FNNaO}_{4} \mathrm{~S}^{+}\left([\mathrm{M}+\mathrm{Na}]^{+}\right)$376.0989, found 376.0987.

Ethyl 2-(4-(benzylthio)-3-(4-fluorophenyl)-2-hydroxy-2-methyl-5-oxo-2,5-dihydro-1H-pyrrol-1-yl)acetate (3g) 


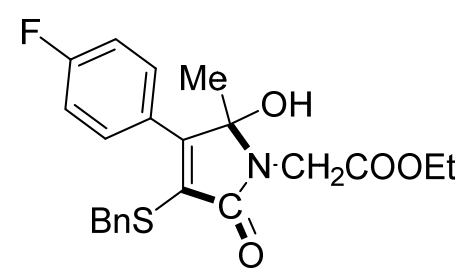

Light yellow solid; mp $138-139{ }^{\circ} \mathrm{C} ; 72 \%$ yield $(150 \mathrm{mg}) ;{ }^{1} \mathbf{H}$ NMR $\left(400 \mathrm{MHz}, \mathrm{CDCl}_{3}\right) \delta 1.29(\mathrm{~s}, 3 \mathrm{H}), 1.32(\mathrm{t}, J=$ $7.2 \mathrm{~Hz}, 3 \mathrm{H}), 3.59$ (s, 1H), $3.99(\mathrm{~d}, J=18.0 \mathrm{~Hz}, 1 \mathrm{H}), 4.13(\mathrm{~d}, J=12.8 \mathrm{~Hz}, 1 \mathrm{H}), 4.23-4.31(\mathrm{~m}, 3 \mathrm{H}), 4.42(\mathrm{~d}, J=$ $18.0 \mathrm{~Hz}, 1 \mathrm{H}), 6.99(\mathrm{t}, J=8.4 \mathrm{~Hz}, 2 \mathrm{H}), 7.17-7.25(\mathrm{~m}, 5 \mathrm{H}), 7.41(\mathrm{dd}, J=5.6,8.4 \mathrm{~Hz}, 2 \mathrm{H}) .{ }^{13} \mathrm{C} \mathbf{N M R}(125 \mathrm{MHz}$, $\left.\mathrm{CDCl}_{3}\right) \delta 170.6,167.5,163.0(\mathrm{~d}, J=249.0 \mathrm{~Hz}, 1 \mathrm{C}), 158.5,137.7,130.9(\mathrm{~d}, J=8.1 \mathrm{~Hz}, 2 \mathrm{C}), 129.1(2 \mathrm{C}), 128.3$ (2C), 127.3 (d, $J=3.4 \mathrm{~Hz}, 1 \mathrm{C}), 127.1,125.8,115.1$ (d, $J=21.4 \mathrm{~Hz}, 2 \mathrm{C}), 89.8,62.0,40.2,36.4,22.5$, 14.1. HRMS (ESI-TOF) calcd for $\mathrm{C}_{22} \mathrm{H}_{22} \mathrm{FNNaO}_{4} \mathrm{~S}^{+}\left([\mathrm{M}+\mathrm{Na}]^{+}\right)$438.1146, found 438.1151 .

Ethyl 2-(3-(4-fluorophenyl)-2-hydroxy-2-methyl-4-(methylthio)-5-oxo-2,5-dihydro-1H-pyrrol-1-yl)acetate (3h)<smiles>CCOC(=O)CN1C(=O)C(S(C)(=O)=O)=C(c2ccc(F)cc2)C1(C)O</smiles>

Light yellow solid; mp $125-126{ }^{\circ} \mathrm{C} ; 81 \%$ yield $(137 \mathrm{mg}) ;{ }^{1} \mathbf{H}$ NMR $\left(400 \mathrm{MHz}, \mathrm{CDCl}_{3}\right) \delta 1.31(\mathrm{t}, J=7.2 \mathrm{~Hz}, 3 \mathrm{H})$, 1.39 (s, 3H), 2.48 (s, 3H), 3.63 (s, 1H), 3.95 (d, $J=18.0 \mathrm{~Hz}, 1 \mathrm{H}), 4.24$ (q, $J=7.2 \mathrm{~Hz}, 2 \mathrm{H}), 4.46$ (d, $J=18.0 \mathrm{~Hz}$, $1 \mathrm{H}), 7.13(\mathrm{t}, J=8.0 \mathrm{~Hz}, 2 \mathrm{H}), 7.79(\mathrm{t}, J=8.0 \mathrm{~Hz}, 2 \mathrm{H}) .{ }^{13} \mathrm{C}$ NMR $\left(125 \mathrm{MHz}, \mathrm{CDCl}_{3}\right) \delta 171.0,167.3,163.0(\mathrm{~d}, J=$ $249.0 \mathrm{~Hz}, 1 \mathrm{C}$ ), 153.1, 131.1 (d, $J=8.1 \mathrm{~Hz}, 2 \mathrm{C}), 127.9,127.7,115.3$ (d, $J=21.5 \mathrm{~Hz}, 2 \mathrm{C}), 89.6,62.1,40.3,22.8$, 15.3, 14.0. HRMS (ESI-TOF) calcd for $\mathrm{C}_{16} \mathrm{H}_{18} \mathrm{FNNaO}_{4} \mathrm{~S}^{+}\left([\mathrm{M}+\mathrm{Na}]^{+}\right)$362.0833, found 362.0823.

Ethyl 2-(3-(4-chlorophenyl)-2-hydroxy-2-methyl-4-(methylthio)-5-oxo-2,5-dihydro-1H-pyrrol-1-yl)acetate (3i)<smiles>CCOCCN1C(=O)C(S(C)(=O)=O)=C(c2ccc(Cl)cc2)C1(C)O</smiles>

Light yellow solid; mp 119-120 ${ }^{\circ} \mathrm{C}$; 79\% yield (140 mg); ${ }^{1} \mathbf{H}$ NMR $\left(500 \mathrm{MHz}, \mathrm{CDCl}_{3}\right) \delta 1.31$ (t, $\left.J=7.5 \mathrm{~Hz}, 3 \mathrm{H}\right)$, 1.39 (s, 3H), 2.47 (s, 3H), 3.72 (s, 1H), 3.96 (d, $J=18.0 \mathrm{~Hz}, 1 \mathrm{H}), 4.23$ (q, $J=7.5 \mathrm{~Hz}, 2 \mathrm{H}), 4.42$ (d, $J=18.0 \mathrm{~Hz}$, 1H), $7.41(\mathrm{dd}, J=2.09 .0 \mathrm{~Hz}, 2 \mathrm{H}), 7.73-7.75(\mathrm{~m}, 2 \mathrm{H}) .{ }^{13} \mathrm{C}$ NMR $\left(125 \mathrm{MHz}, \mathrm{CDCl}_{3}\right) \delta 170.7,167.2,152.6,135.2$, 130.3 (2C), 130.0, 128.5 (2C), 128.4, 89.6, 62.0, 40.2, 22.7, 15.2, 14.0. HRMS (ESI-TOF) calcd for $\mathrm{C}_{16} \mathrm{H}_{18} \mathrm{ClNNaO}_{4} \mathrm{~S}^{+}\left([\mathrm{M}+\mathrm{Na}]^{+}\right)$378.0537, found 378.0533.

Ethyl 2-(2-hydroxy-2,3-dimethyl-4-(methylthio)-5-oxo-2,5-dihydro-1H-pyrrol-1-yl)acetate (3j)<smiles>CCOC(=O)CN1C(=O)C(S(C)(O)O)=C(C)C1(C)O</smiles> 
Light yellow oil; 83\% yield (108 mg); ${ }^{1} \mathbf{H}$ NMR (400 MHz, $\left.\mathrm{CDCl}_{3}\right) \delta 1.29(\mathrm{t}, J=7.2 \mathrm{~Hz}, 3 \mathrm{H}), 1.41$ (s, 3H), 2.06 (s, 3H), 3.46 (s, 3H), $3.45(\mathrm{~s}, 1 \mathrm{H}), 3.92(\mathrm{~d}, J=17.6 \mathrm{~Hz}, 1 \mathrm{H}), 4.20$ (q, $J=7.2 \mathrm{~Hz}, 2 \mathrm{H}), 4.33(\mathrm{~d}, J=17.6 \mathrm{~Hz}, 1 \mathrm{H})$.

${ }^{13} \mathrm{C}$ NMR $\left(125 \mathrm{MHz}, \mathrm{CDCl}_{3}\right) \delta 170.6,167.6,158.4,126.4,88.8,61.8,40.0,21.3,15.1,14.0,11.1$. HRMS (ESI-TOF) calcd for $\mathrm{C}_{11} \mathrm{H}_{17} \mathrm{NNaO}_{4} \mathrm{~S}^{+}\left([\mathrm{M}+\mathrm{Na}]^{+}\right)$282.0770, found 282.0774 .

Ethyl 2-(4-(ethylthio)-2-hydroxy-2-methyl-5-oxo-2,5-dihydro-1H-pyrrol-1-yl)acetate (3k)<smiles>CCOC(=O)CN1C(=O)C(SCC)=CC1(C)O</smiles>

Light yellow oil; 58\% yield (75 mg); ${ }^{1} \mathbf{H}$ NMR $\left(400 \mathrm{MHz}, \mathrm{CDCl}_{3}\right) \delta 1.28(\mathrm{t}, J=7.2 \mathrm{~Hz}, 3 \mathrm{H}), 1.37(\mathrm{t}, J=7.2 \mathrm{~Hz}$, $3 \mathrm{H}), 1.49$ (s, 3H), 2.86 (q, $J=7.2 \mathrm{~Hz}, 2 \mathrm{H}), 3.59$ (s, 1H), 3.95 (d, $J=18.0 \mathrm{~Hz}, 1 \mathrm{H}), 4.19$ (q, J = 7.2 Hz, 2H), 4.26 $(\mathrm{d}, J=18.0 \mathrm{~Hz}, 1 \mathrm{H}), 6.39$ (s, 1H). ${ }^{13} \mathrm{C}$ NMR $\left(125 \mathrm{MHz}, \mathrm{CDCl}_{3}\right) \delta 169.9,166.8,136.7,136.0,88.7,61.6,39.7$, 24.9, 22.6, 13.9, 13.2. HRMS (ESI-TOF) calcd for $\mathrm{C}_{11} \mathrm{H}_{17} \mathrm{NNaO}_{4} \mathrm{~S}^{+}\left([\mathrm{M}+\mathrm{Na}]^{+}\right)$282.0770, found 282.0771.

Ethyl 2-(3-benzoyl-4-(ethylthio)-2-hydroxy-2-methyl-5-oxo-2,5-dihydro-1H-pyrrol-1-yl)acetate (31)<smiles>CCOC(=O)CN1C(=O)C(SCC)=C(C(=O)c2ccccc2)C1(C)O</smiles>

Yellow solid; mp 142-143 ${ }^{\circ} \mathrm{C}$; $92 \%$ yield $(167 \mathrm{mg}) ;{ }^{1} \mathbf{H}$ NMR $\left(400 \mathrm{MHz}, \mathrm{CDCl}_{3}\right) \delta 1.16(\mathrm{t}, J=7.2 \mathrm{~Hz}, 3 \mathrm{H}), 1.31(\mathrm{t}$, $J=7.2 \mathrm{~Hz}, 3 \mathrm{H}), 1.62(\mathrm{~s}, 3 \mathrm{H}), 2.93(\mathrm{q}, J=7.2 \mathrm{~Hz}, 2 \mathrm{H}), 4.07$ (d, $J=17.6 \mathrm{~Hz}, 2 \mathrm{H}), 4.24(\mathrm{q}, J=7.2 \mathrm{~Hz}, 2 \mathrm{H}), 4.30$ (d, $J=17.6 \mathrm{~Hz}, 1 \mathrm{H}), 7.52(\mathrm{t}, J=7.6 \mathrm{~Hz}, 2 \mathrm{H}), 7.65(\mathrm{t}, J=7.6 \mathrm{~Hz}, 1 \mathrm{H}), 7.92(\mathrm{t}, J=7.6 \mathrm{~Hz}, 2 \mathrm{H}) .{ }^{13} \mathrm{C} \mathbf{N M R}(125 \mathrm{MHz}$, $\left.\mathrm{CDCl}_{3}\right) \delta 193.2,169.9,166.0,148.8,136.5,134.7,134.3,129.4(2 \mathrm{C}), 128.9$ (2C), 89.3, 61.9, 40.2, 25.8, 23.0, 15.0, 14.0. HRMS (ESI-TOF) calcd for $\mathrm{C}_{18} \mathrm{H}_{21} \mathrm{NNaO}_{5} \mathrm{~S}^{+}\left([\mathrm{M}+\mathrm{Na}]^{+}\right)$386.1033, found 386.1033 .

Ethyl 2-(3-acetyl-4-(ethylthio)-2-hydroxy-2-methyl-5-oxo-2,5-dihydro-1H-pyrrol-1-yl)acetate (3m)<smiles>CCCCCN1C(=O)C(CC)=C(C(C)=O)C1(C)O</smiles>

Light yellow oil; 60\% yield (90 mg); ${ }^{1} \mathbf{H}$ NMR (400 MHz, $\left.\mathrm{CDCl}_{3}\right) \delta 1.28-1.35$ (m, 6H), $1.62(\mathrm{~s}, 3 \mathrm{H}), 2.54(\mathrm{~s}, 3 \mathrm{H})$, 3.51 (q, $J=7.6 \mathrm{~Hz}, 2 \mathrm{H}), 3.80(\mathrm{~s}, 1 \mathrm{H}), 4.04$ (d, $J=18.0 \mathrm{~Hz}, 1 \mathrm{H}), 4.20-4.25(\mathrm{~m}, 3 \mathrm{H}) .{ }^{13} \mathbf{C} \mathbf{N M R}\left(125 \mathrm{MHz}, \mathrm{CDCl}_{3}\right)$ $\delta 194.8,169.2,165.0,144.4,142.9,87.8,61.8,40.0,31.1,25.3,23.5,15.5,14.0$. HRMS (ESI-TOF) calcd for $\mathrm{C}_{13} \mathrm{H}_{19} \mathrm{NNaO}_{5} \mathrm{~S}^{+}\left([\mathrm{M}+\mathrm{Na}]^{+}\right)$324.0876, found 324.0875 .

Ethyl 1-(2-ethoxy-2-oxoethyl)-4-(ethylthio)-2-hydroxy-2-methyl-5-oxo-2,5-dihydro-1H-pyrrole-3-carboxylate (3n) 
<smiles>CCOC(=O)CN1C(=O)C(CC)=C(C(=O)OCC)C1(C)O</smiles>

Light yellow oil; 65\% yield (108 mg); ${ }^{1} \mathbf{H}$ NMR (400 MHz, $\left.\mathrm{CDCl}_{3}\right) \delta 1.27-1.34(\mathrm{~m}, 6 \mathrm{H}), 1.38(\mathrm{t}, J=7.2 \mathrm{~Hz}, 3 \mathrm{H})$, 1.66 (s, 3H), 3.39 (s, 1H), 3.48 (q, J = 7.2 Hz, 2H), 4.03 (d, $J=18.0 \mathrm{~Hz}, 1 \mathrm{H}), 4.19-4.24$ (m, 3H), 4.31-4.39 (m, 2H). ${ }^{13}$ C NMR (125 MHz, $\left.\mathrm{CDCl}_{3}\right) \delta 169.2,164.7,162.7,144.4,136.4,87.4,61.7,61.2,40.0,24.5,23.0,15.5$, 14.2, 14.0. HRMS (ESI-TOF) calcd for $\mathrm{C}_{14} \mathrm{H}_{21} \mathrm{NNaO}_{6} \mathrm{~S}^{+}\left([\mathrm{M}+\mathrm{Na}]^{+}\right) 354.0982$, found 354.0981 .

Ethyl 2-(2-ethyl-4-(ethylthio)-2-hydroxy-5-oxo-3-phenyl-2,5-dihydro-1H-pyrrol-1-yl)acetate (3o)<smiles>CCOC(=O)CN1C(=O)C(SCC)=C(c2ccccc2)C1(O)OC</smiles>

Light yellow solid; mp 90-91 ${ }^{\circ} \mathrm{C} ; 77 \%$ yield (134 mg); ${ }^{1} \mathbf{H}$ NMR $\left(500 \mathrm{MHz}, \mathrm{CDCl}_{3}\right) \delta 0.53(\mathrm{t}, J=7.5 \mathrm{~Hz}, 3 \mathrm{H})$, $1.22(\mathrm{t}, J=7.5 \mathrm{~Hz}, 3 \mathrm{H}), 1.32(\mathrm{t}, J=7.5 \mathrm{~Hz}, 3 \mathrm{H}), 1.79(\mathrm{q}, J=7.5 \mathrm{~Hz}, 2 \mathrm{H}), 3.10(\mathrm{q}, J=7.5 \mathrm{~Hz}, 2 \mathrm{H}), 3.64(\mathrm{~s}, 1 \mathrm{H})$, $3.77(\mathrm{~d}, J=18.0 \mathrm{~Hz}, 1 \mathrm{H}), 4.24(\mathrm{q}, J=7.5 \mathrm{~Hz}, 2 \mathrm{H}), 4.52(\mathrm{~d}, J=18.0 \mathrm{~Hz}, 1 \mathrm{H}), 7.40-7.45(\mathrm{~m}, 3 \mathrm{H}), 7.80(\mathrm{t}, J=6.5$ $\mathrm{Hz}, 2 \mathrm{H}) .{ }^{13} \mathrm{C}$ NMR $\left(125 \mathrm{MHz}, \mathrm{CDCl}_{3}\right) \delta 171.0,168.2,154.1,131.7,129.3,128.9$ (2C), 128.1 (2C), 127.9, 92.9, 62.0, 40.3, 27.3, 26.1, 15.1, 14.0, 7.0. HRMS (ESI-TOF) calcd for $\mathrm{C}_{18} \mathrm{H}_{23} \mathrm{NNaO}_{4} \mathrm{~S}^{+}\left([\mathrm{M}+\mathrm{Na}]^{+}\right) 372.1240$, found 372.1234 .

Ethyl 2-(4-(ethylthio)-2-hydroxy-5-oxo-2,3-diphenyl-2,5-dihydro-1H-pyrrol-1-yl)acetate (3p)<smiles>CCOC(=O)CN1C(=O)C(CC)=C(c2ccccc2)C1(O)c1ccccc1</smiles>

Light yellow solid; mp 83-84 ${ }^{\circ} \mathrm{C}$; 65\% yield (129 mg); ${ }^{1} \mathbf{H}$ NMR $\left(400 \mathrm{MHz}, \mathrm{CDCl}_{3}\right) \delta 1.24(\mathrm{t}, J=5.6 \mathrm{~Hz}, 3 \mathrm{H})$, 1.27 (t, $J=5.6 \mathrm{~Hz}, 3 \mathrm{H}), 3.10-3.14(\mathrm{~m}, 2 \mathrm{H}), 3.46$ (d, $J=18.0 \mathrm{~Hz}, 1 \mathrm{H}), 4.19$ (q, $J=5.6 \mathrm{~Hz}, 2 \mathrm{H}), 4.37$ (s, $1 \mathrm{H}), 4.42$ $(\mathrm{d}, J=18.0 \mathrm{~Hz}, 1 \mathrm{H}), 7.24-7.32(\mathrm{~m}, 6 \mathrm{H}), 7.38(\mathrm{~d}, J=6.4 \mathrm{~Hz}, 2 \mathrm{H}), 7.44(\mathrm{dd}, J=2.0,7.0 \mathrm{~Hz}, 2 \mathrm{H}) .{ }^{13} \mathbf{C}$ NMR $(125$ $\left.\mathrm{MHz}, \mathrm{CDCl}_{3}\right) \delta 171.4,168.1,156.2,136.9,131.3,129.1,129.0$ (2C), 128.6, 128.5 (2C), 127.8 (2C), 127.0, 126.1 (2C), 92.0, 62.1, 41.0, 26.1, 15.2, 14.0. HRMS (ESI-TOF) calcd for $\mathrm{C}_{22} \mathrm{H}_{23} \mathrm{NNaO}_{4} \mathrm{~S}^{+}\left([\mathrm{M}+\mathrm{Na}]^{+}\right) 420.1240$, found 420.1244 .

Ethyl 2-(3-benzoyl-4-(ethylthio)-2-hydroxy-5-oxo-2-phenyl-2,5-dihydro-1H-pyrrol-1-yl)acetate (3q)<smiles>CCOC(=O)CN1C(=O)C(CC)=C(C(=O)c2ccccc2)C1(O)c1ccccc1</smiles>

Yellow solid; mp 142-143 ${ }^{\circ} \mathrm{C}$; 83\% yield (176 mg); ${ }^{1} \mathbf{H}$ NMR (400 MHz, $\left.\mathrm{CDCl}_{3}\right) \delta 1.19-1.26(\mathrm{~m}, 6 \mathrm{H}), 3.01(\mathrm{q}, J=$ $7.6 \mathrm{~Hz}, 2 \mathrm{H}), 3.63$ (d, $J=17.6 \mathrm{~Hz}, 1 \mathrm{H}), 4.17$ (q, $J=7.6 \mathrm{~Hz}, 2 \mathrm{H}), 4.35$ (d, $J=17.6 \mathrm{~Hz}, 1 \mathrm{H}), 4.77$ (s, 1H), 7.30 (m, 
$3 \mathrm{H}), 7.37-7.42(\mathrm{~m}, 4 \mathrm{H}), 7.55(\mathrm{t}, J=7.6 \mathrm{~Hz}, 1 \mathrm{H}), 7.68(\mathrm{~d}, J=7.6 \mathrm{~Hz}, 2 \mathrm{H}) .{ }^{13} \mathbf{C} \mathbf{N M R}\left(125 \mathrm{MHz}, \mathrm{CDCl}_{3}\right) \delta 192.5$, 169.9, 167.0, 148.6, 136.4, 135.9, 134.8, 134.1, 129.2 (2C), 129.0, 128.7 (4C), 126.1 (2C), 91.8, 62.0, 41.2, 25.9, 15.1, 14.0. HRMS (ESI-TOF) calcd for $\mathrm{C}_{23} \mathrm{H}_{23} \mathrm{NNaO}_{5} \mathrm{~S}^{+}\left([\mathrm{M}+\mathrm{Na}]^{+}\right) 448.1189$, found 448.1183 .

(E)-N-(tert-butyl)-2-(ethylthio)-3-(4-fluorophenyl)-4-oxopent-2-enamide (4a)<smiles>CCC(C(=O)NC(C)(C)C)=C(C=O)c1ccc(F)cc1</smiles>

Light yellow oil; 62\% yield (100 mg); ${ }^{1} \mathbf{H}$ NMR (400 MHz, $\left.\mathrm{CDCl}_{3}\right) \delta 1.22(\mathrm{t}, J=7.2 \mathrm{~Hz}, 3 \mathrm{H}), 1.45(\mathrm{~s}, 9 \mathrm{H}), 2.11$ (s, 3H), 2.73 (q, $J=7.2 \mathrm{~Hz}, 2 \mathrm{H}), 6.22(\mathrm{~s}, 1 \mathrm{H}), 7.11(\mathrm{t}, J=8.4 \mathrm{~Hz}, 2 \mathrm{H}), 7.29$ (dd, $J=5.6,8.4 \mathrm{~Hz}, 2 \mathrm{H}) .{ }^{13} \mathbf{C}$ NMR $\left(125 \mathrm{MHz}, \mathrm{CDCl}_{3}\right) \delta 198.6,164.1,162.6(\mathrm{~d}, J=247.1 \mathrm{~Hz}, 1 \mathrm{C}), 142.1,141.3,131.7,130.8(\mathrm{~d}, J=8.1 \mathrm{~Hz}, 2 \mathrm{C})$, $116.0(\mathrm{~d}, J=21.5 \mathrm{~Hz}, 2 \mathrm{C}), 52.1,29.5,28.5$ (3C), 26.9, 14.5. HRMS (ESI-TOF) calcd for $\mathrm{C}_{17} \mathrm{H}_{22} \mathrm{FNNaO}_{2} \mathrm{~S}^{+}$ $\left([\mathrm{M}+\mathrm{Na}]^{+}\right) 346.1247$, found 346.1253 .

Ethyl 2-(3-(4-fluorophenyl)-2-hydroxy-2-methyl-5-oxo-2,5-dihydro-1H-pyrrol-1-yl)acetate (5a)

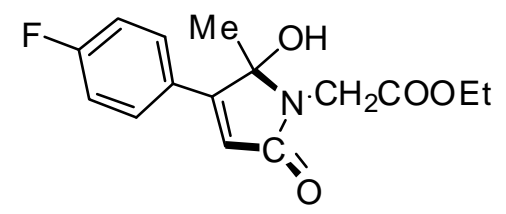

White solid; mp 169-171 ${ }^{\circ} \mathrm{C}$; $10 \%$ yield $(15 \mathrm{mg}) ;{ }^{1} \mathbf{H}$ NMR $\left(400 \mathrm{MHz}, \mathrm{CDCl}_{3}\right) \delta 1.31(\mathrm{t}, J=7.2 \mathrm{~Hz}, 3 \mathrm{H}), 1.54(\mathrm{~s}$, 3H), 3.69 (s, 1H), 3.92 (d, $J=18.0 \mathrm{~Hz}, 1 \mathrm{H}), 4.24$ (q, $J=7.2 \mathrm{~Hz}, 2 \mathrm{H}), 4.49$ (d, $J=18.0 \mathrm{~Hz}, 1 \mathrm{H}), 6.27$ (s, $1 \mathrm{H}), 7.13$ $(\mathrm{t}, J=8.8 \mathrm{~Hz}, 2 \mathrm{H}), 7.83(\mathrm{dd}, J=5.6,8.8 \mathrm{~Hz}, 2 \mathrm{H}) .{ }^{13} \mathbf{C} \mathbf{N M R}\left(125 \mathrm{MHz}, \mathrm{CDCl}_{3}\right) \delta 171.2,169.0,163.7(\mathrm{~d}, J=$ $250.0 \mathrm{~Hz}, 1 \mathrm{C}), 160.1,129.8$ (d, $J=8.1 \mathrm{~Hz}, 2 \mathrm{C}), 127.1,118.6,116.0$ (d, $J=21.4 \mathrm{~Hz}, 2 \mathrm{C}), 90.6,62.1,39.9,23.2$, 14.1. HRMS (ESI-TOF) calcd for $\mathrm{C}_{15} \mathrm{H}_{16} \mathrm{FNNaO}_{4}{ }^{+}\left([\mathrm{M}+\mathrm{Na}]^{+}\right) 316.0956$, found 316.0959 .

Ethyl 2-(3-(ethylthio)-4-(4-fluorophenyl)-5-methylene-2-oxo-2,5-dihydro-1H-pyrrol-1-yl)acetate (6a)<smiles>C=C1NC(=O)C(CCC(=O)OCC)=C1c1ccc(F)cc1</smiles>

Light yellow oil; $5 \%$ yield (9 mg); ${ }^{1} \mathbf{H}$ NMR (400 MHz, $\left.\mathrm{CDCl}_{3}\right) \delta 1.20(\mathrm{t}, J=7.2 \mathrm{~Hz}, 3 \mathrm{H}), 1.29(\mathrm{t}, J=7.2 \mathrm{~Hz}, 3 \mathrm{H})$, 3.10 (q, $J=7.2 \mathrm{~Hz}, 2 \mathrm{H}), 4.22$ (q, $J=7.2 \mathrm{~Hz}, 2 \mathrm{H}), 4.45$ (s, 2H), $4.75(\mathrm{~d}, J=2.4 \mathrm{~Hz}, 1 \mathrm{H}), 4.78(\mathrm{~d}, J=2.4 \mathrm{~Hz}, 1 \mathrm{H})$, $7.16(\mathrm{t}, J=8.4 \mathrm{~Hz}, 2 \mathrm{H}), 7.37-7.41(\mathrm{~m}, 2 \mathrm{H}) .{ }^{13} \mathrm{C}$ NMR $\left(125 \mathrm{MHz}, \mathrm{CDCl}_{3}\right) \delta 167.9,166.7,162.9(\mathrm{~d}, J=248.0 \mathrm{~Hz}$, 1C), 144.3, 143.6, 131.5 (d, $J=8.5 \mathrm{~Hz}, 2 \mathrm{C}), 128.2,126.9$ (d, $J=3.4 \mathrm{~Hz}, 1 \mathrm{C}), 115.4$ (d, $J=21.9 \mathrm{~Hz}, 2 \mathrm{C}), 94.8$, 61.6, 41.1, 25.7, 15.2, 14.1. HRMS (ESI-TOF) calcd for $\mathrm{C}_{17} \mathrm{H}_{19} \mathrm{FNO}_{3} \mathrm{~S}^{+}\left([\mathrm{M}+\mathrm{H}]^{+}\right) 336.1064$, found 336.1070.

General procedure for the synthesis of $\mathbf{7}$ (taking $\mathbf{7 a}$ as an example): To a dried sealed tube were added $\mathbf{3 l}$ (109 $\mathrm{mg}, 0.3 \mathrm{mmol}), \mathrm{Pd}\left(\mathrm{PPh}_{3}\right)_{4}(34.7 \mathrm{mg}, 0.03 \mathrm{mmol})$, CuTc $(86 \mathrm{mg}, 0.45 \mathrm{mmol})$, phenylboronic acid (73 $\mathrm{mg}, 0.6$ $\mathrm{mmol}$ ) and THF $(3.0 \mathrm{~mL})$, respectively, under $\mathrm{N}_{2}$ at room temperature. Then the mixture was heated to $80^{\circ} \mathrm{C}$ and 
stirred for $32 \mathrm{~h}$. The reaction mixture was poured into ice water $(30 \mathrm{~mL})$, extracted with $\mathrm{CH}_{2} \mathrm{Cl}_{2}(3 \times 10 \mathrm{~mL})$. The combined organic extracts were dried over anhydrous $\mathrm{MgSO}_{4}$, filtered and concentrated under reduced pressure to yield the crude product, which was purified by silica gel chromatography (eluent, petroleum ether/ethyl acetate: 12/1, v/v) to give ethyl 2-(3-benzoyl-2-hydroxy-2-methyl-5-oxo-4-phenyl-2,5-dihydro-1H-pyrrol-1-yl)acetate 7a (77 $\mathrm{mg}, 68 \%)$ as a white solid.

Ethyl 2-(3-benzoyl-2-hydroxy-2-methyl-5-oxo-4-phenyl-2,5-dihydro-1H-pyrrol-1-yl)acetate (7a)<smiles>CCOC(=O)CN1C(=O)C(c2ccccc2)=C(C(=O)c2ccccc2)C1(C)O</smiles>

White solid; mp 69-70 ${ }^{\circ} \mathrm{C}$; $68 \%$ yield $(77 \mathrm{mg}) ;{ }^{1} \mathbf{H}$ NMR $\left(400 \mathrm{MHz}, \mathrm{CDCl}_{3}\right) \delta 1.33(\mathrm{t}, J=7.2 \mathrm{~Hz}, 3 \mathrm{H}), 1.71$ (s, $3 \mathrm{H}), 3.87(\mathrm{~s}, 1 \mathrm{H}), 4.11$ (d, $J=18.0 \mathrm{~Hz}, 1 \mathrm{H}), 4.26$ (q, $J=7.2 \mathrm{~Hz}, 2 \mathrm{H}), 4.46$ (d, $J=18.0 \mathrm{~Hz}, 1 \mathrm{H}), 7.19$ (t, $J=7.2 \mathrm{~Hz}$, $3 \mathrm{H}), 7.30(\mathrm{t}, J=8.0 \mathrm{~Hz}, 2 \mathrm{H}), 7.44-7.48(\mathrm{~m}, 3 \mathrm{H}), 7.77(\mathrm{~d}, J=8.0 \mathrm{~Hz}, 2 \mathrm{H}) .{ }^{13} \mathbf{C} \mathbf{N M R}\left(125 \mathrm{MHz}, \mathrm{CDCl}_{3}\right) \delta 194.5$, 170.6, 167.5, 150.5, 135.3, 134.3, 134.2, 129.5 (2C), 129.3129 .5 (2C), 129.0, 128.6 (2C), 128.2 (2C), 88.7, 62.0, 40.3, 22.8, 14.1. HRMS (ESI-TOF) calcd for $\mathrm{C}_{22} \mathrm{H}_{21} \mathrm{NNaO}_{5}^{+}\left([\mathrm{M}+\mathrm{Na}]^{+}\right) 402.1312$, found 402.1300 .

Ethyl 2-(3-benzoyl-4-(4-chlorophenyl)-2-hydroxy-2-methyl-5-oxo-2,5-dihydro-1H-pyrrol-1-yl)acetate (7b)<smiles>CCOC(=O)CN1C(=O)C(c2ccc(Cl)cc2)=C(C(=O)c2ccccc2)C1(C)O</smiles>

White solid; mp 84-85 ${ }^{\circ} \mathrm{C}$; $65 \%$ yield $(81 \mathrm{mg}) ;{ }^{1} \mathbf{H}$ NMR $\left(500 \mathrm{MHz}, \mathrm{CDCl}_{3}\right) \delta 1.34(\mathrm{t}, J=7.5 \mathrm{~Hz}, 3 \mathrm{H}), 1.70$ (s, 3H), 3.93 (s, 1H), 4.08 (d, $J=18.0 \mathrm{~Hz}, 1 \mathrm{H}), 4.27$ (q, $J=7.5 \mathrm{~Hz}, 2 \mathrm{H}), 4.46$ (d, $J=18.0 \mathrm{~Hz}, 1 \mathrm{H}), 7.17$ (d, $J=8.5$ $\mathrm{Hz}, 2 \mathrm{H}), 7.34$ (t, $J=8.5 \mathrm{~Hz}, 2 \mathrm{H}), 7.44(\mathrm{~d}, J=8.0 \mathrm{~Hz}, 2 \mathrm{H}), 7.51(\mathrm{t}, J=8.0 \mathrm{~Hz}, 1 \mathrm{H}), 7.78(\mathrm{~d}, J=8.0 \mathrm{~Hz}, 2 \mathrm{H}) .{ }^{13} \mathrm{C}$ NMR $\left(125 \mathrm{MHz}, \mathrm{CDCl}_{3}\right) \delta 194.3,170.6,167.2,151.1,135.7,135.2,134.5,132.8,130.5$ (2C), 129.5 (2C), 128.8 (2C), 128.6 (2C), 127.5, 88.7, 62.2, 40.3, 22.7, 14.1. HRMS (ESI-TOF) calcd for $\mathrm{C}_{22} \mathrm{H}_{20} \mathrm{ClNNaO}_{5}^{+}\left([\mathrm{M}+\mathrm{Na}]^{+}\right)$ 436.0922, found 436.0922 .

\section{Crystal data and ORTEP drawing of compound 3a}

Crystal data for 3a: $\mathrm{C}_{17} \mathrm{H}_{20} \mathrm{FNO}_{4} \mathrm{~S}$, colorless, $\mathrm{M}=353.40$, triclinic, space group $\mathrm{P}-1, \mathrm{a}=8.5007(9) \AA, \mathrm{b}=$ 10.2500(12) $\AA, c=11.2985(15) \AA, V=896.65(18) \AA^{3}, \alpha=75.804(10), \beta=70.345(11), \gamma=81.631(9), Z=2, T=$ 293(2) K, F000 = 372, 5666 reflections collected, 3499 unique with $\mathrm{R}($ int $)=0.0206, \mathrm{R}^{1}=0.0532, \mathrm{wR}^{2}=0.1505$ $(\mathrm{I}>2 \sigma(\mathrm{I}))$. CCDC 1042264 contains the supplementary crystallographic data for this paper. These data can be obtained free of charge from The Cambridge Crystallographic Data Centre via www.ccdc.cam.ac.uk/data_request/cif. 

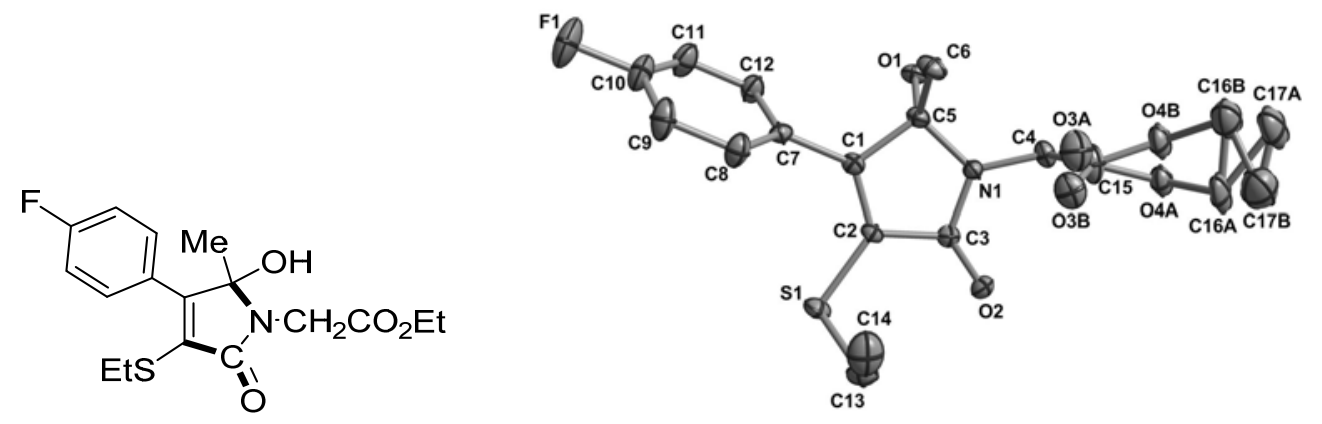

Figure. ORTEP diagram of $3 \mathbf{a}$.

\section{Investigations of the reaction mechanism}

To demonstrate the role of both $\mathrm{H}_{2} \mathrm{O}$ and $\mathrm{O}_{2}$, the model reaction was performed with or without $\mathrm{H}_{2} \mathrm{O}$ or $\mathrm{O}_{2}$, respectively. ${ }^{18} \mathrm{O}$-Labeled experiments were also included for identifying where the oxygen atom in the amide carbonyl of $\gamma$-lactam 3a originated.

\section{The role of $\mathrm{H}_{2} \mathrm{O}$ for the reaction:}

(a) To a sealed tube were added 1a $(28.2 \mathrm{mg}, 0.1 \mathrm{mmol})$, triphenylsilane (31.2 $\mathrm{mg}, 0.12 \mathrm{mmol}), \mathrm{Pd}_{(}\left(\mathrm{PPh}_{3}\right)_{2} \mathrm{Cl}_{2}$ (3.5 mg, $0.005 \mathrm{mmol})$, ethyl 2-isocyanoacetate $(33 \mu \mathrm{L}, 0.3 \mathrm{mmol})$ and dried DMSO $(1.0 \mathrm{~mL})$ with $4 \AA \mathrm{MS}$, respectively, under $\mathrm{N}_{2}$. Then the mixture was heated at $70{ }^{\circ} \mathrm{C}$ for $4 \mathrm{~h}$. Almost no reaction was detected under TLC.

(b) To a sealed tube were added 1a $(28.2 \mathrm{mg}, 0.1 \mathrm{mmol})$, triphenylsilane (31.2 $\mathrm{mg}, 0.12 \mathrm{mmol}), \mathrm{Pd}_{(}\left(\mathrm{PPh}_{3}\right)_{2} \mathrm{Cl}_{2}$ (3.5 mg, $0.005 \mathrm{mmol}$ ), ethyl 2-isocyanoacetate ( $33 \mu \mathrm{L}, 0.3 \mathrm{mmol}$ ) and DMSO (1.0 mL), respectively, under $\mathrm{N}_{2}$. After the reaction mixture was stirred for 5 minutes at room temperature, $\mathrm{H}_{2} \mathrm{O}(0.1 \mathrm{~mL})$ was added by using a syringe. Then the mixture was heated to $70{ }^{\circ} \mathrm{C}$ and stirred for $4 \mathrm{~h}$. 1a was detected to be consumed almost completely but no 3a was observed under TLC at this time. Then the reaction was quenched by $0.5 \mathrm{~mL}$ of water under $\mathrm{N}_{2}$ and stirred for additional $1 \mathrm{~h}$. No $3 \mathrm{a}$ was detected under TLC, too.

The above experimental results could demonstrate that water plays a role for Pd-catalzyed cross-coupling of 1a with isocyanide, but has no effect on the final formation of $\gamma$-lactam 3a from the reaction intermediate.

\section{The role of $\mathrm{O}_{2}$ for the reaction:}

(1) To a sealed tube were added 1a $(28.2 \mathrm{mg}, 0.1 \mathrm{mmol})$, triphenylsilane (31.2 $\left.\mathrm{mg}, 0.12 \mathrm{mmol}), \mathrm{Pd}_{(\mathrm{PPh}}\right)_{2} \mathrm{Cl}_{2}$ (3.5 mg, $0.005 \mathrm{mmol}$ ), ethyl 2-isocyanoacetate $(33 \mu \mathrm{L}, 0.3 \mathrm{mmol})$ and DMSO $(1.0 \mathrm{~mL})$, respectively, under $\mathrm{N}_{2}$. After the reaction mixture was stirred for 5 minutes at room temperature, $\mathrm{H}_{2} \mathrm{O}(0.1 \mathrm{~mL})$ was added by using a syringe. Then the mixture was heated to $70{ }^{\circ} \mathrm{C}$ and stirred for $4 \mathrm{~h}$ for the consumption of 1a. The resulting reaction mixture was stirred under $\mathrm{O}_{2}$ for additional $1 \mathrm{~h}$. 3a was detected to be formed along with a consumption of a corresponding intermediate under TLC. The isolated yield of 3a was $75 \%$.

(2) To a sealed tube were added 1a $(28.2 \mathrm{mg}, 0.1 \mathrm{mmol})$, triphenylsilane (31.2 $\left.\mathrm{mg}, 0.12 \mathrm{mmol}), \mathrm{Pd}_{(} \mathrm{PPh}_{3}\right)_{2} \mathrm{Cl}_{2}$ (3.5 mg, $0.005 \mathrm{mmol}$ ), ethyl 2-isocyanoacetate ( $33 \mu \mathrm{L}, 0.3 \mathrm{mmol})$ and DMSO $(1.0 \mathrm{~mL})$, respectively, under $\mathrm{O}_{2}$. After the reaction mixture was stirred for 5 minutes at room temperature, $\mathrm{H}_{2} \mathrm{O}(0.1 \mathrm{~mL})$ was added by using a syringe. Then the mixture was heated to $70{ }^{\circ} \mathrm{C}$ and stirred for $4 \mathrm{~h}$. The reaction occurred and 3a could be observed under TLC. However, the isolated yield of $3 \mathbf{a}$ is less than $20 \%$ with the recovery of $\mathbf{1 a}$ in $50 \%$.

The above experimental results could demonstrate that $\mathrm{O}_{2}$ plays a role for the final formation of $\gamma$-lactam 3a from a corresponding intermediate, but has a bad effect on Pd-catalyzed cross-coupling of $\mathbf{1 a}$ with isocyanide. 


\section{3. ${ }^{18} \mathrm{O}$-Labled experiments:}

(a) $\mathrm{H}_{2}{ }^{18} \mathrm{O}$-Labled experiment: To a sealed tube were added 1a $(28.2 \mathrm{mg}, 0.1 \mathrm{mmol})$, triphenylsilane $(31.2 \mathrm{mg}$, $0.12 \mathrm{mmol}), \mathrm{Pd}\left(\mathrm{PPh}_{3}\right)_{2} \mathrm{Cl}_{2}(3.5 \mathrm{mg}, 0.005 \mathrm{mmol})$, ethyl 2-isocyanoacetate (33 $\left.\mu \mathrm{L}, 0.3 \mathrm{mmol}\right)$ and DMSO $(1.0 \mathrm{~mL})$, respectively, under $\mathrm{N}_{2}$. After the reaction mixture was stirred for 5 minutes at room temperature, $\mathrm{H}_{2}{ }^{18} \mathrm{O}(0.1 \mathrm{~mL})$ was added by using a syringe. Then the mixture was heated to $70{ }^{\circ} \mathrm{C}$ and stirred for $4 \mathrm{~h}$ for the consumption of $1 \mathrm{a}$. After the exchange of $\mathrm{N}_{2}$ by ${ }^{16} \mathrm{O}_{2}$, the resulting reaction mixture was stirred under ${ }^{16} \mathrm{O}_{2}$ for additional $1 \mathrm{~h}$. Samples were then diluted with $\mathrm{MeCN}$ prior to the injection into the mass spectrometer. Only ${ }^{16} \mathrm{O}-3 \mathrm{a}$ was detected by HRMS. Additional experiment of the above reaction by quenching with $0.2 \mathrm{~mL}$ of $\mathrm{H}_{2}{ }^{18} \mathrm{O}$ did not yet afford ${ }^{18} \mathrm{O}-3 \mathbf{a}$.

(b) ${ }^{18} \mathrm{O}_{2}$-Labled experiment: To a sealed tube were added $\mathbf{1 a}(28.2 \mathrm{mg}, 0.1 \mathrm{mmol})$, triphenylsilane $(31.2 \mathrm{mg}$, $0.12 \mathrm{mmol}), \mathrm{Pd}\left(\mathrm{PPh}_{3}\right)_{2} \mathrm{Cl}_{2}(3.5 \mathrm{mg}, 0.005 \mathrm{mmol})$, ethyl 2-isocyanoacetate (33 $\left.\mu \mathrm{L}, 0.3 \mathrm{mmol}\right)$ and DMSO (1.0 mL), respectively, under $\mathrm{N}_{2}$. After the reaction mixture was stirred for 5 minutes at room temperature, $\mathrm{H}_{2}{ }^{16} \mathrm{O}(0.1 \mathrm{~mL})$ was added by using a syringe. Then the mixture was heated to $70^{\circ} \mathrm{C}$ and stirred for $4 \mathrm{~h}$ for the consumption of $\mathbf{1 a}$. After the exchange of $\mathrm{N}_{2}$ by ${ }^{18} \mathrm{O}_{2}$, the resulting reaction mixture was stirred under ${ }^{18} \mathrm{O}_{2}$ for additional $1 \mathrm{~h}$. Samples were then diluted with $\mathrm{MeCN}$ prior to the injection into the mass spectrometer. Pseudomolecular ion of ${ }^{18} \mathrm{O}-\mathbf{3 a}$ was detected in HRMS spectra $\left(\mathrm{m} / \mathrm{z}=378.1000, \mathrm{M}+2+\mathrm{Na}^{+}\right)$. The above experimental results could demonstrate that the oxygen atom in the amide carbonyl of $\gamma$-lactam 3a originated from $\mathrm{O}_{2}$ in the air.

\section{HRMS spectrum of the reaction mixture for the ${ }^{18} \mathrm{O}_{2}$-labeled experiment:}

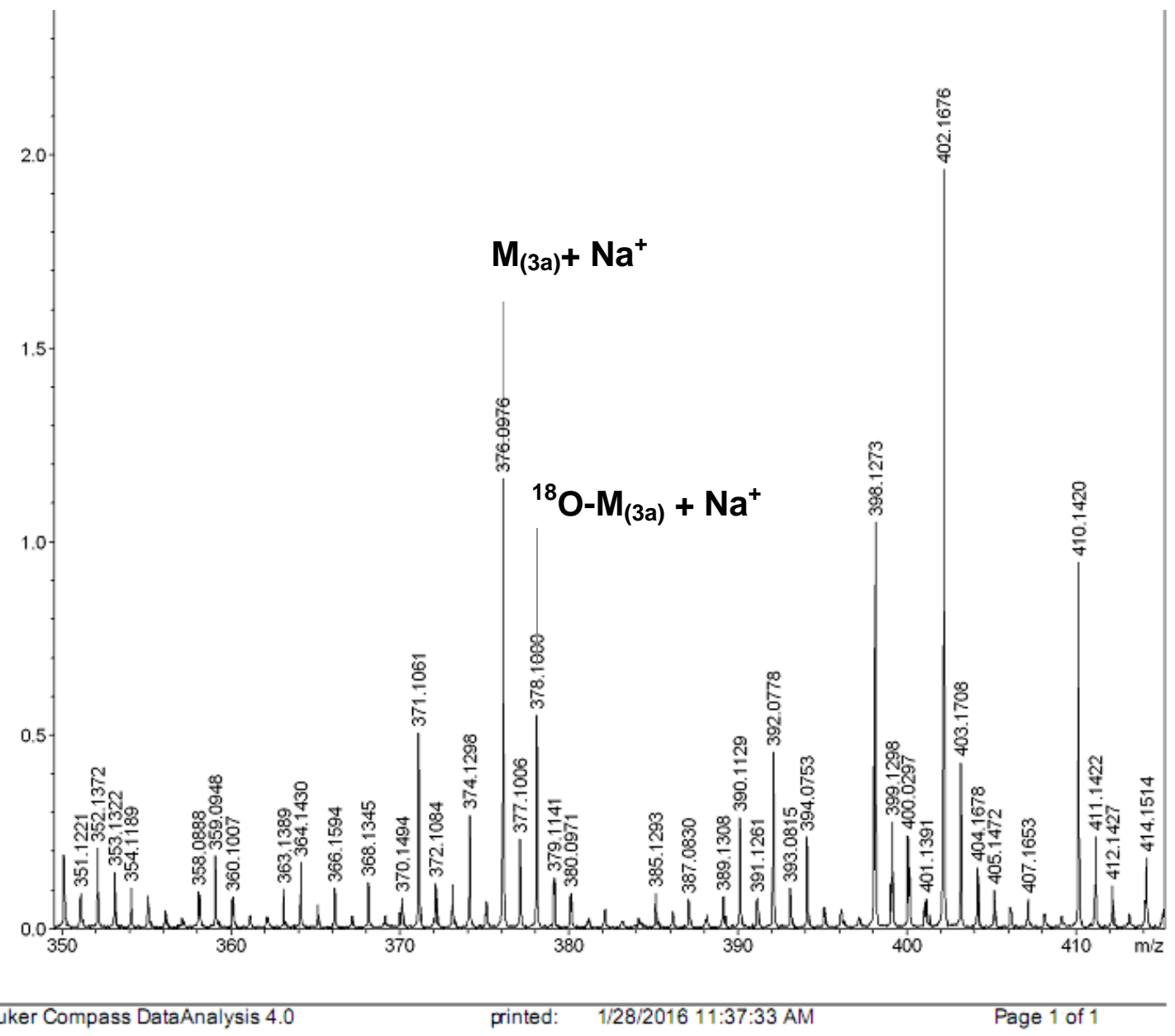


4. HRMS-ESI study of the reaction mixture: To a sealed tube were added 1a (28.2 $\mathrm{mg}, 0.1 \mathrm{mmol})$, triphenylsilane $(31.2 \mathrm{mg}, 0.12 \mathrm{mmol}), \mathrm{Pd}\left(\mathrm{PPh}_{3}\right)_{2} \mathrm{Cl}_{2}(3.5 \mathrm{mg}, 0.005 \mathrm{mmol})$, ethyl 2-isocyanoacetate $(33 \mu \mathrm{L}, 0.3$ mmol) and DMSO (1.0 mL), respectively, under $\mathrm{N}_{2}$. After the reaction mixture was stirred for 5 minutes at room temperature, $\mathrm{H}_{2} \mathrm{O}(0.1 \mathrm{~mL})$ was added by using a syringe. Then the mixture was heated to $70^{\circ} \mathrm{C}$ and stirred for $4 \mathrm{~h}$ for the consumption of 1a. Samples were diluted with MeCN prior to the injection into the mass spectrometer. It was found, among the obtained spectra, that a peak at $\mathrm{m} / \mathrm{z}=504.2585$ could be regarded as B in HRMS spectrum 1 , and the peaks at $\mathrm{m} / \mathrm{z}=338.1220$ and 360.1037 could be regarded as $\mathbf{D}$ in MS spectrum 2.<smiles>CCOCCN=C([Se]CC)C(CC)=C(C(C)=O)c1ccc(F)cc1</smiles>

B $\left(\mathrm{M}+\mathrm{H}^{+}\right)$, calculated: 504.0289

\section{HRMS spectrum 1:}

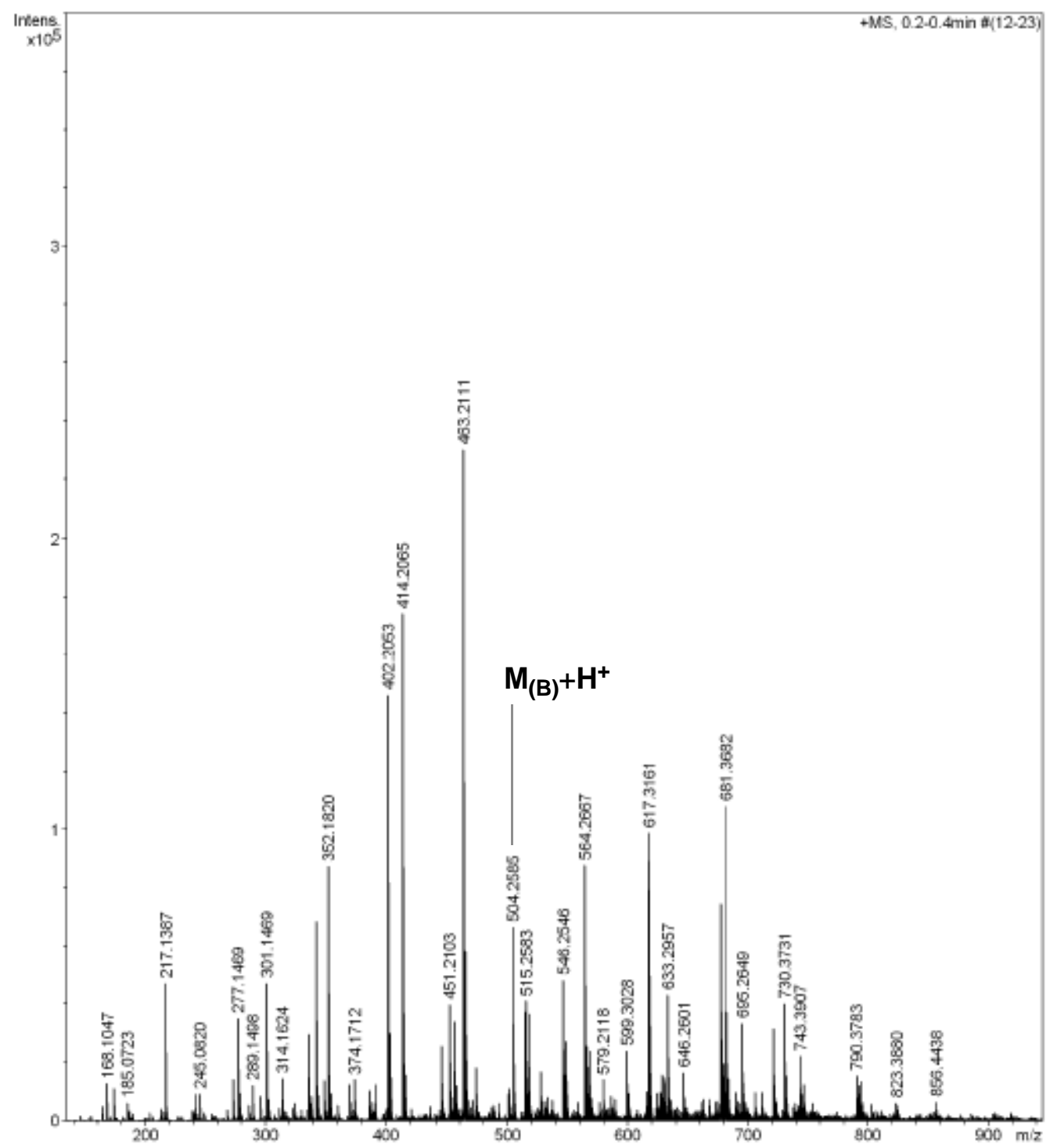


<smiles>CCOC(=O)C=NC(=O)/C(C(C)=O)=C(/C(C)=O)c1ccc(F)cc1</smiles>

D $\left(\mathrm{M}+\mathrm{H}^{+}\right)$, calculated: 338.1221

D $\left(\mathrm{M}+\mathrm{Na}^{+}\right)$, calculated: 360.1041

\section{HRMS spectrum 2:}

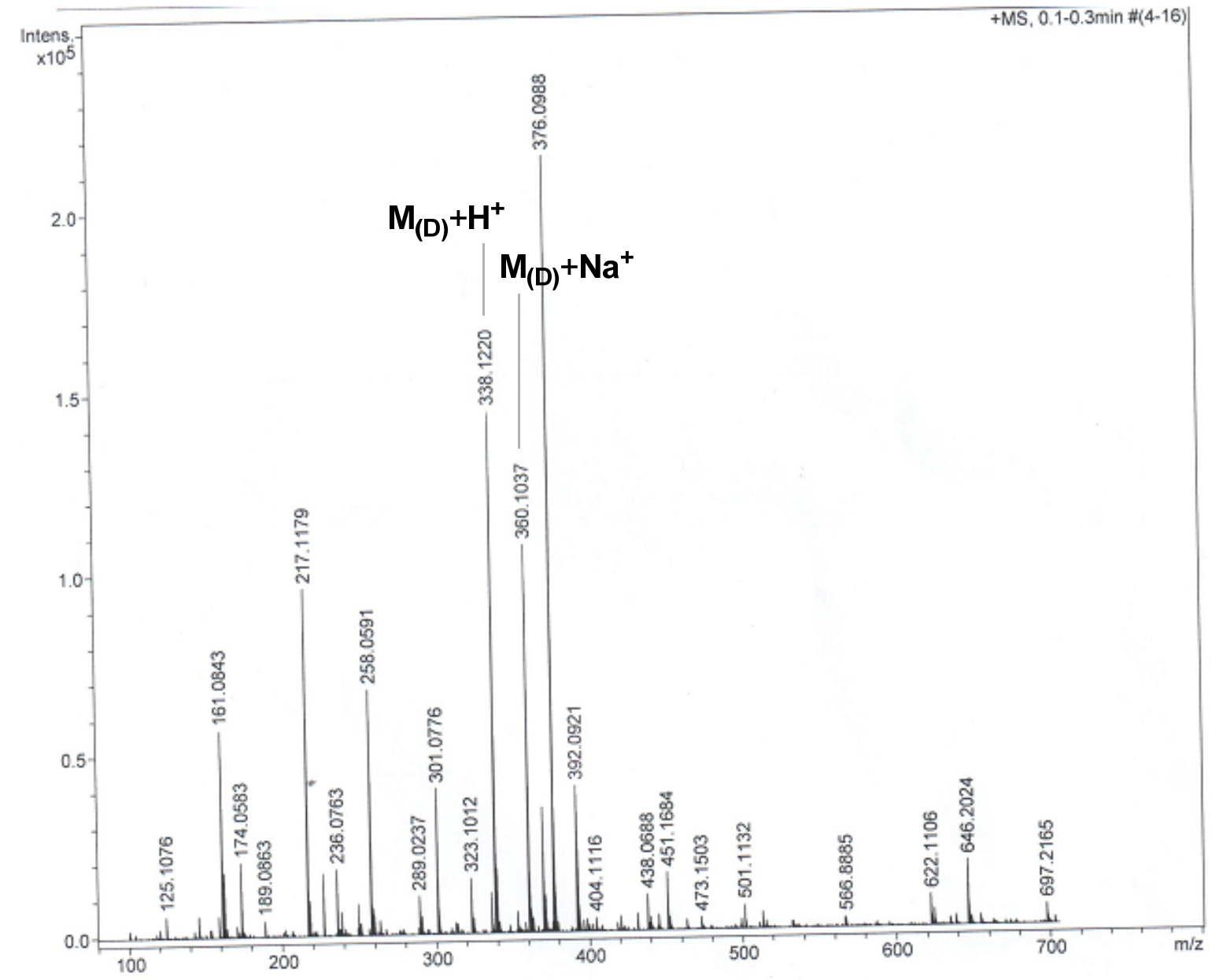

\section{References}

(1) (a) Bi, X.; Dong, D.; Liu, Q.; Pan, W.; Zhao, L.; Li, B. J. Am. Chem. Soc. 2005, 127, 4578. (b) Liu, J.; Wang, M.; Li, B.; Liu, Q.; Zhao, Y. J. Org. Chem. 2007, 72, 4401.

(2) Liu, B.; Zheng, G.; Liu, X.; Xu, C.; Liu, J.; Wang, M. Chem. Commun. 2013, 49, 2201.

(3) Zhang, L.; Liang, F.; Cheng, X.; Liu, Q. J. Org. Chem. 2009, 74, 899.

(4) Chauhan, S. M. S.; Junjappa, H. Tetrahedron 1976, 32, 1779.

(5) Thuillier, A.; Viable, J. Bull. Soc. Chim. Fr. 1962, 2187.

(6) Sun, S.; Liu, Y.; Liu, Q.; Zhao, Y.; Dong, D. Synlett. 2004, 1731.

(7) Yin, Y.; Xu, Q.; Yin, G. J. Jiangxi Normal Univ., Nat. Sci. ED 2012, 36, 412.

(8) Mashraqui, S. H.; Hariharasubrahmanian, H. J. Chem. Res., Synop. 1999, 492.

VII. Copies of ${ }^{1} \mathrm{H}$ NMR and ${ }^{13} \mathrm{C}$ NMR spectra of new compounds $\mathbf{1}, \mathbf{2 a}, \mathbf{3}, \mathbf{4 a}, \mathbf{5 a}, \mathbf{6 a}, 7$. 
STANOARO PROTON FARAMETERS

Archive directory: /export/thome/ouyy/vinirsys, da:
Sampie directory:

pulse sequence: s2pu

Solvent: Coclis

Thove fBos "menus 00 -

Relax. de lay $1.000 \mathrm{se}$
Pulse 45.0 degrees

Acci time 1.892 sec

OBSERVE H1, 499.8025802 HHz

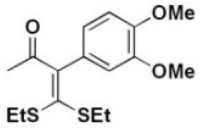

Tol
Total it ime 65536 min, $11 \mathrm{sec}$
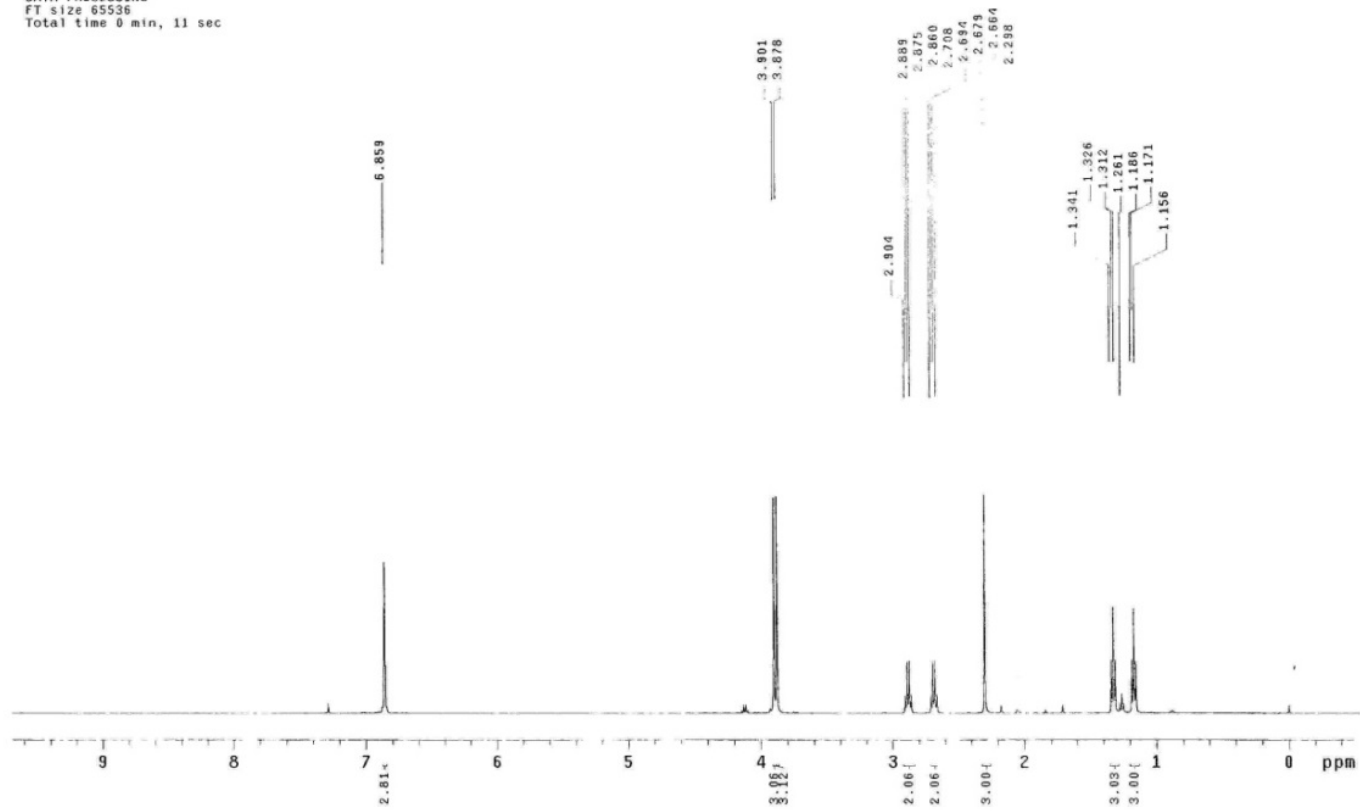

andard Carbon parameters

Archive directory: /export/home/ouyy/vner sys/data
Sample directory:

Pulse Sequence: $: 2$ pul

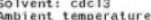

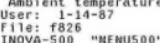

Relax. delay 0.500 sec

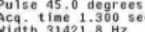

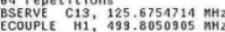

Power 42 de
cont
continous 1 ,

WAtT2-16 modulated
WATA PROCESSTM
DAT

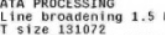

size 131072
fotal time $3 \mathrm{hr}, 56 \mathrm{sec}$

$\mid$
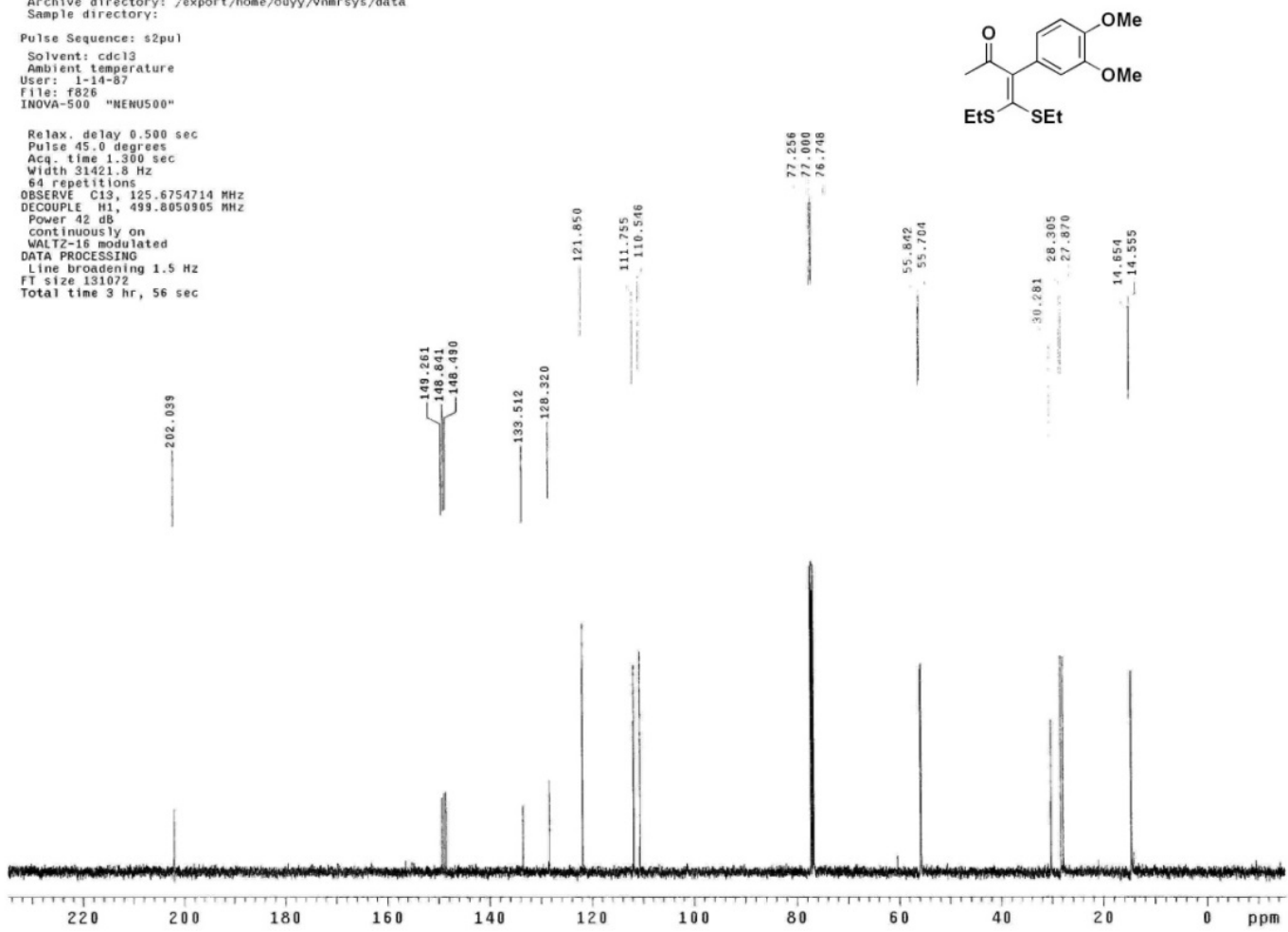
Std Proton paraneters

Sample directory:

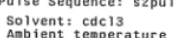

Ambient temp
operator: 1 pq

Relax. delay $1.000 \mathrm{sec}$
Pulse 45.0 degrees

Aide time 6398.998 Hz

OBSERVE H1, 399.9615226 MHI

DATA PROCESSING
TYS Size 32768 .
Total time 0 min, 50 sec

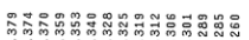

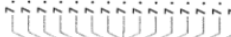

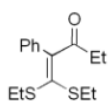

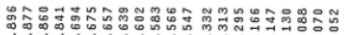

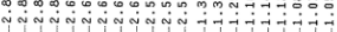

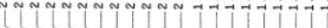

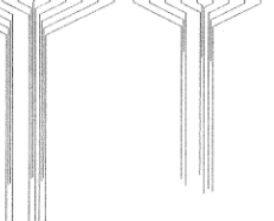

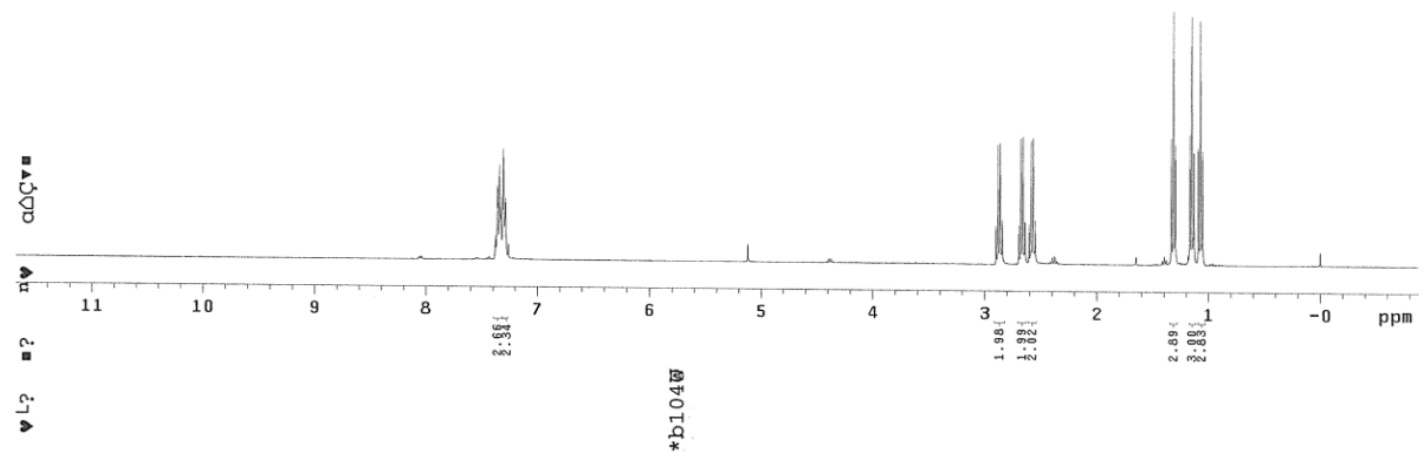

STANDARD CARBON PARAMETERS

Archive directory: /export/home/ouyy/vnmrsys/data
Sample directory:

Pulse Sequence: s2pu

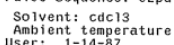

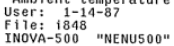

Relax. delay $0.500 \mathrm{sec}$
Pulse 45.0 degrees
Acq. time 1.300 sec
With $3141.8 \mathrm{~Hz}$

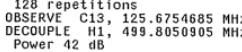

Cont inuous ly on
WALTZ-16

Line broadening $1.5 \mathrm{~Hz}$
Fize 131072 . $56 \mathrm{sec}$
Total time $3 \mathrm{hr}, 56 \mathrm{sec}$
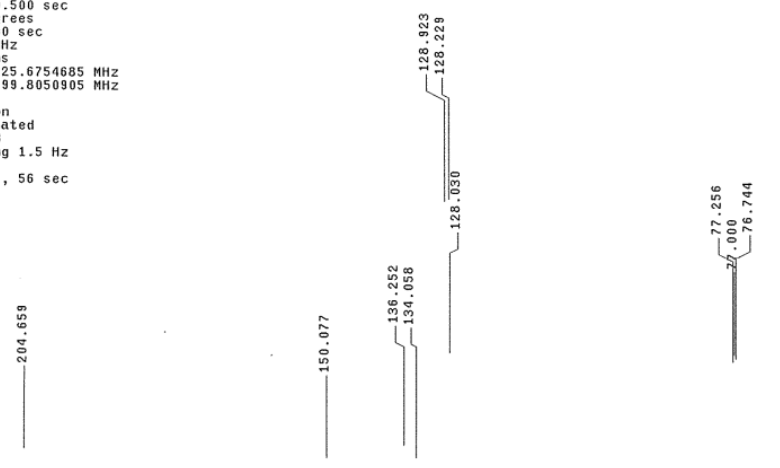

$$
{ }_{E t S} \overbrace{S E t}^{O}
$$
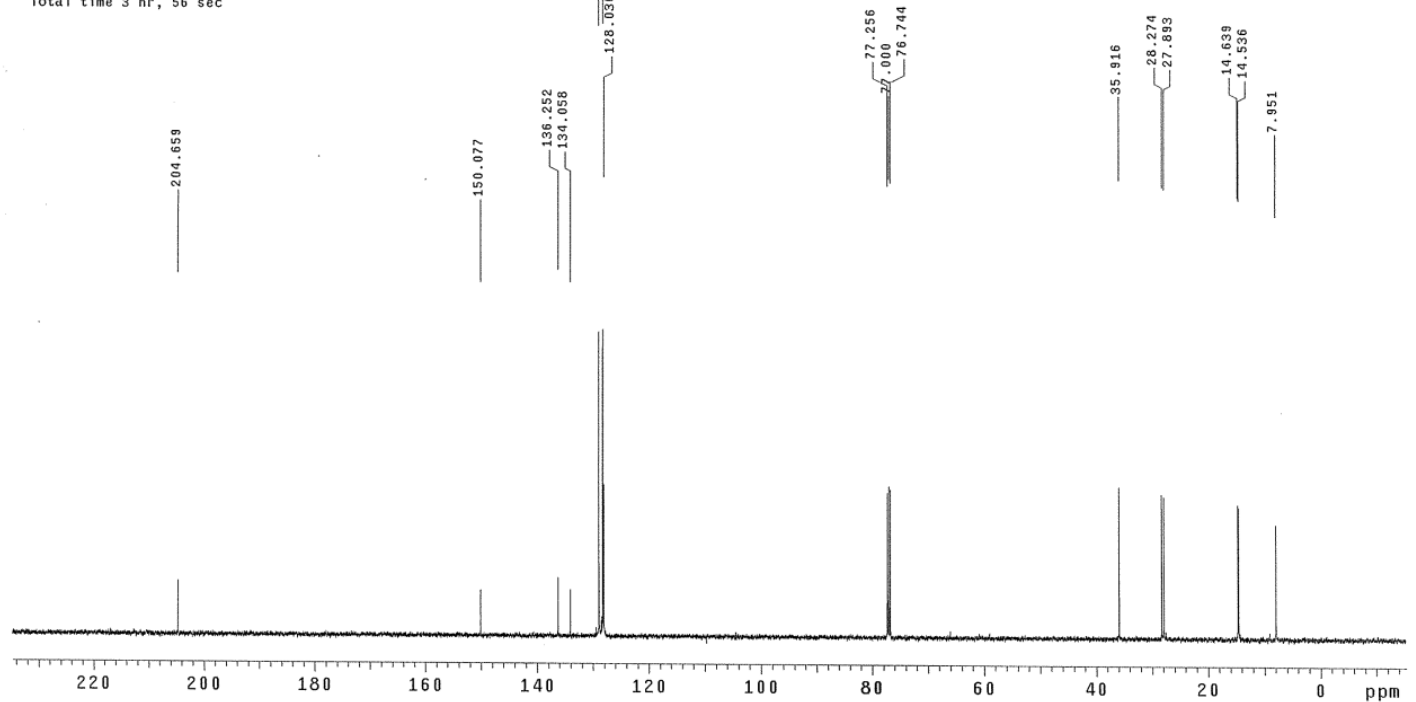
Std Proton parameters

Sample directory:

Pulse Sequence: s2pu

Aolvent: cdclis
Ambient temperat
operator: 1 pq

Fife: 7351
Mercury -4008 "varian"

Relax. delay 1.000 se
Pulse 45.0 degrees

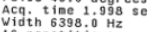

OBSERVE H1 $399.9648592 \mathrm{MHz}$

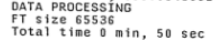

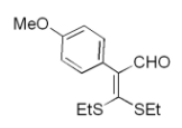

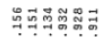

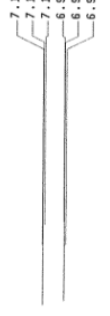

ฐ

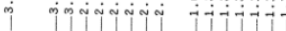

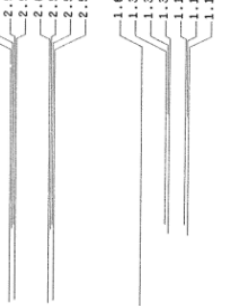

13

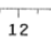

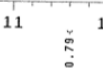

$10 \quad 9$
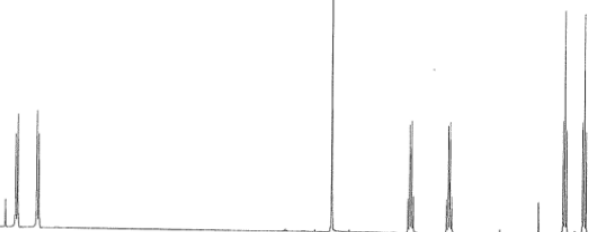

管要

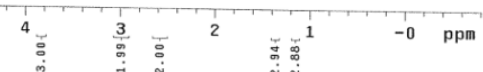

Archive directory: /export/home/ouyy/vnmrsys/data
Sample di rectory:

Pulse Sequence: s2pu

Solvent: cdcl3
Amb ient temperature

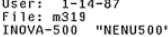

Relax. delay $0.500 \mathrm{sec}$
Pulse 45.0 degrees

Acq. time $1.300 \mathrm{sec}$
Width $31421.8 \mathrm{~Hz}$

64 repetitions
OBSERVE C13, 125.6754690 MH
DSCOUP H1; 499.8050905 MH

Power 42 dB
PB

WATA PROCESSING
DAT

FT size 131072 .
Total time $2 \mathrm{hr}, 3 \mathrm{~min}, 31$

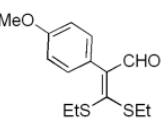

Total time $2 \mathrm{hr}, 3 \mathrm{~min}, 31$ בec

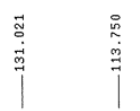<smiles>C#CCCCCC#N</smiles>

EtS ${ }_{\text {SEt }}$

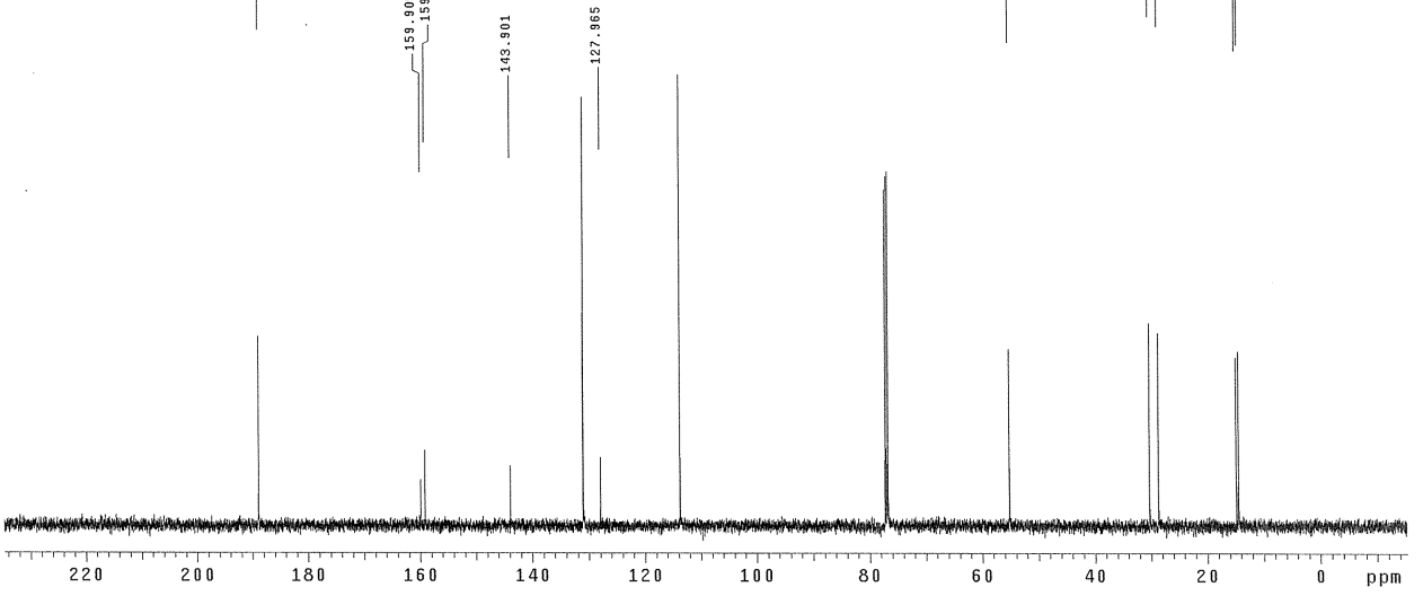




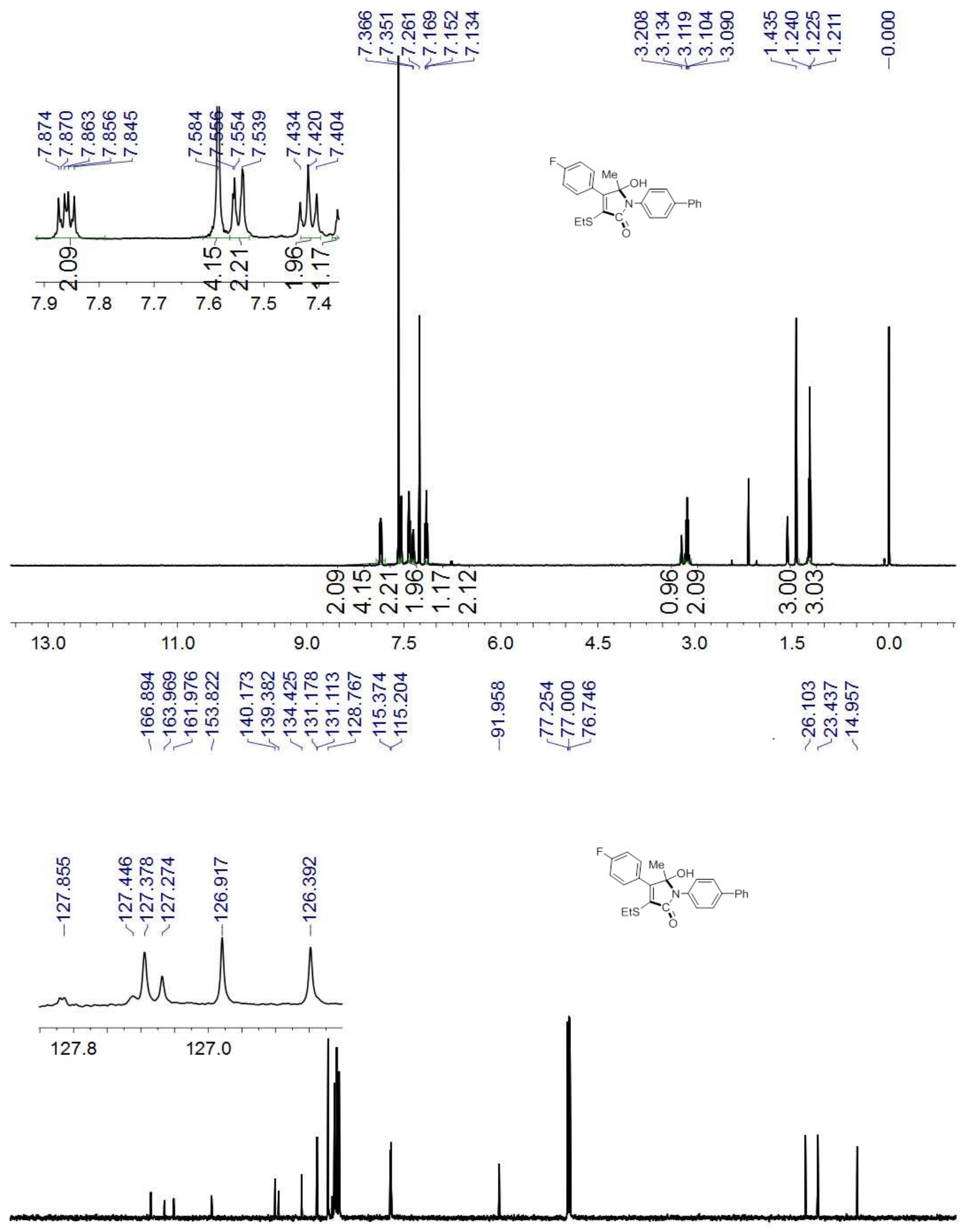

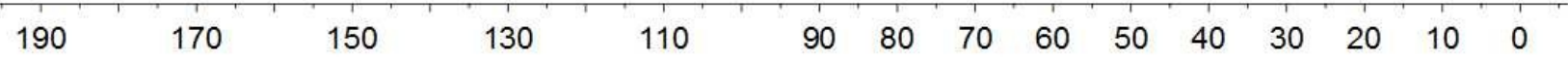




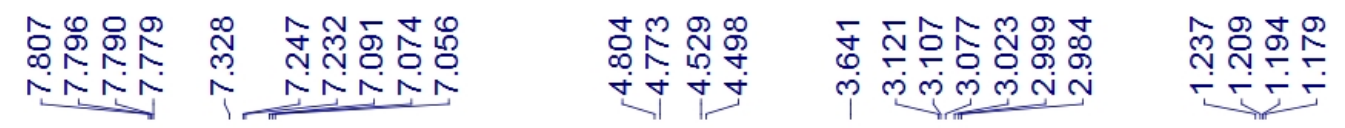
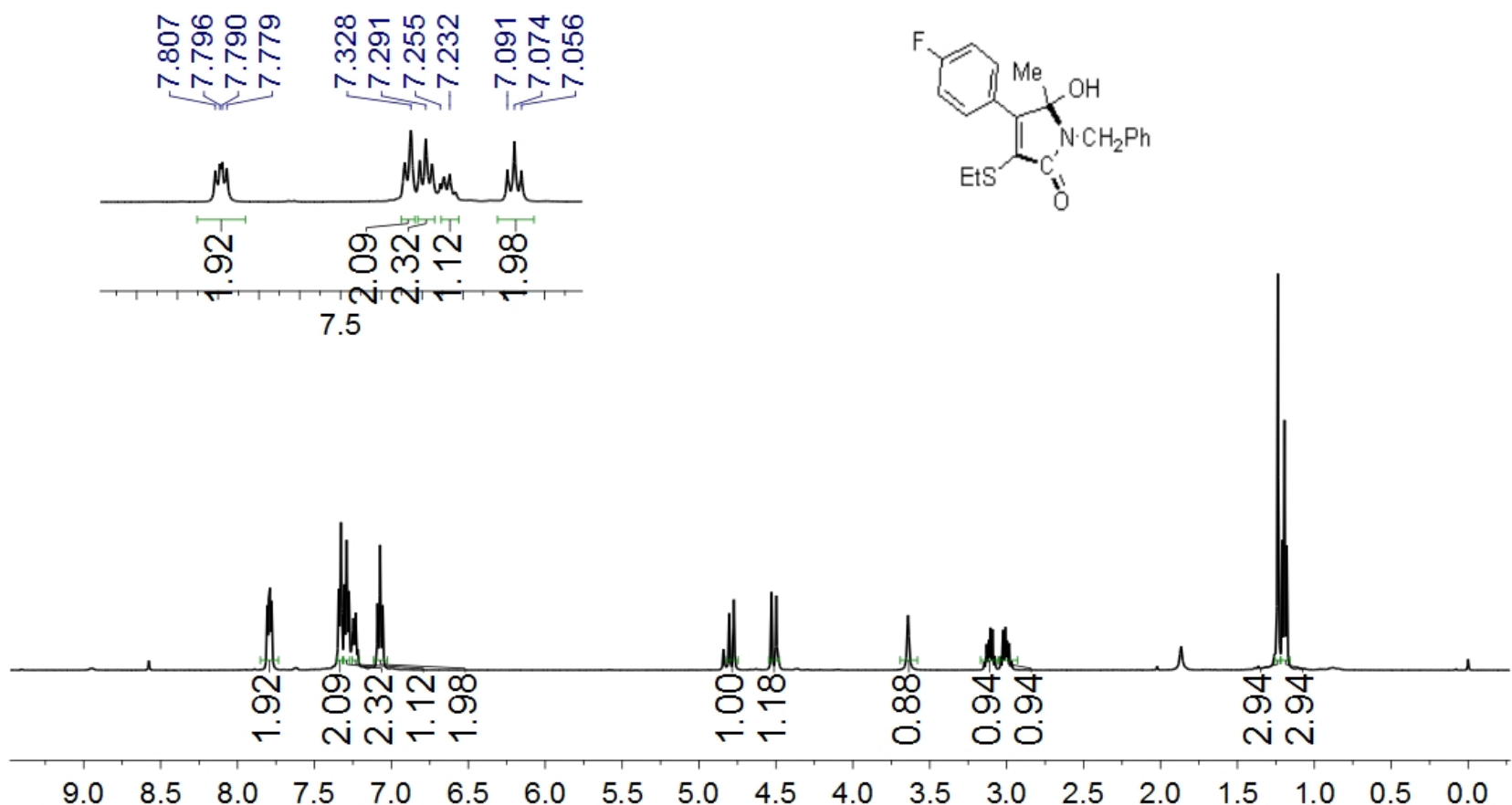

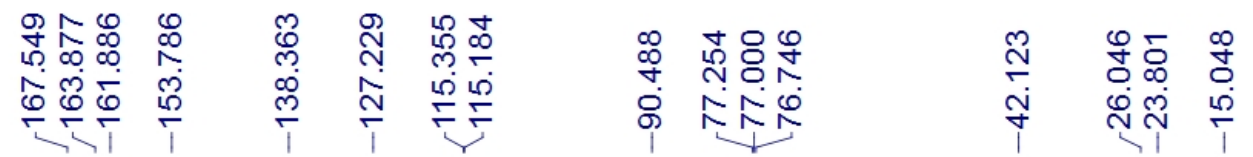
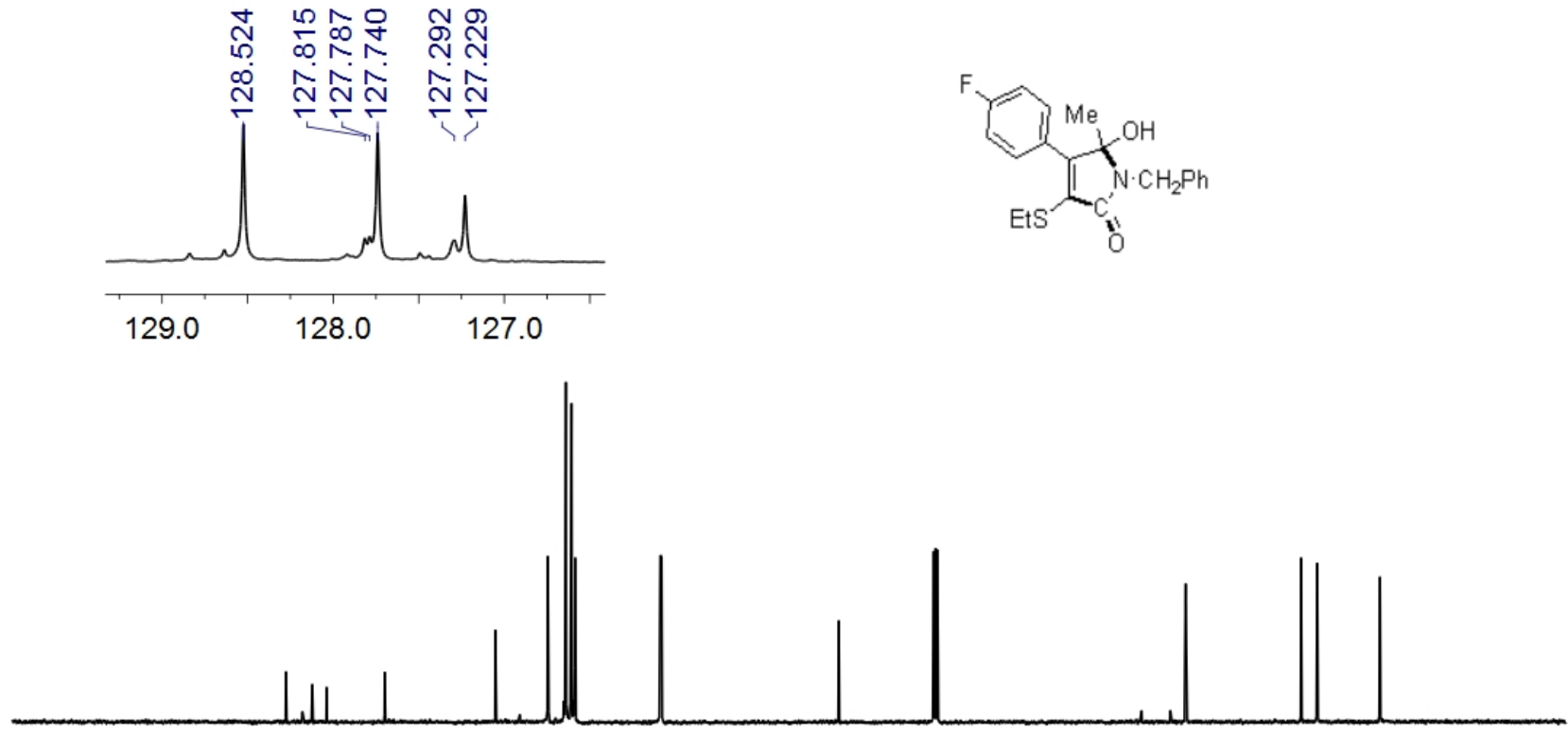

190

$170 \quad 150$

130

110

$90 \quad 80 \quad 70$

$2010 \quad 0$ 


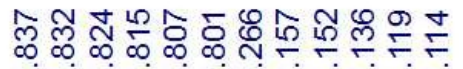
NNNNNNNNNNN
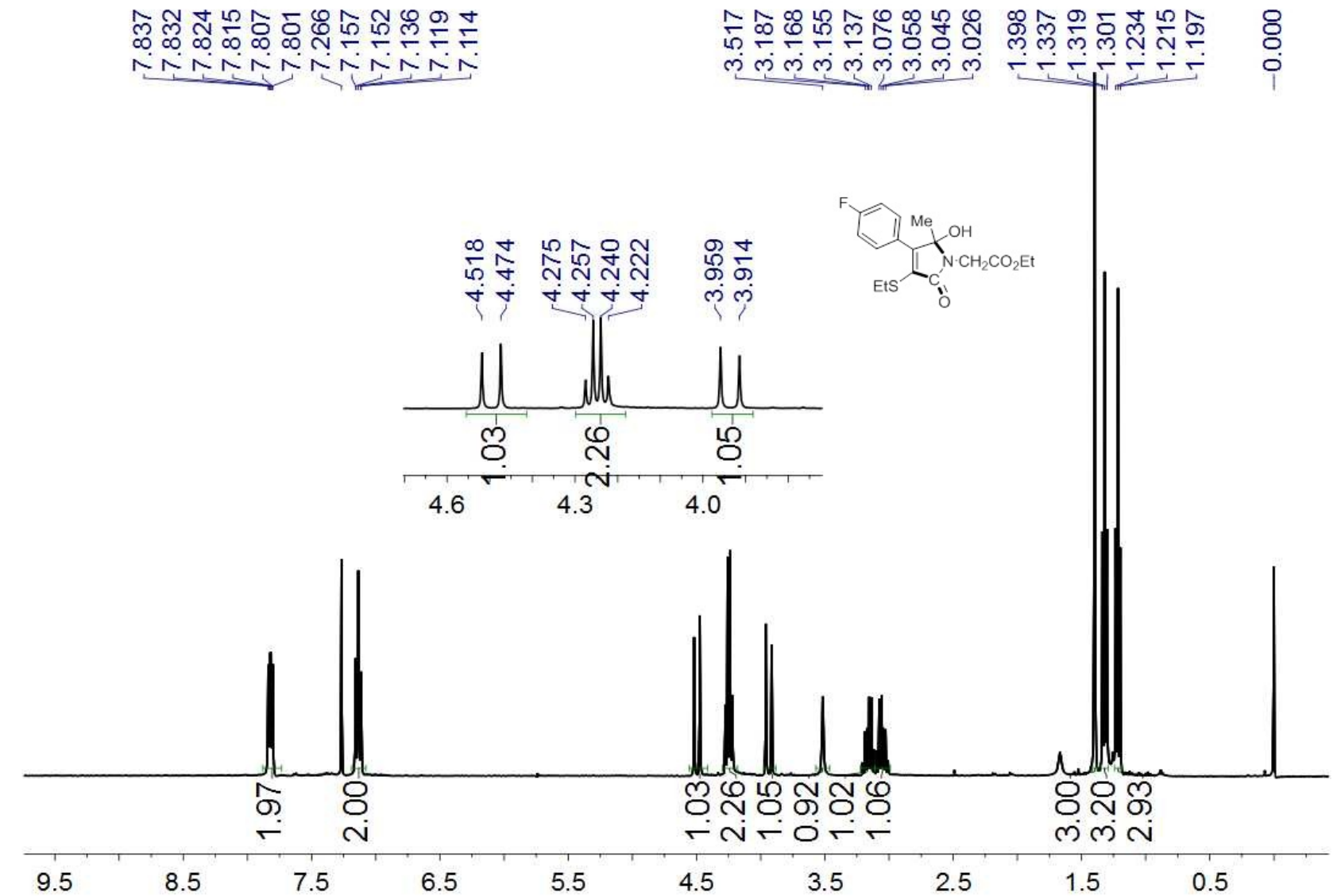

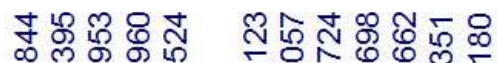

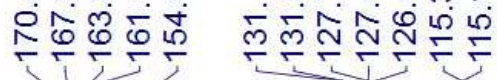

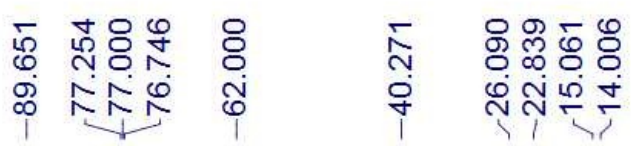

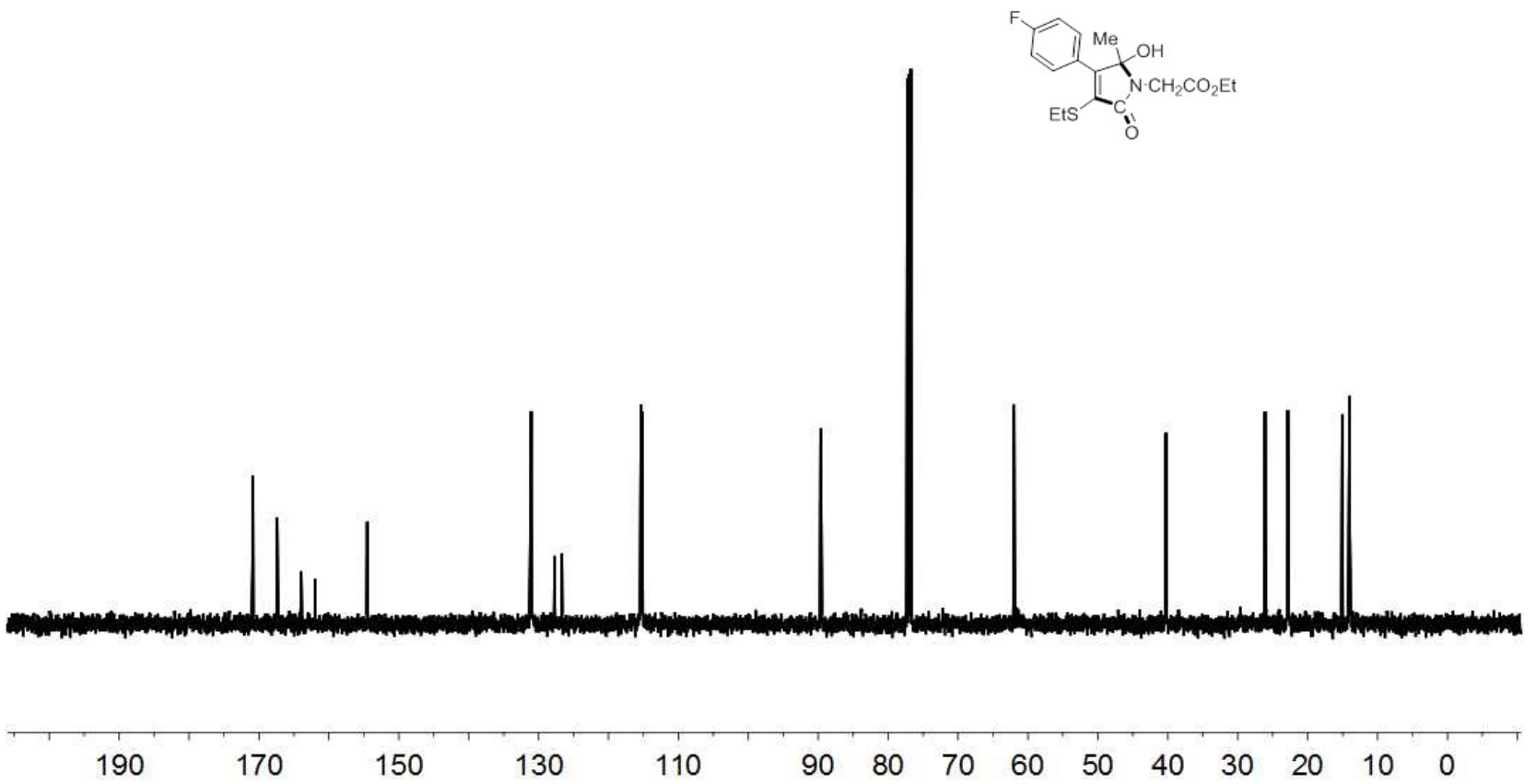



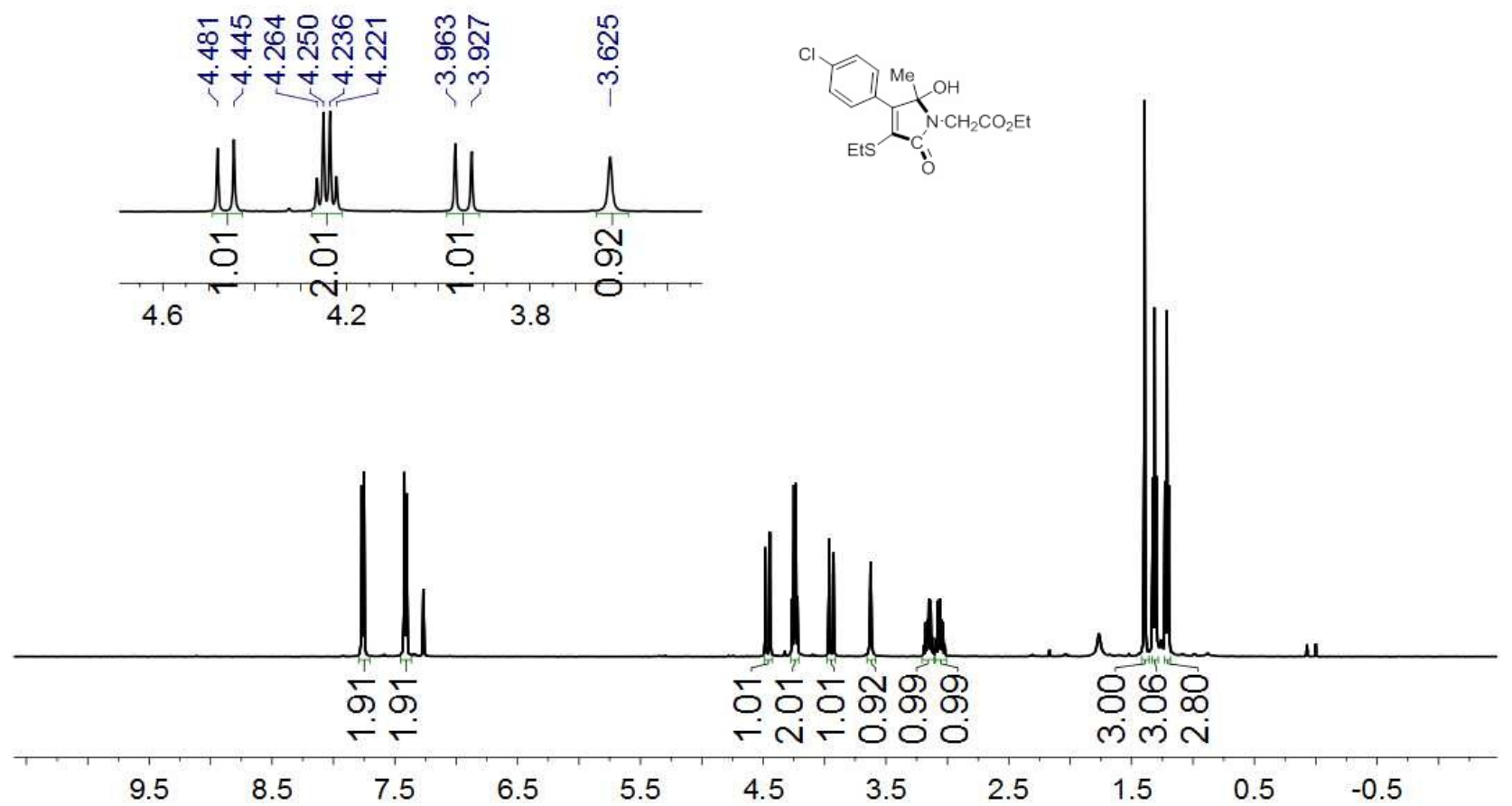

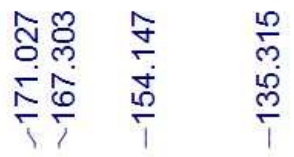

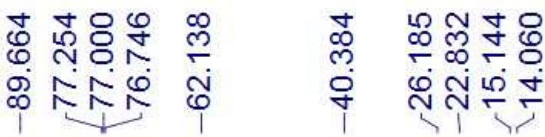
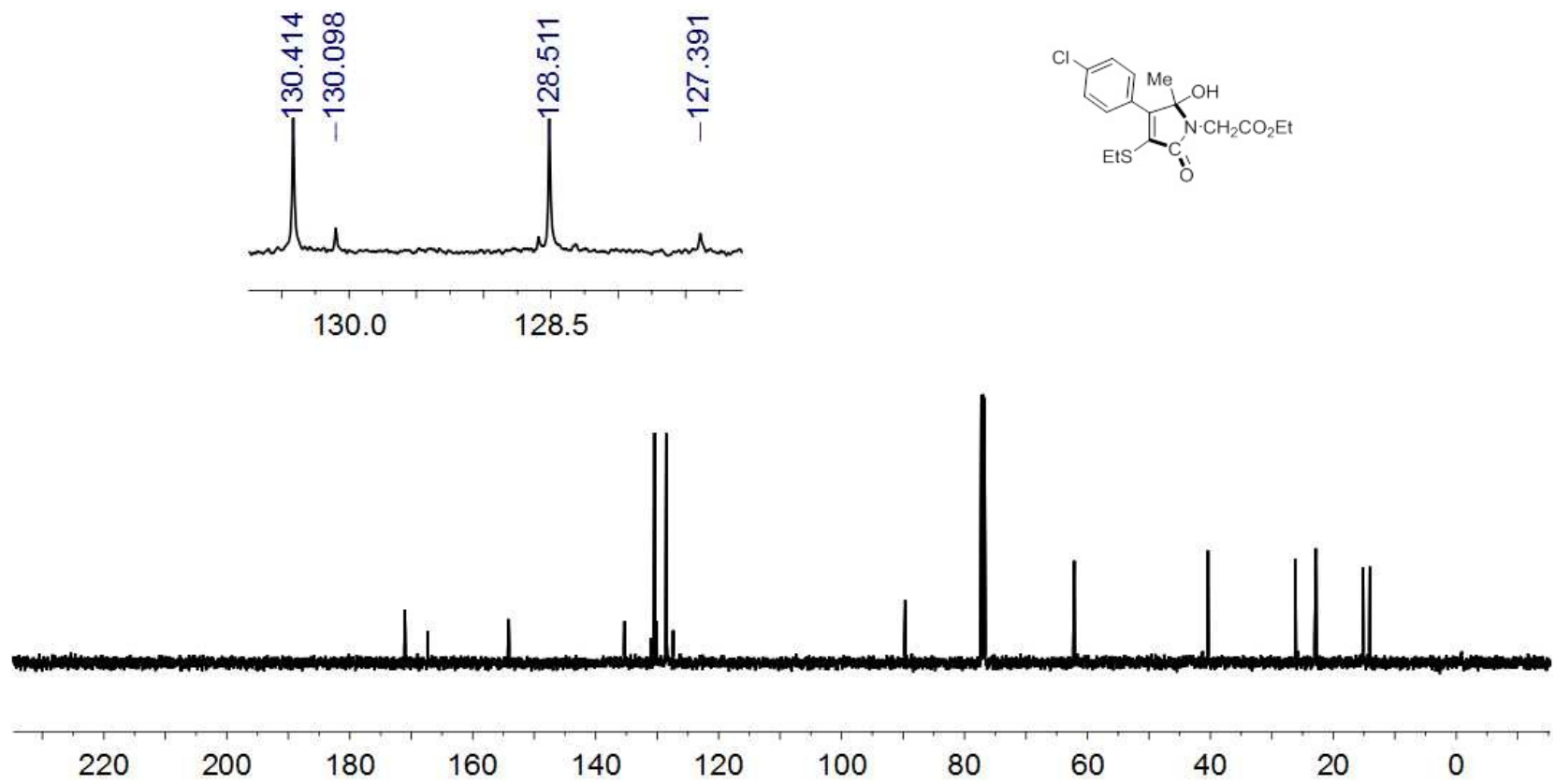


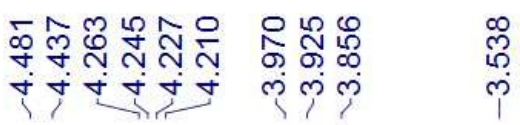
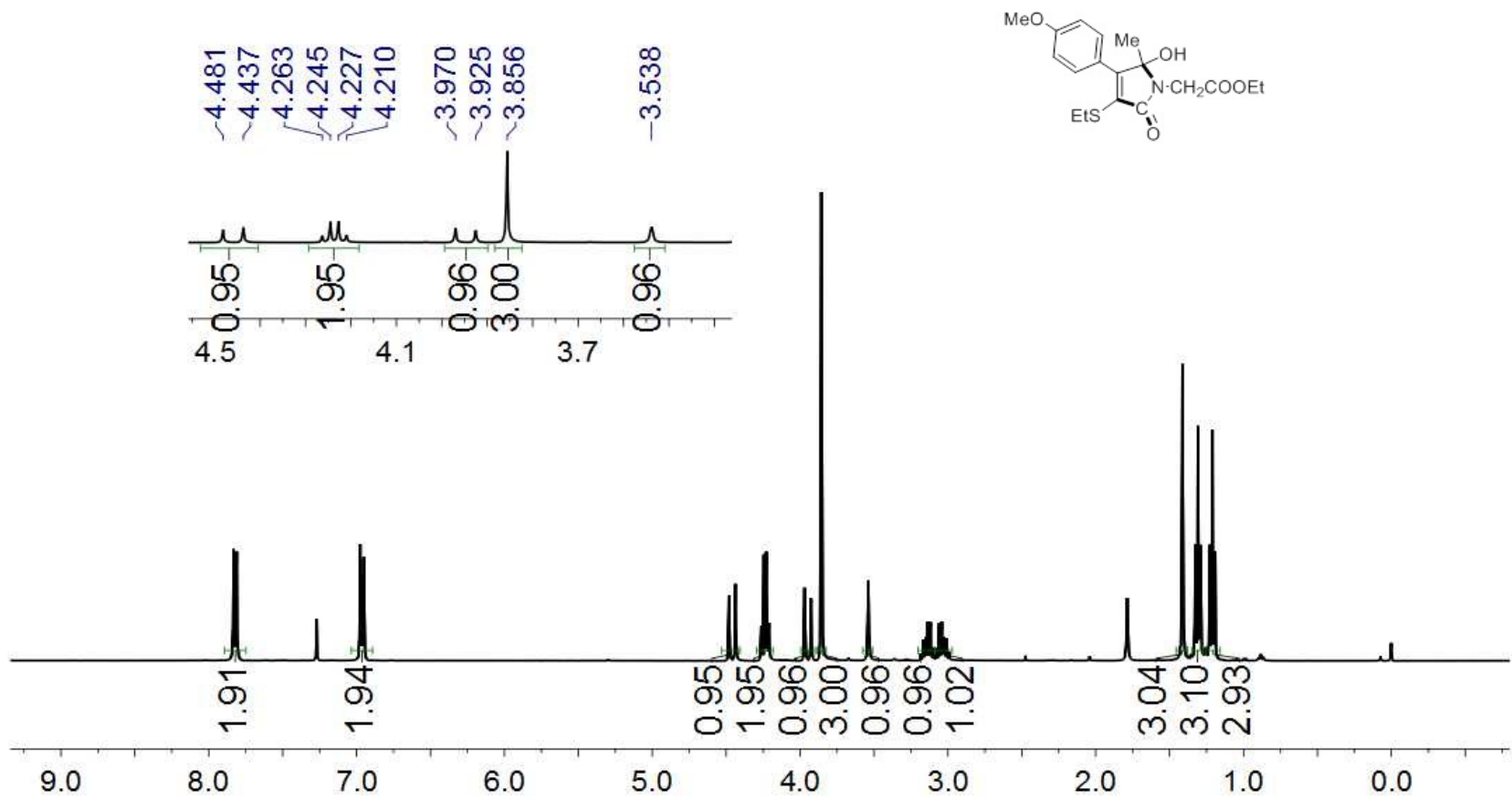

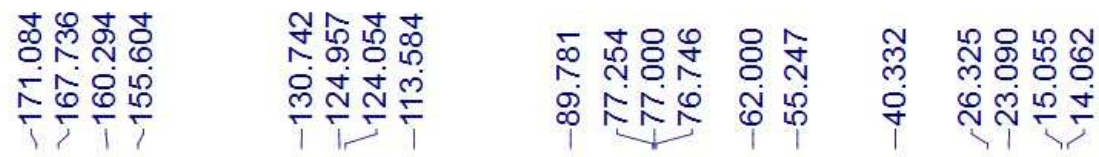

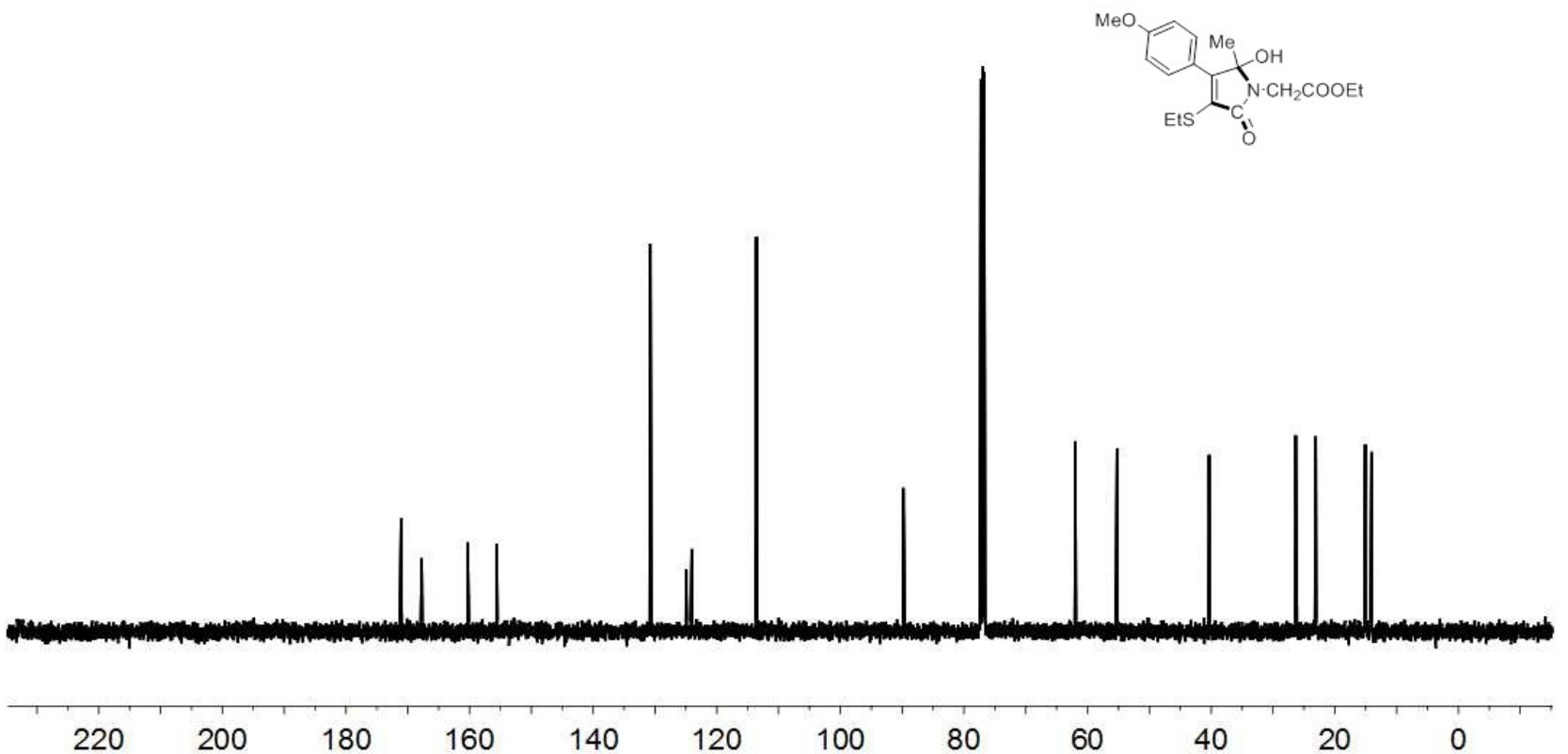



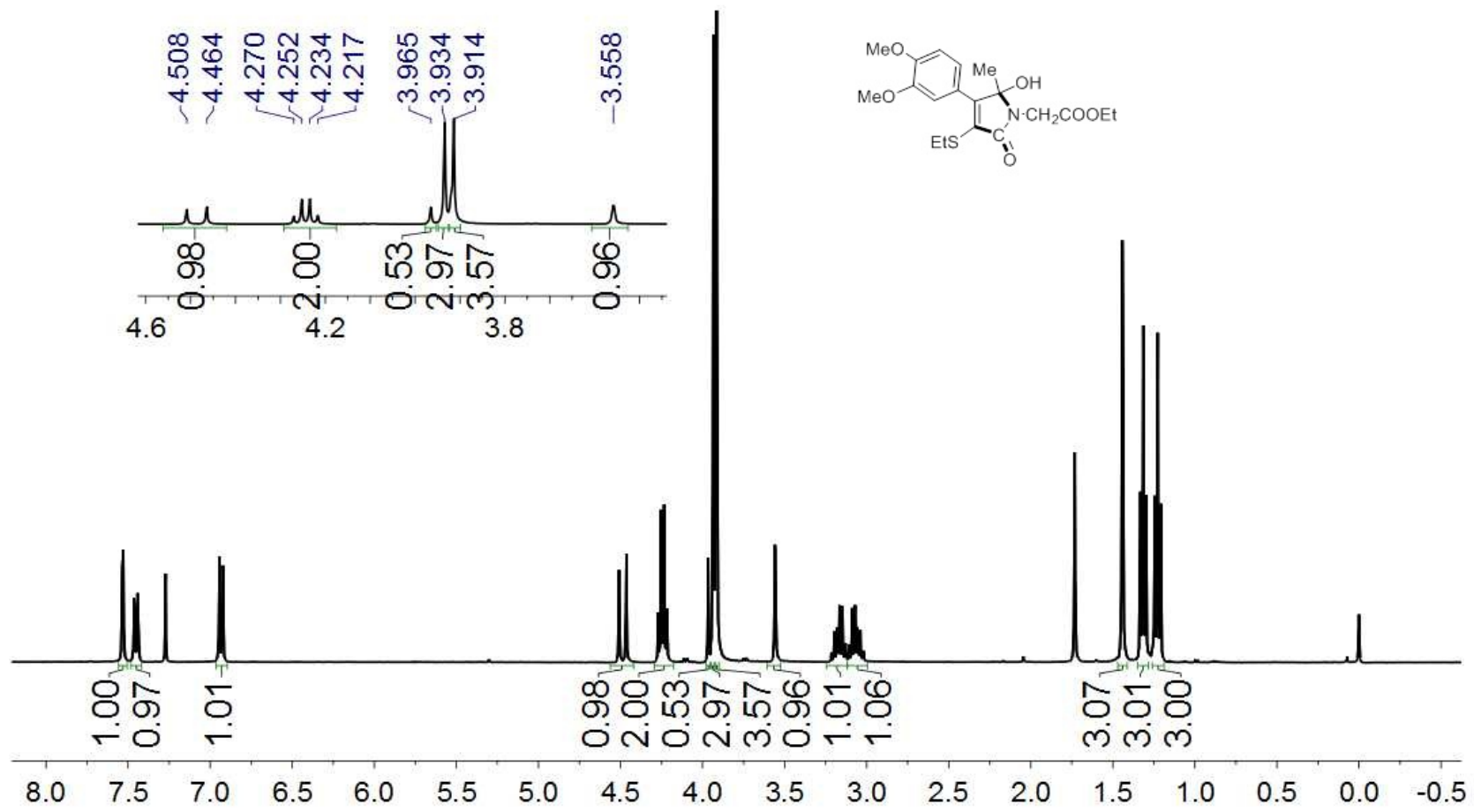

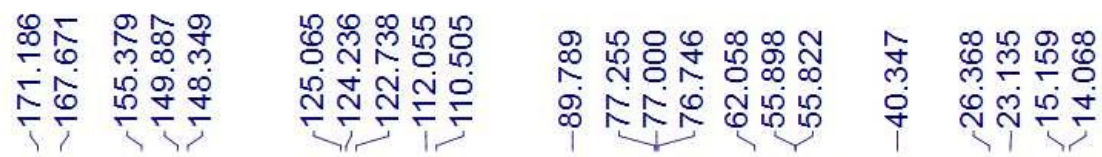

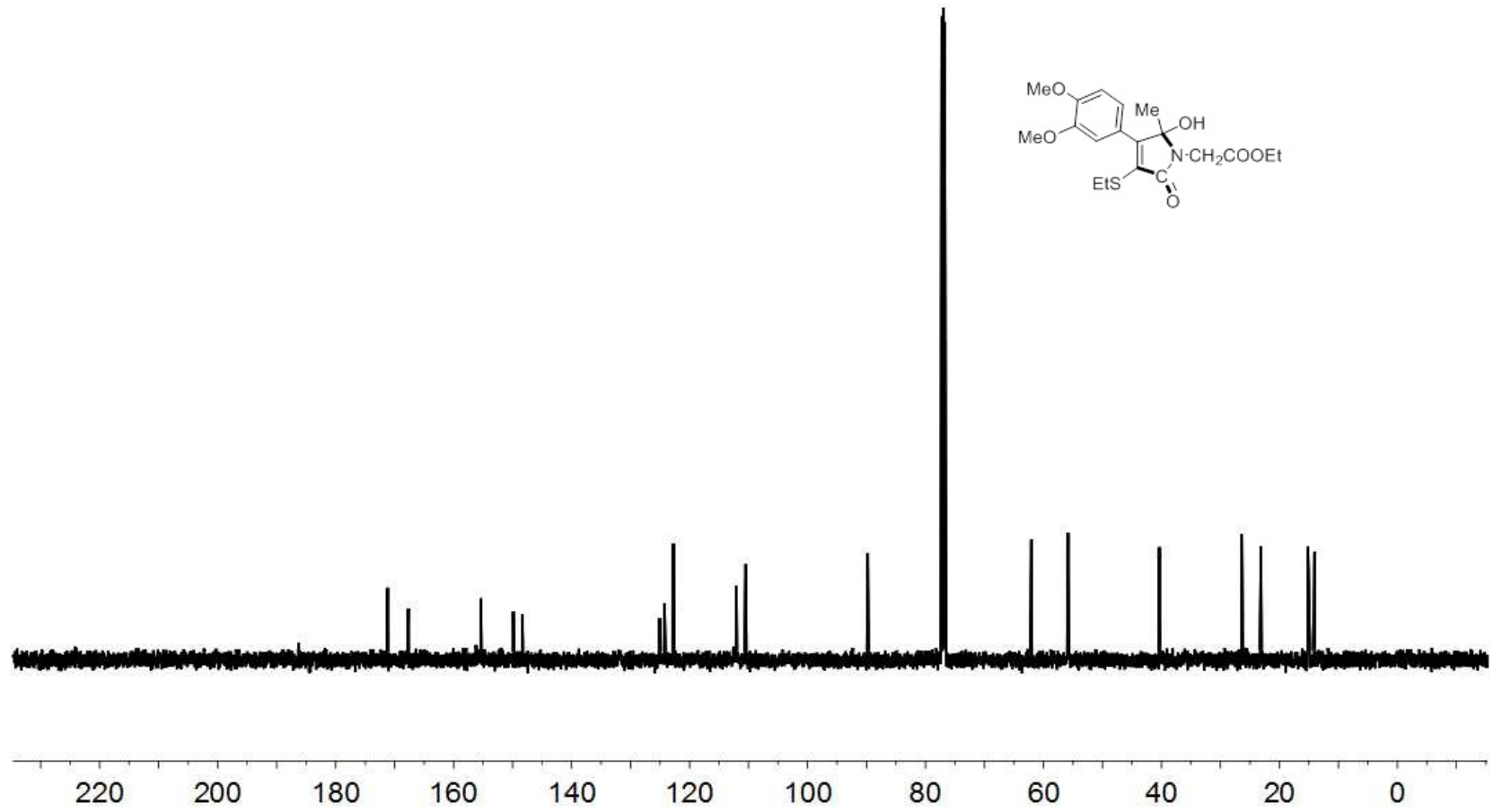


3e

\%

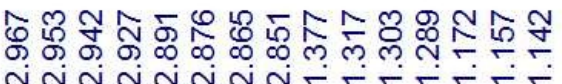

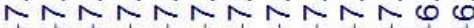
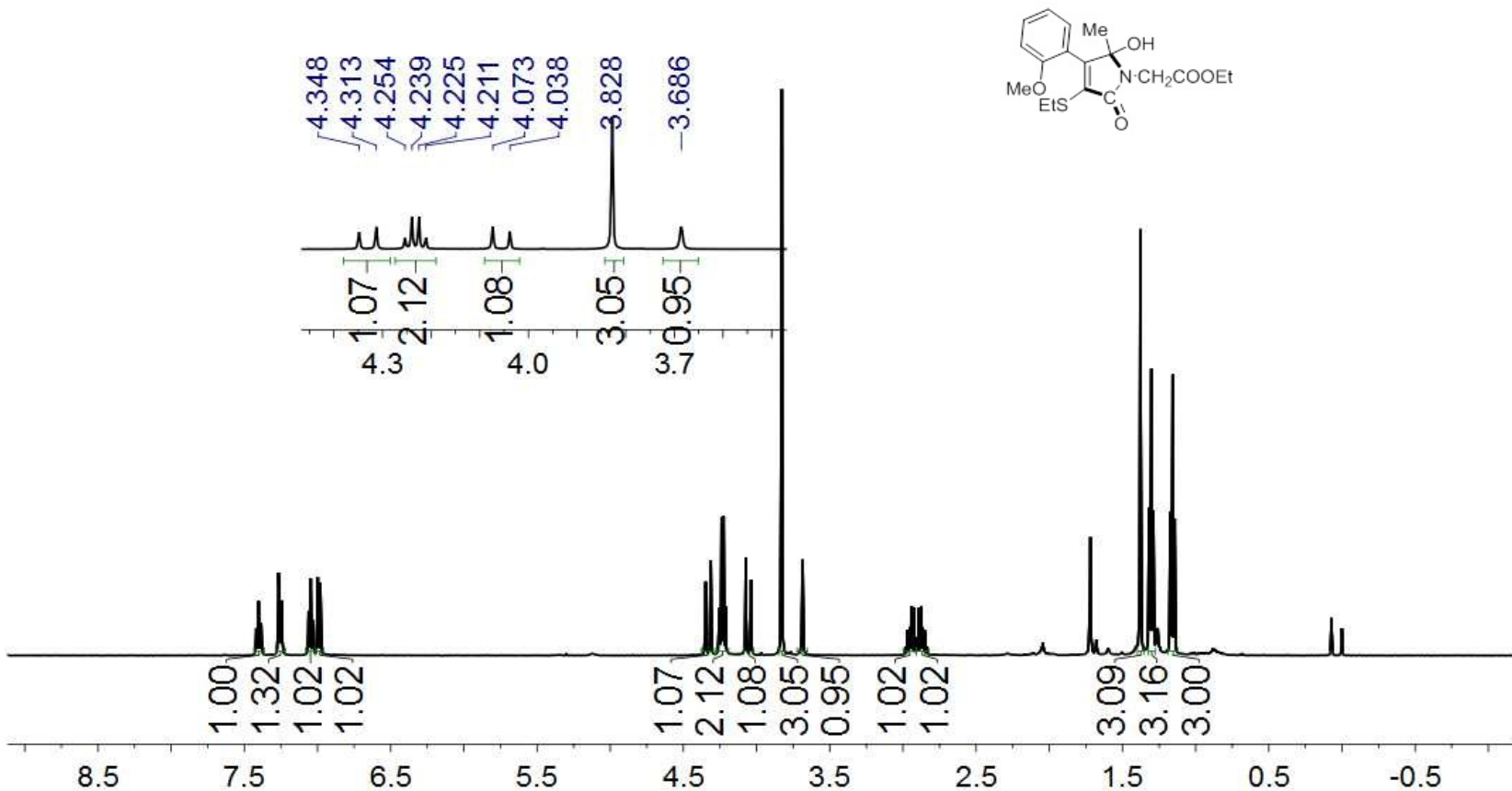

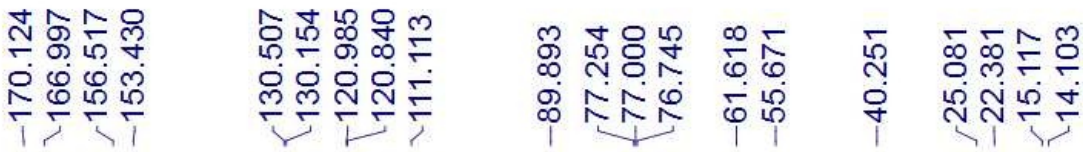
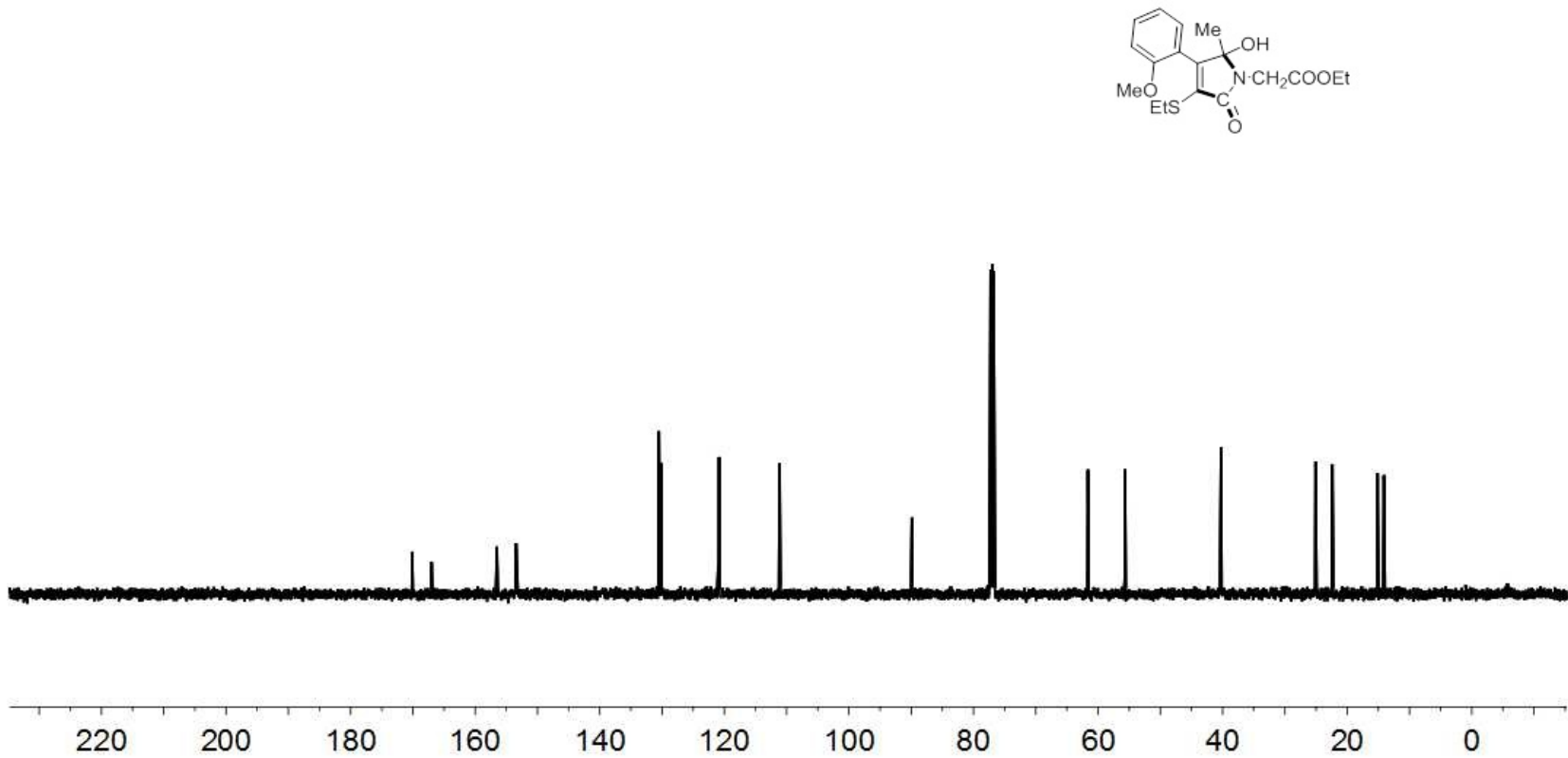

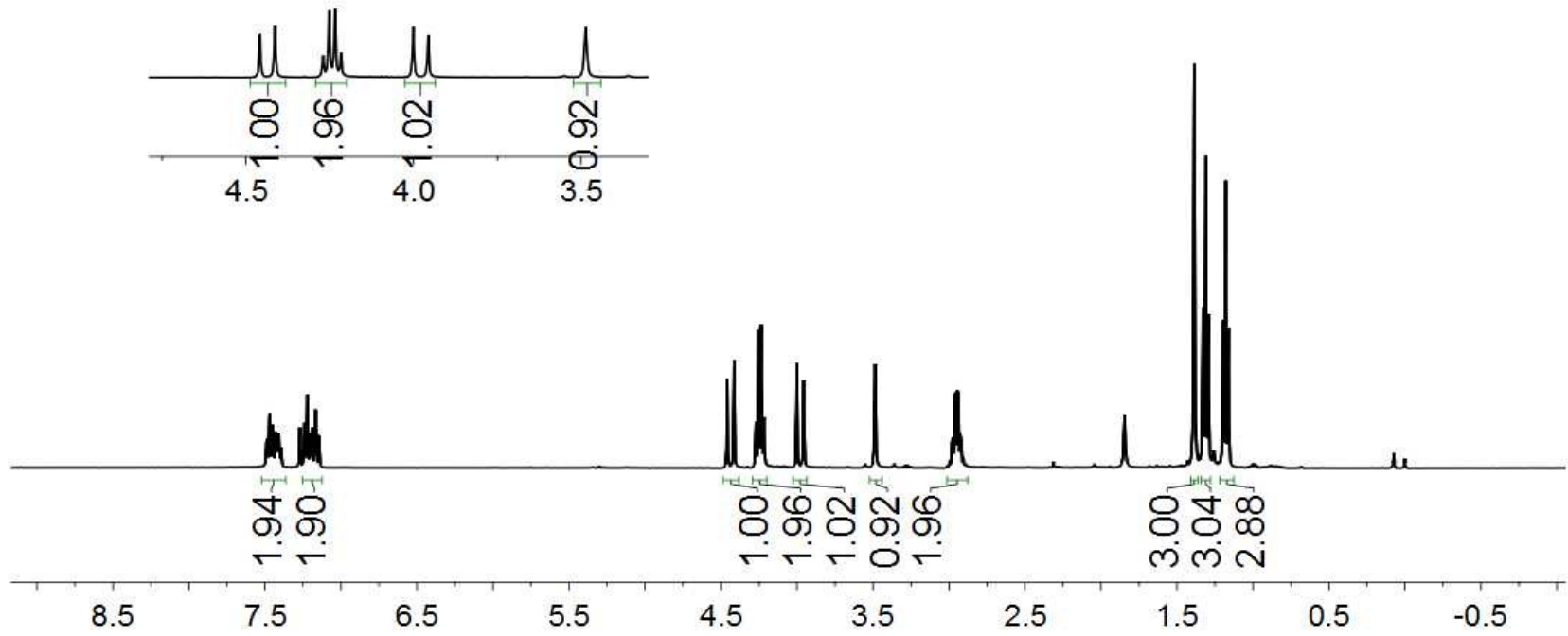

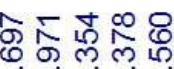

용요

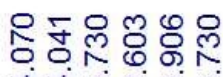

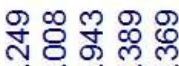

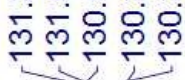
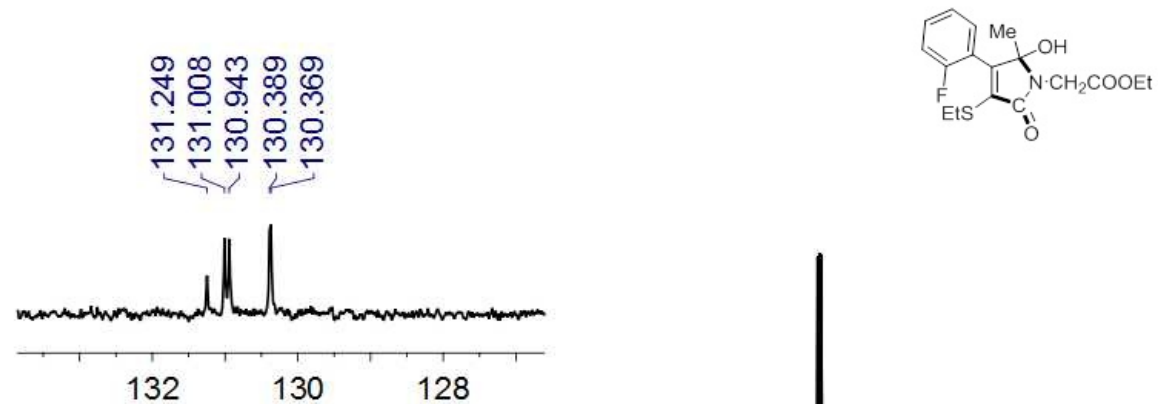


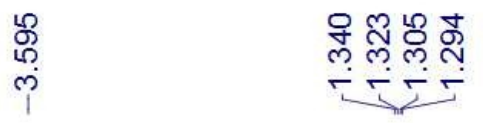

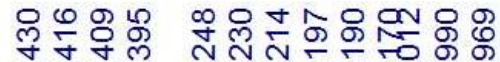

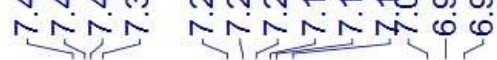
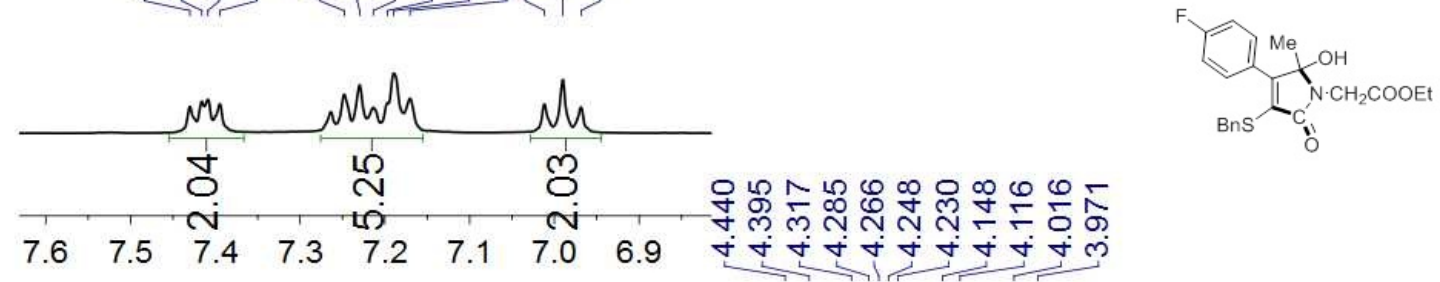

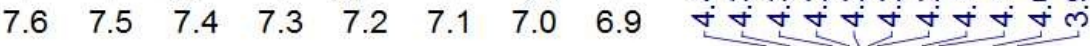
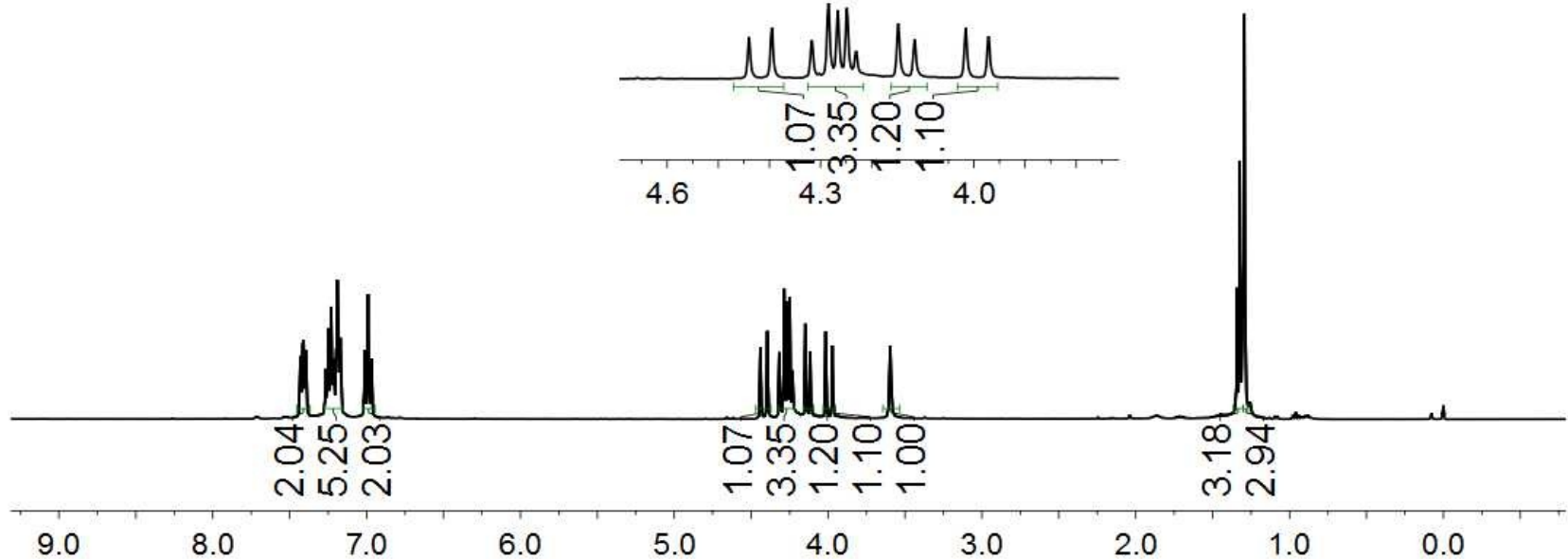

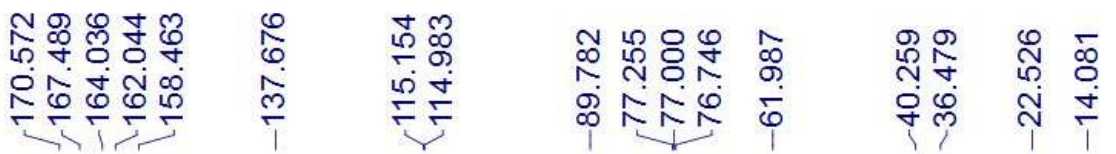

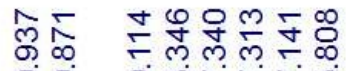

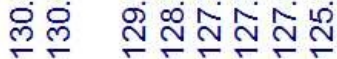
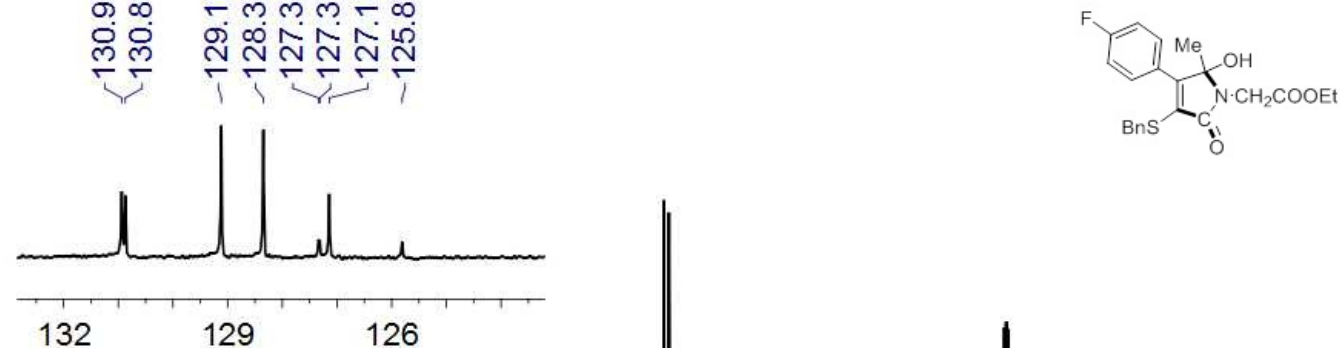

132

129

26

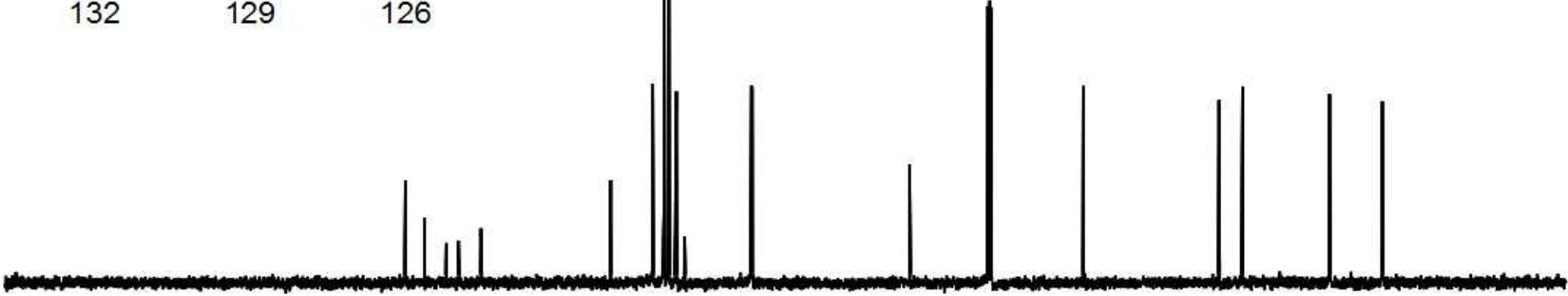

140

120

100

80

60

40

20 

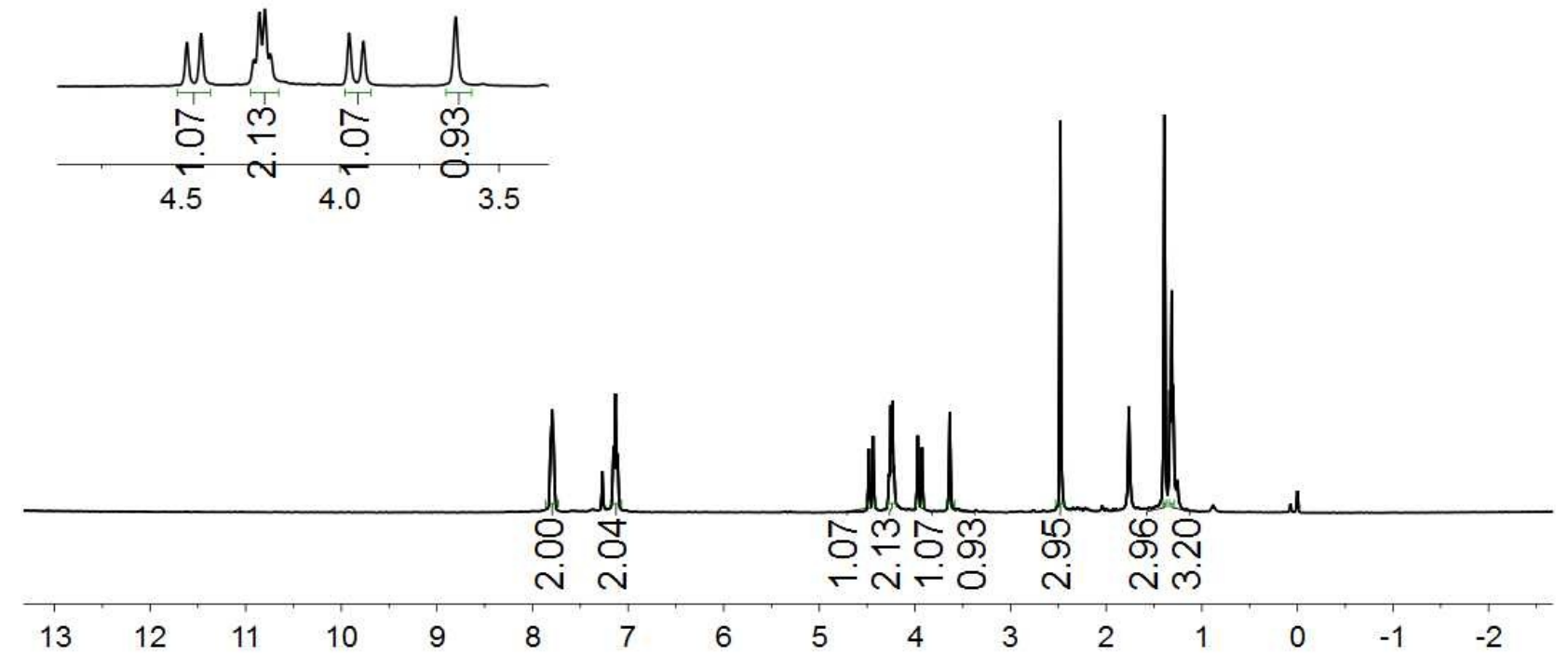

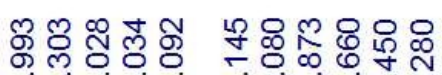

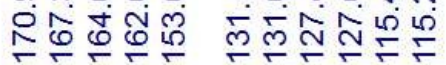

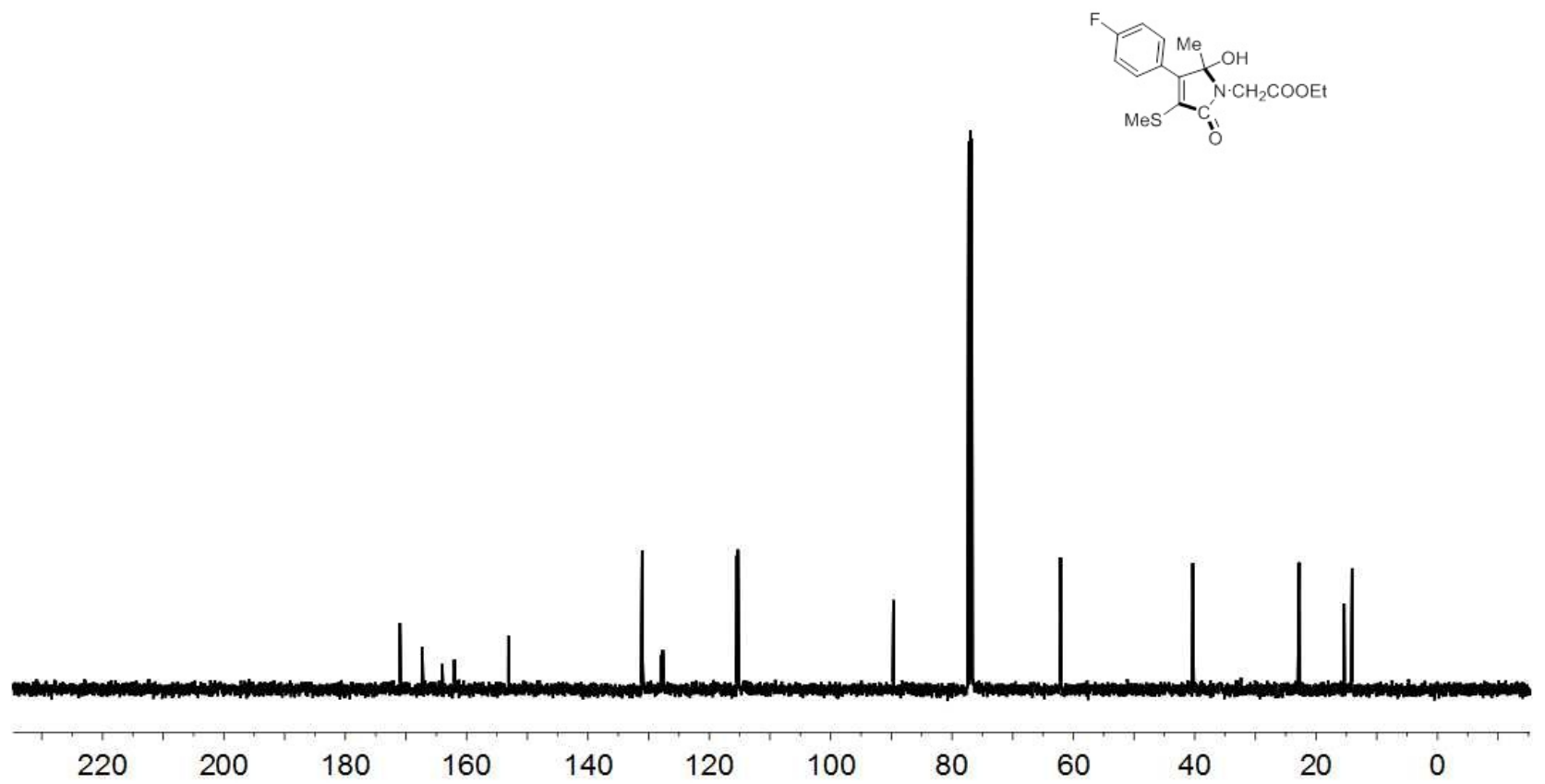



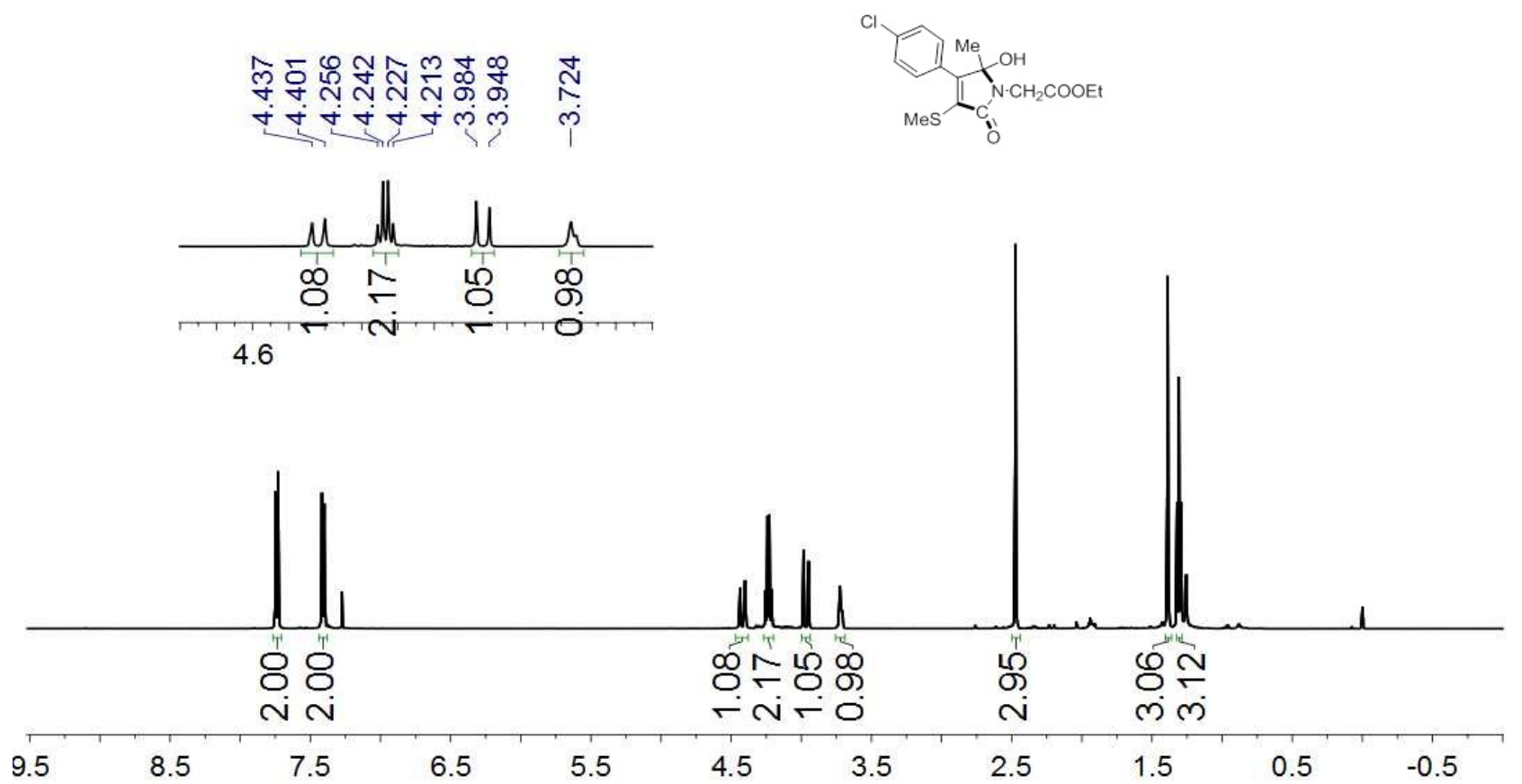

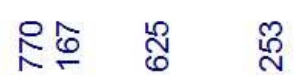

눈

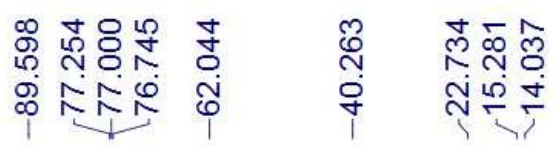

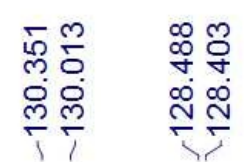
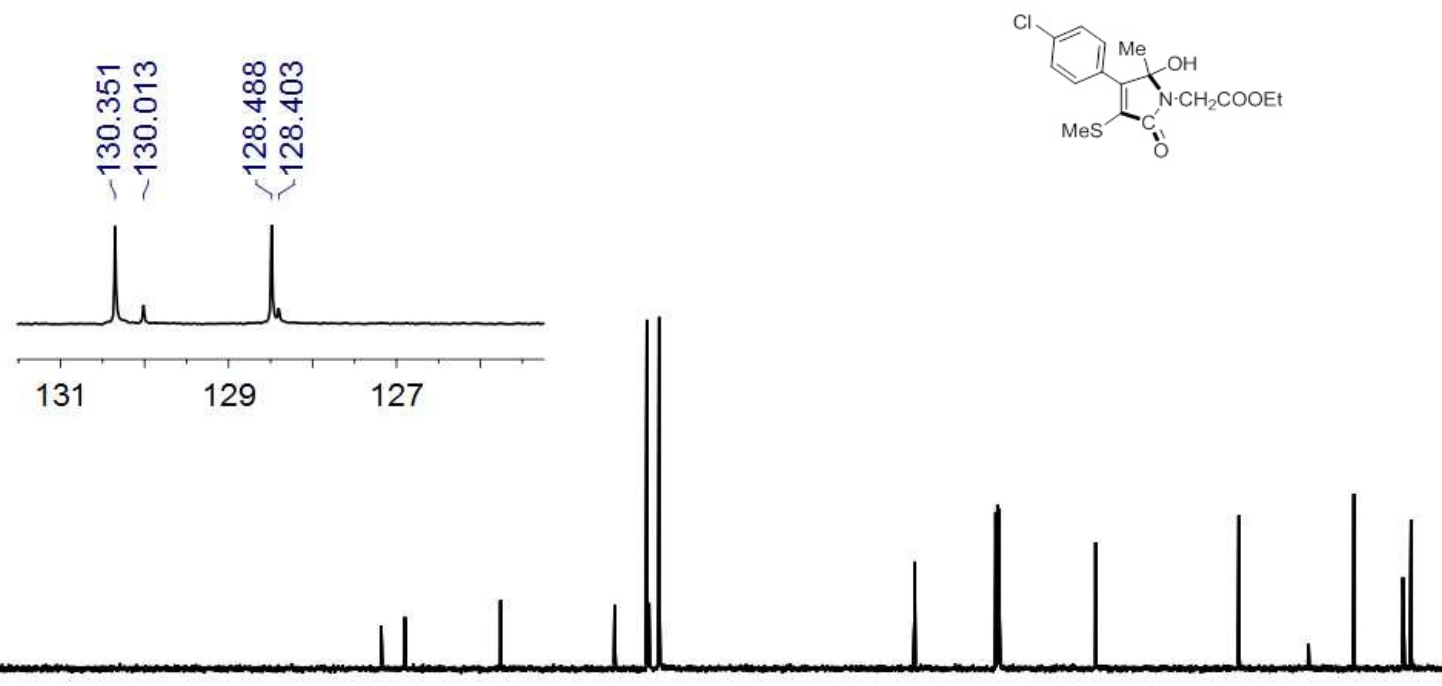

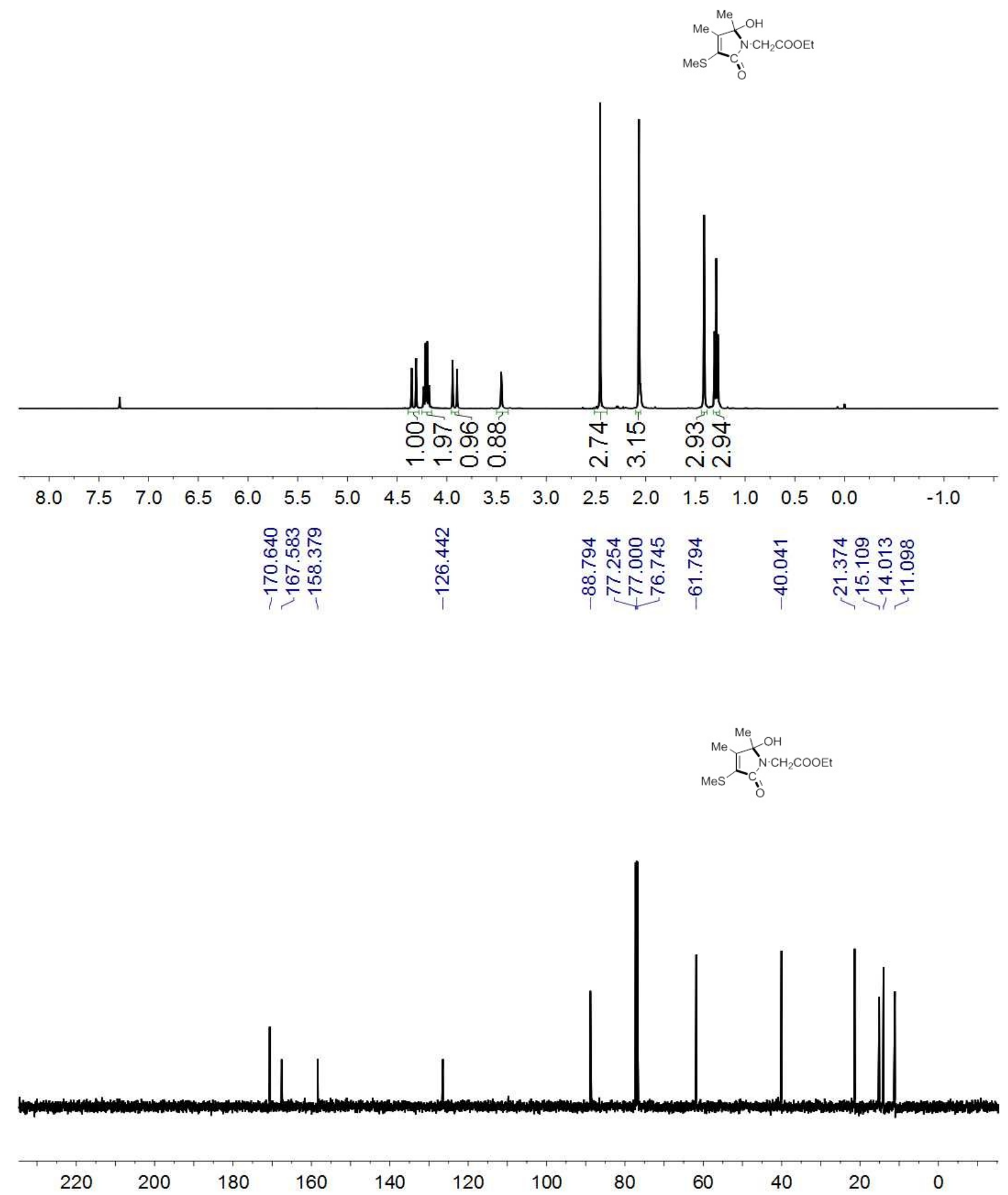


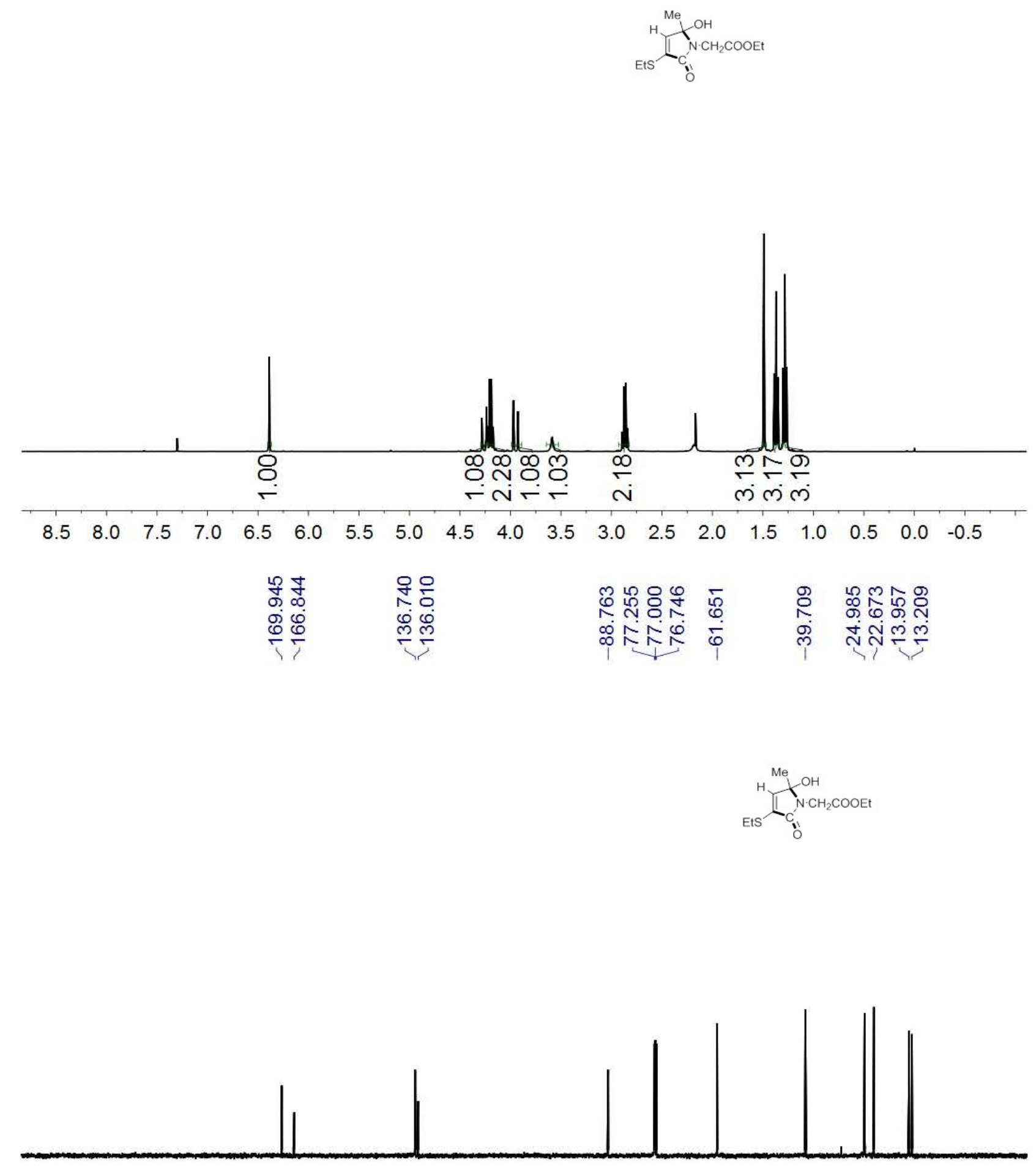

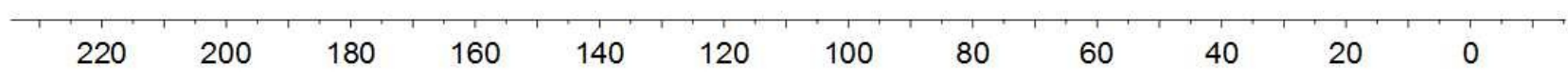



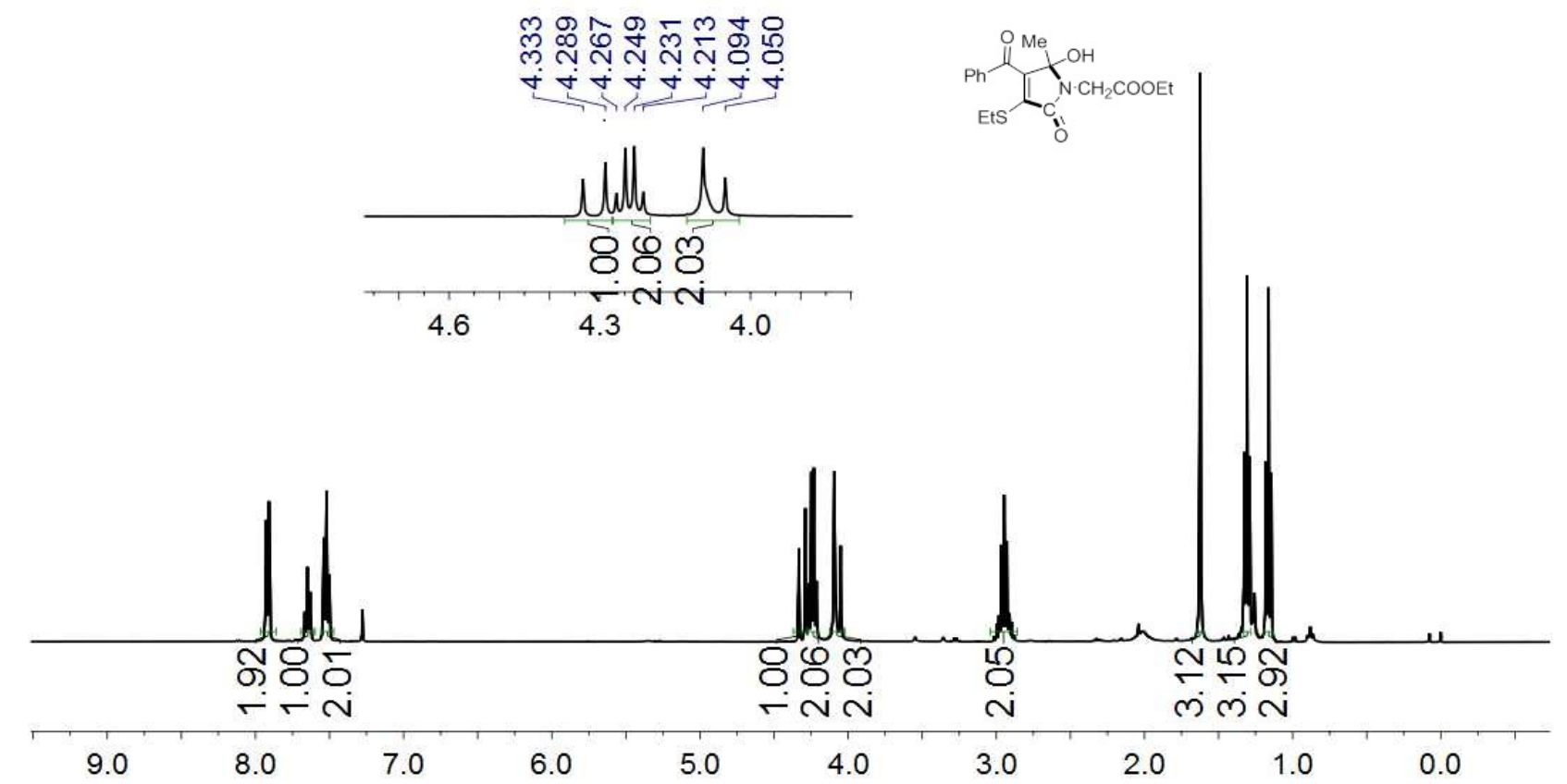

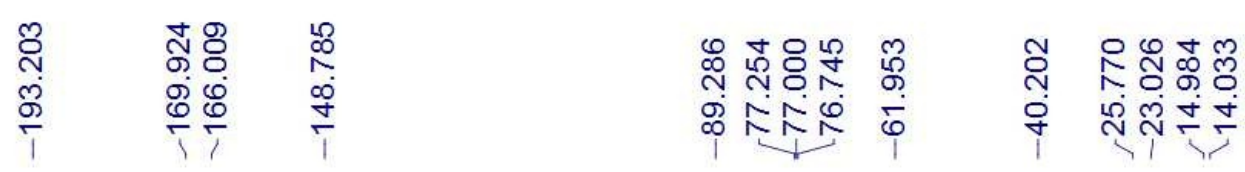

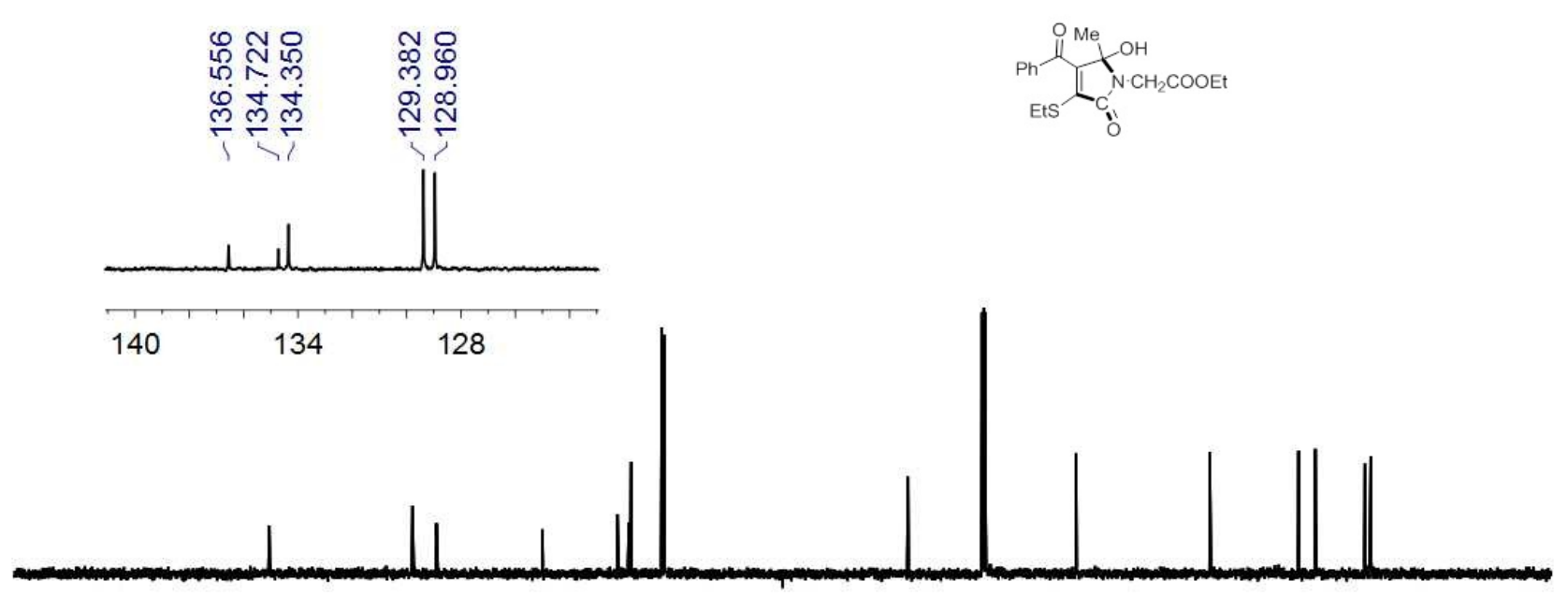

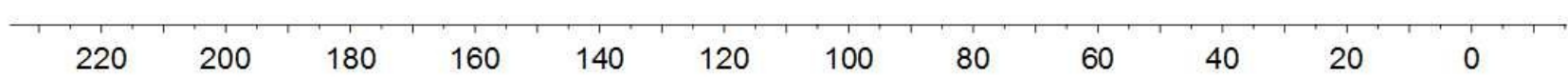




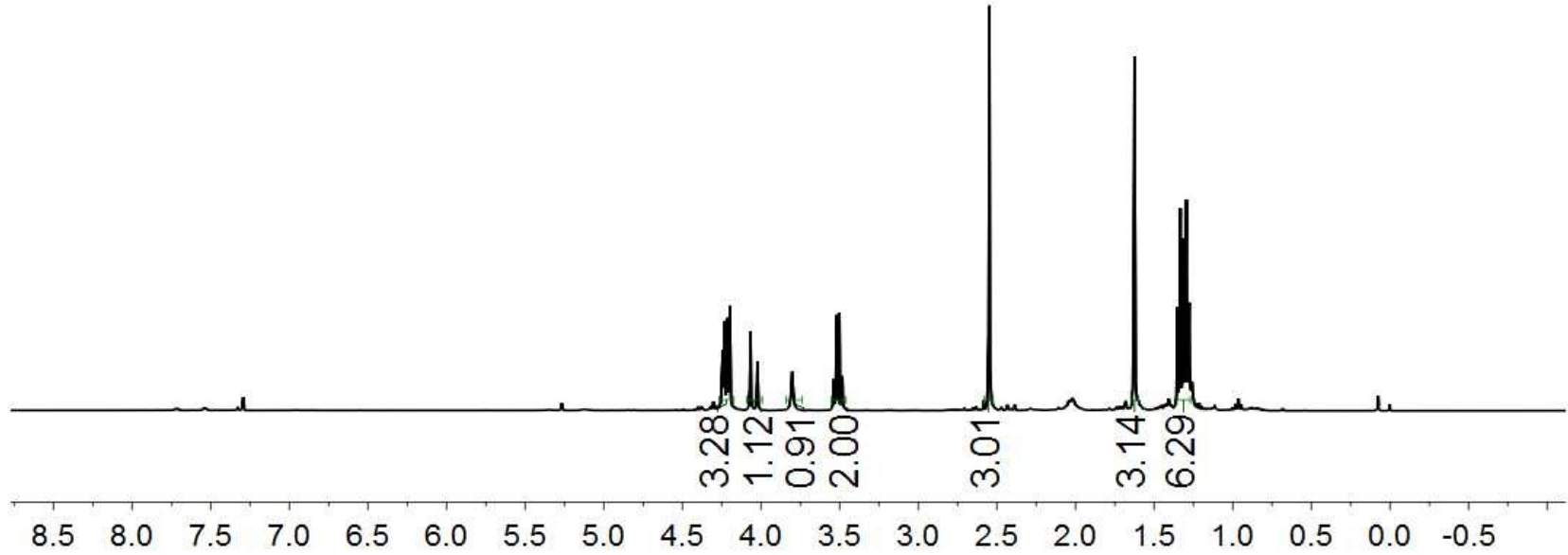

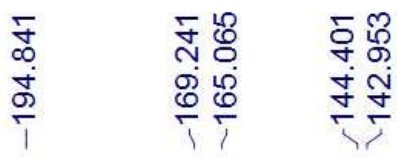

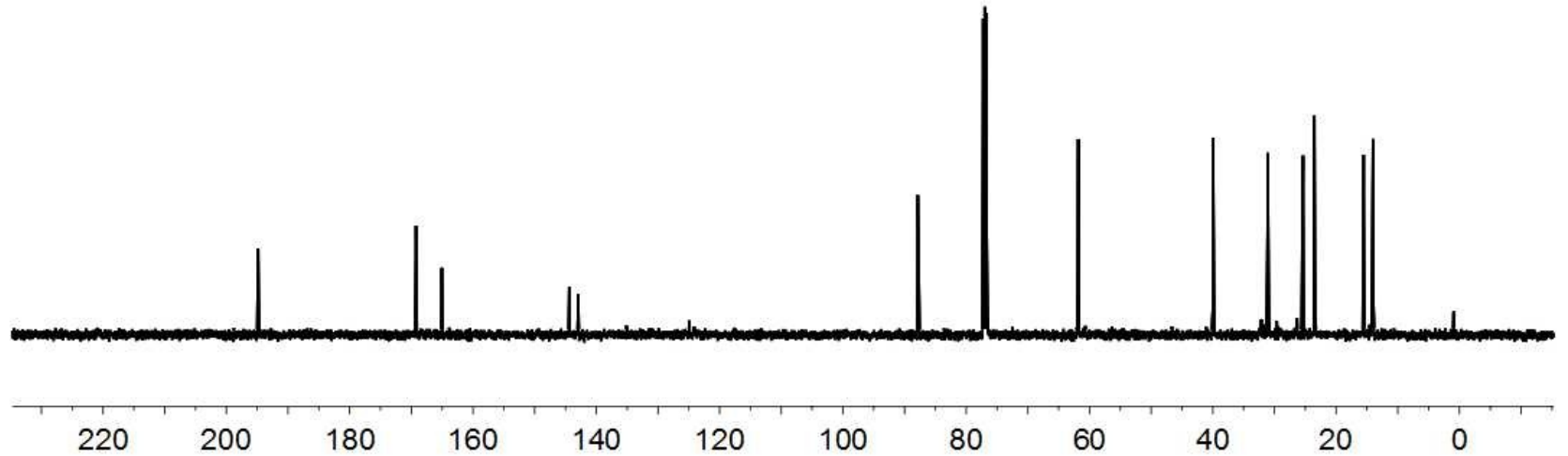



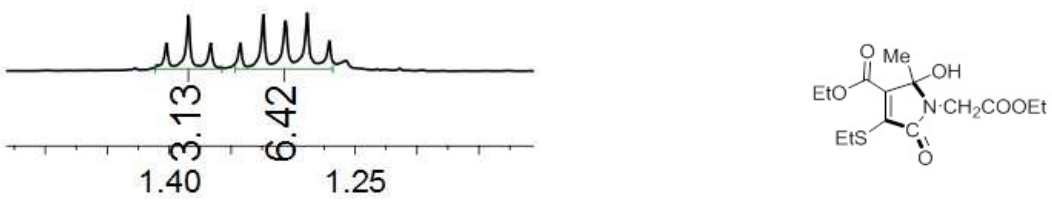

œ

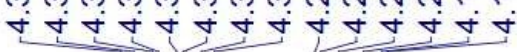

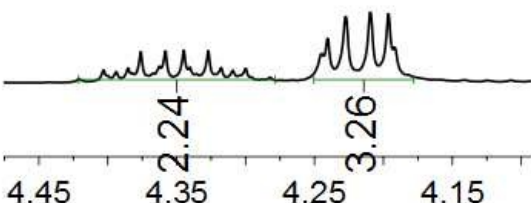

\section{$+0 \sim 2$}

ํำ

तें -

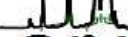

$8 \cong \mathrm{d}$

लं

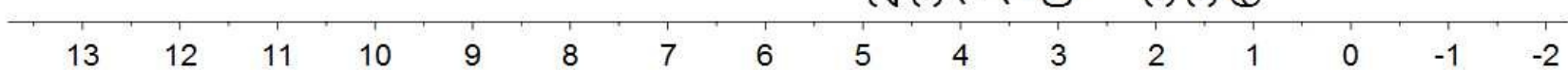

華華苋

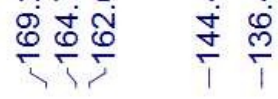

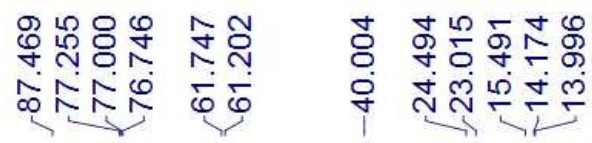

EtS

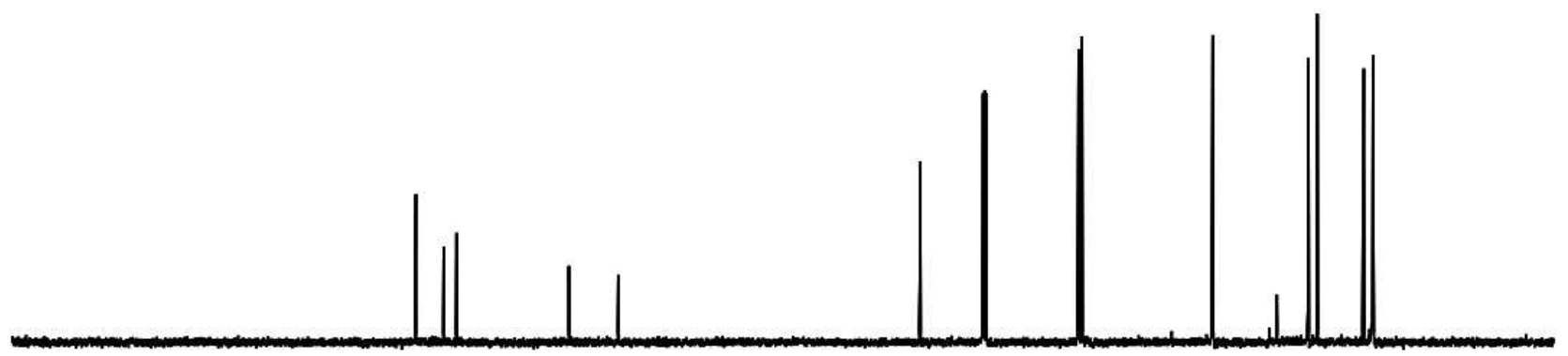

220

200

180

160

140

120

80

60

40

20 


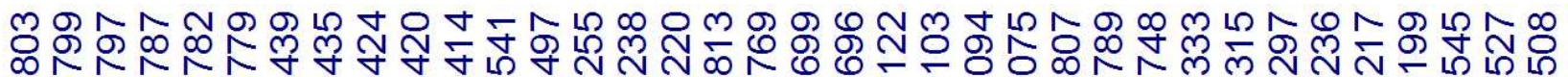

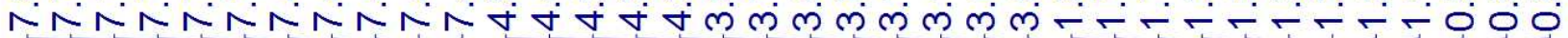

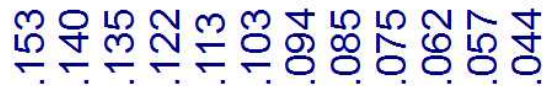

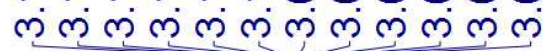
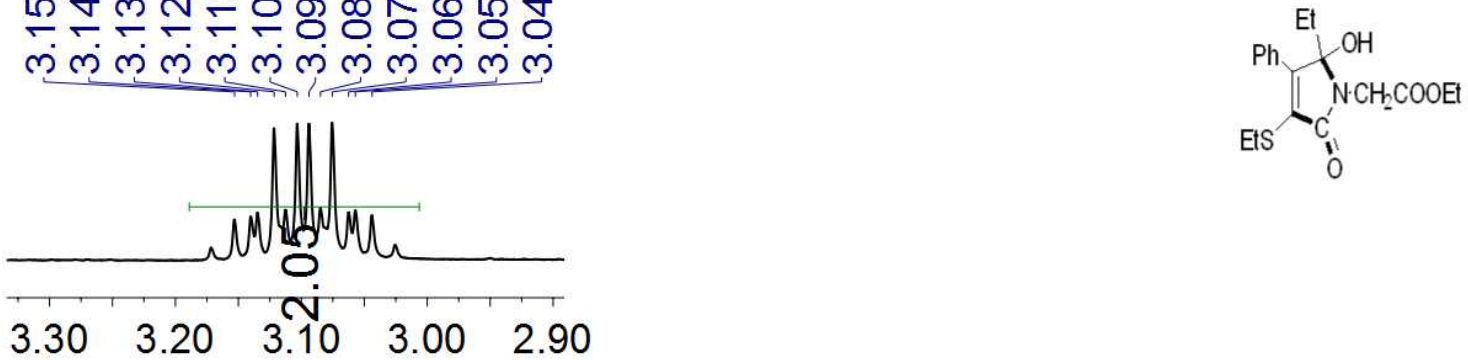

$\begin{array}{lllll}3.30 & 3.20 & 3.10 & 3.00 & 2.90\end{array}$

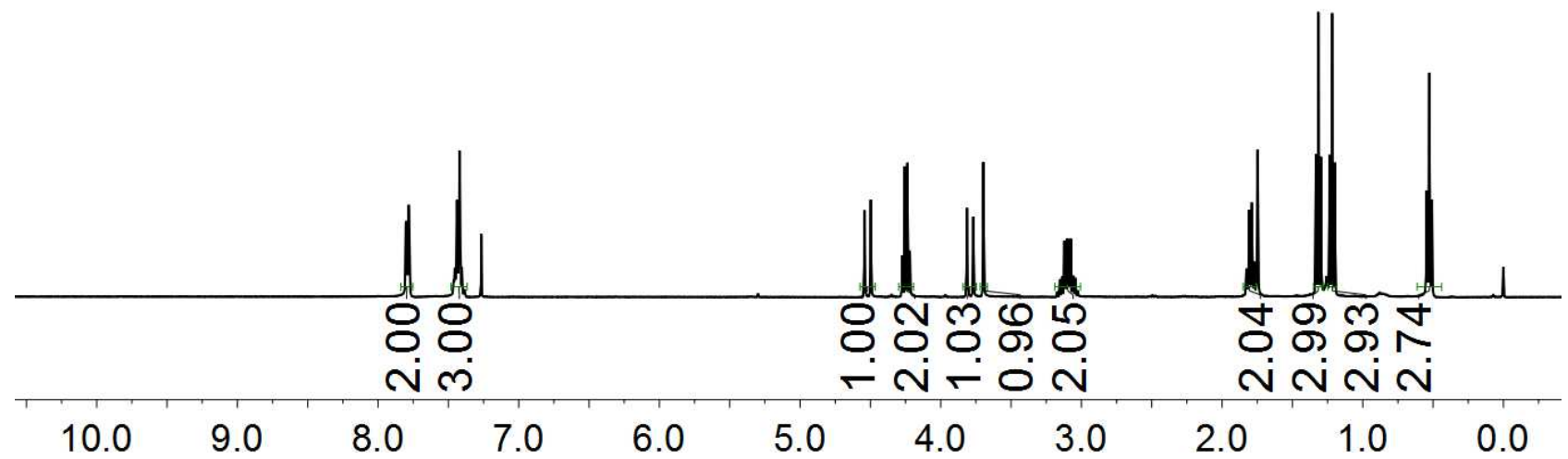

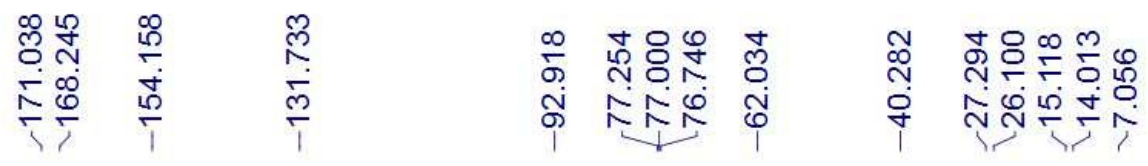

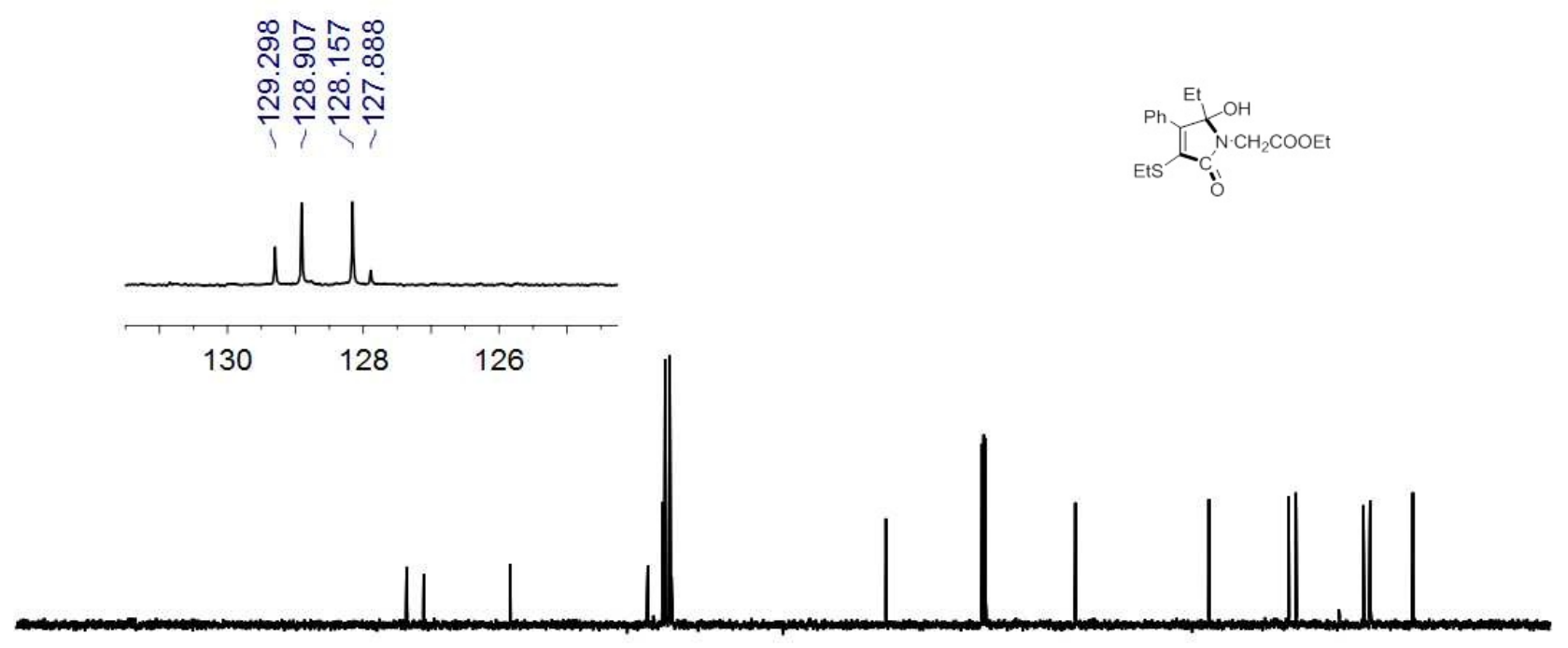




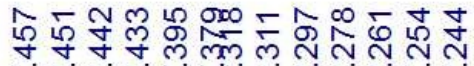

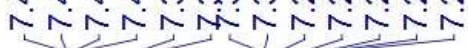
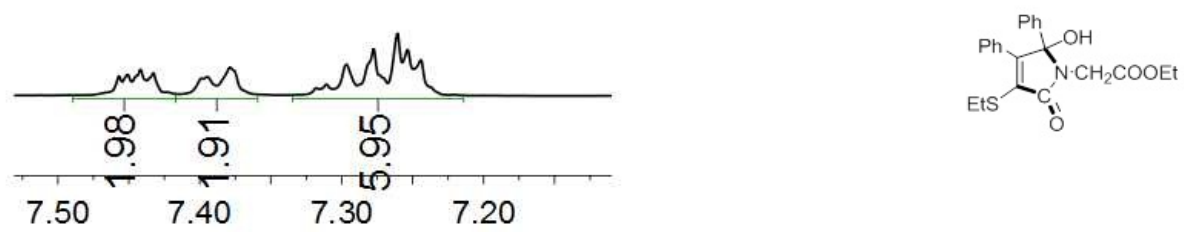

$\begin{array}{llll}7.50 & 7.40 & 7.30 & 7.20\end{array}$
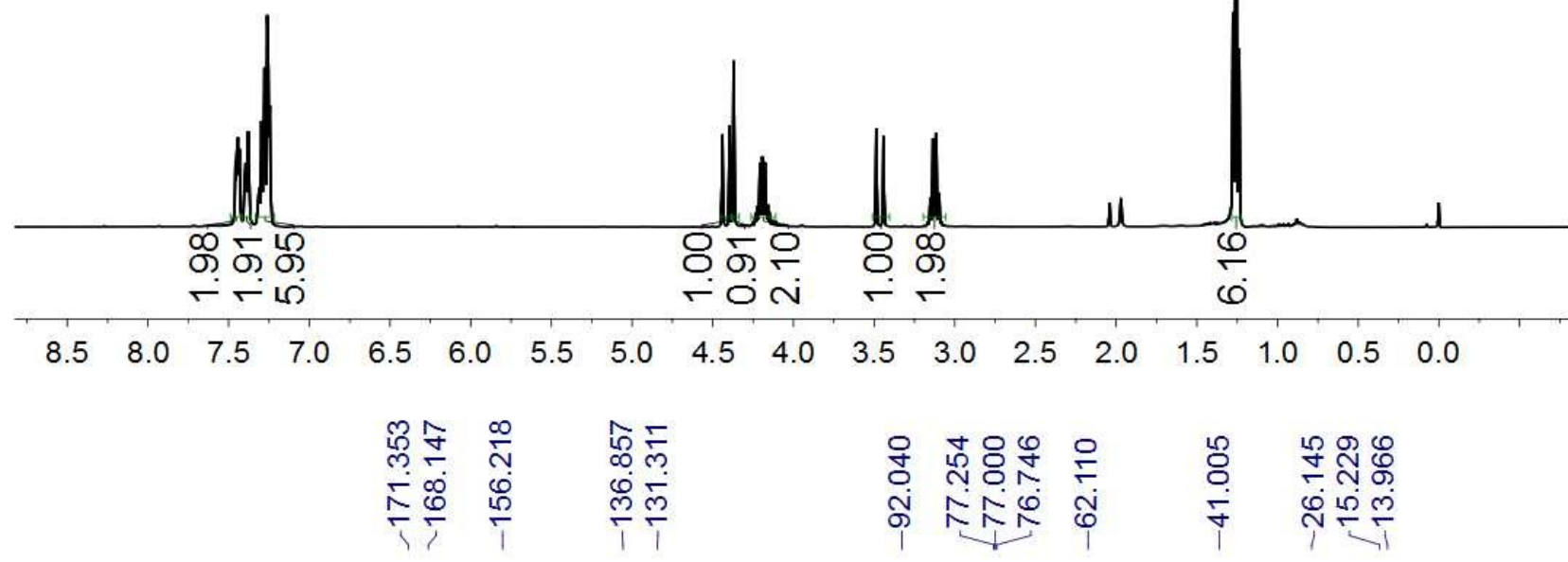

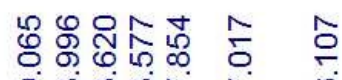

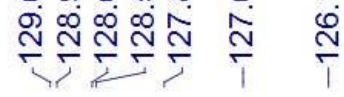

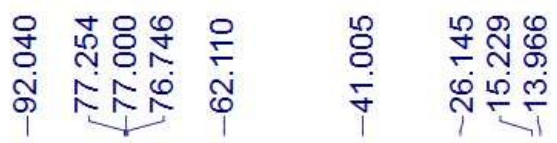
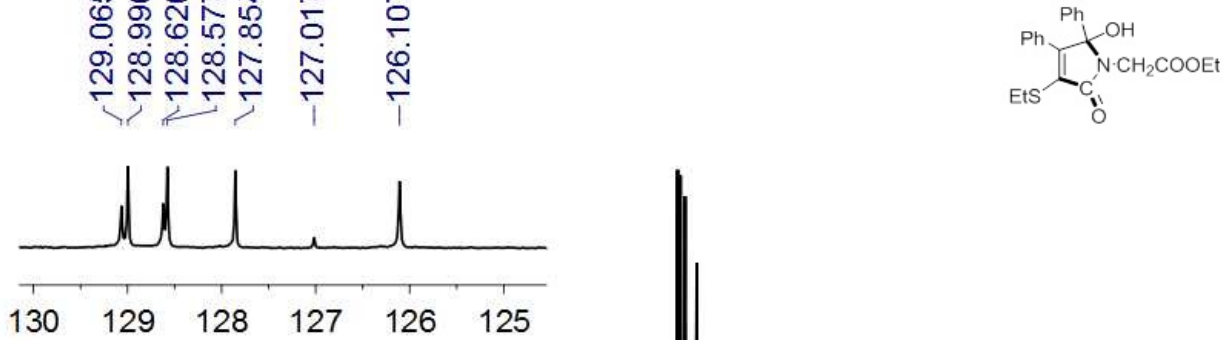

$\begin{array}{llllll}130 & 129 & 128 & 127 & 126 & 125\end{array}$

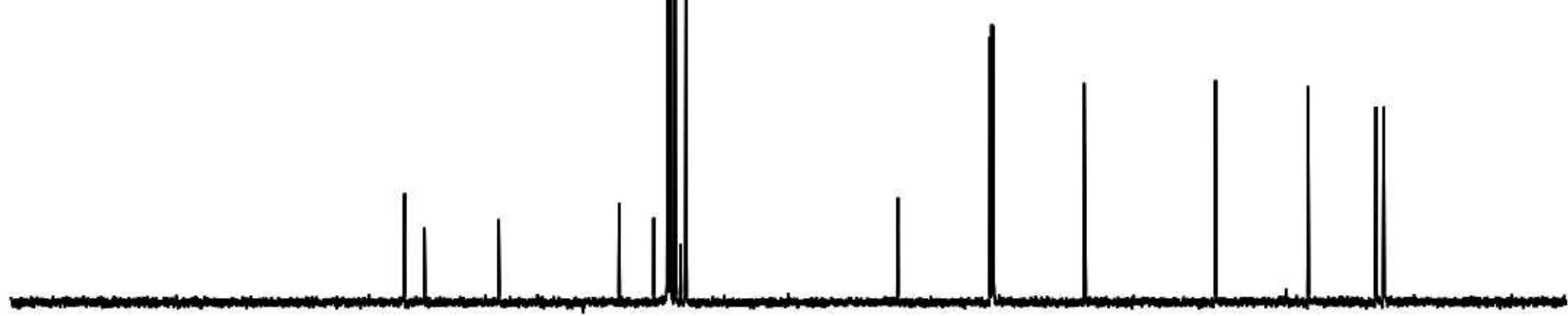

220

200

180

160

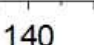

120

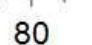

60

40

20 
$3 q$

ॠ

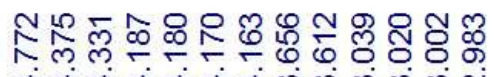

Nin

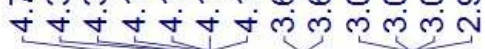

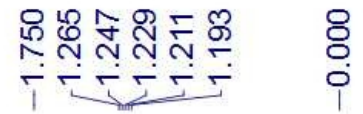
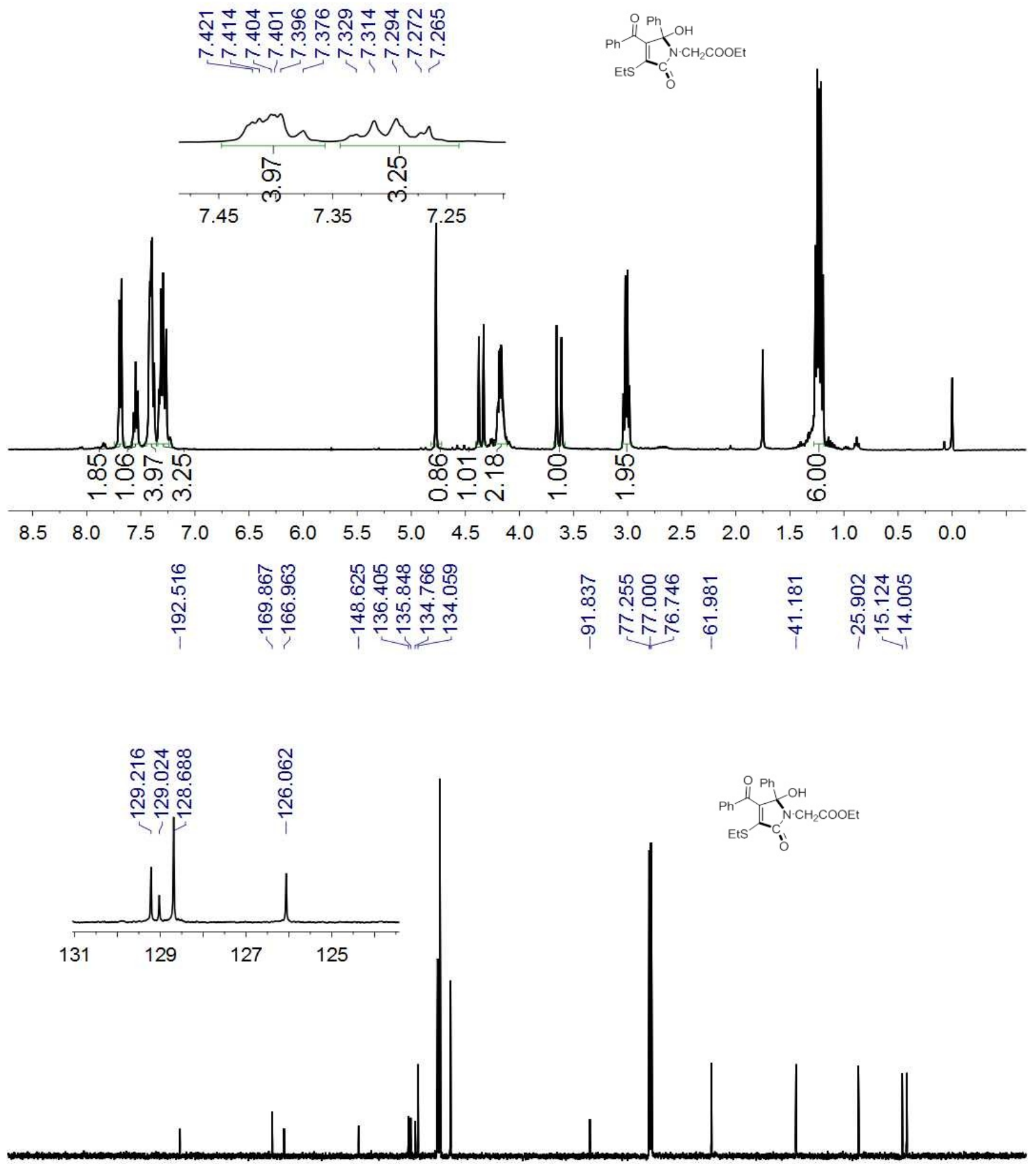

220

200

180

160

140

120

100

80

60

40

20 


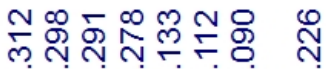

NNNNNA

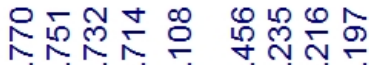

กnก

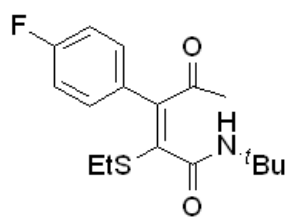

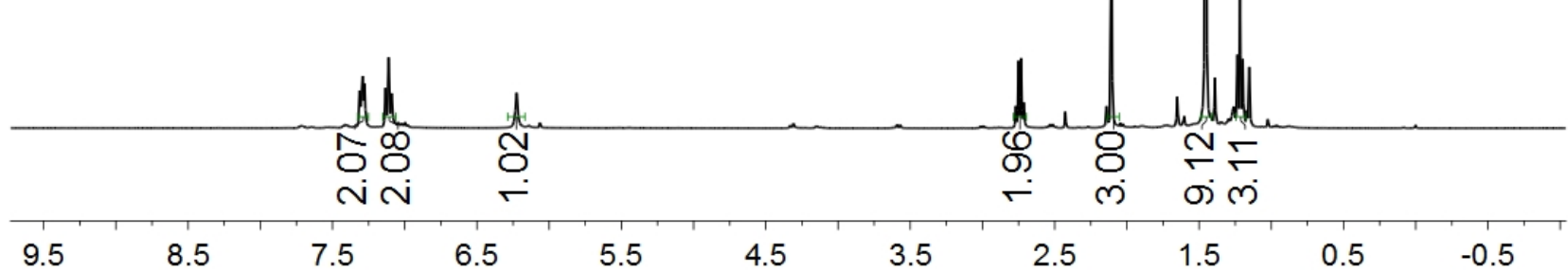

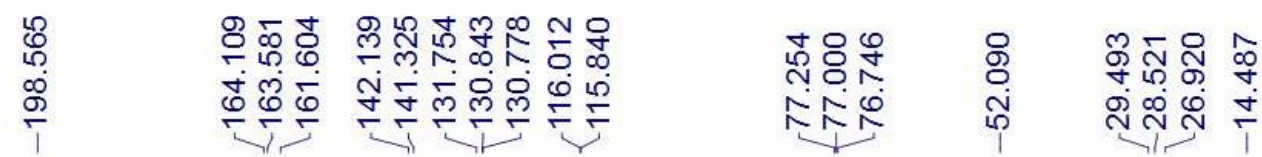

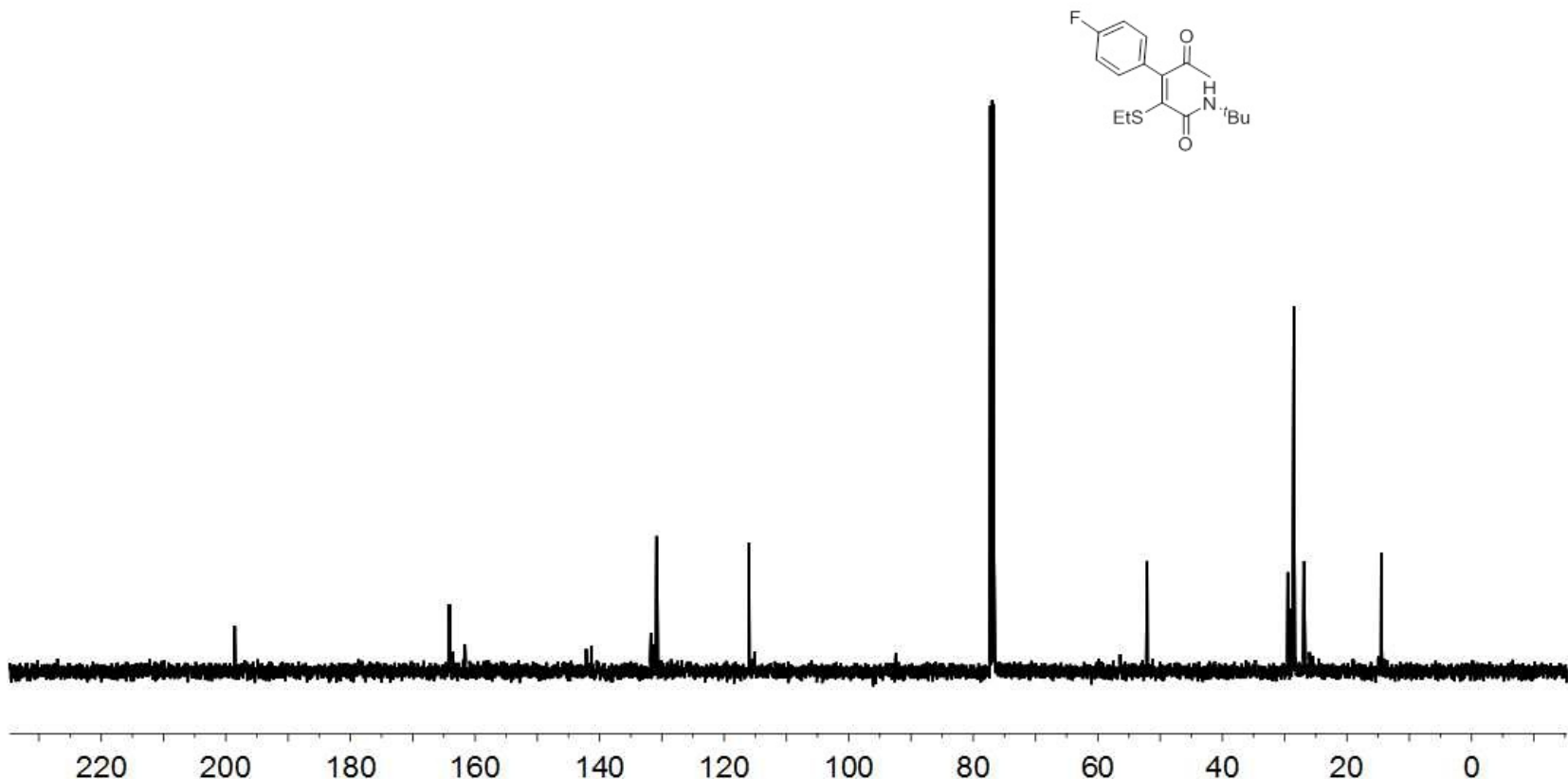


$5 \mathbf{a}$

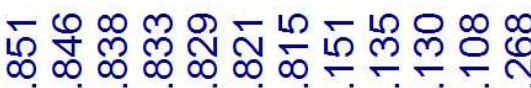

NNNNNNNNNN
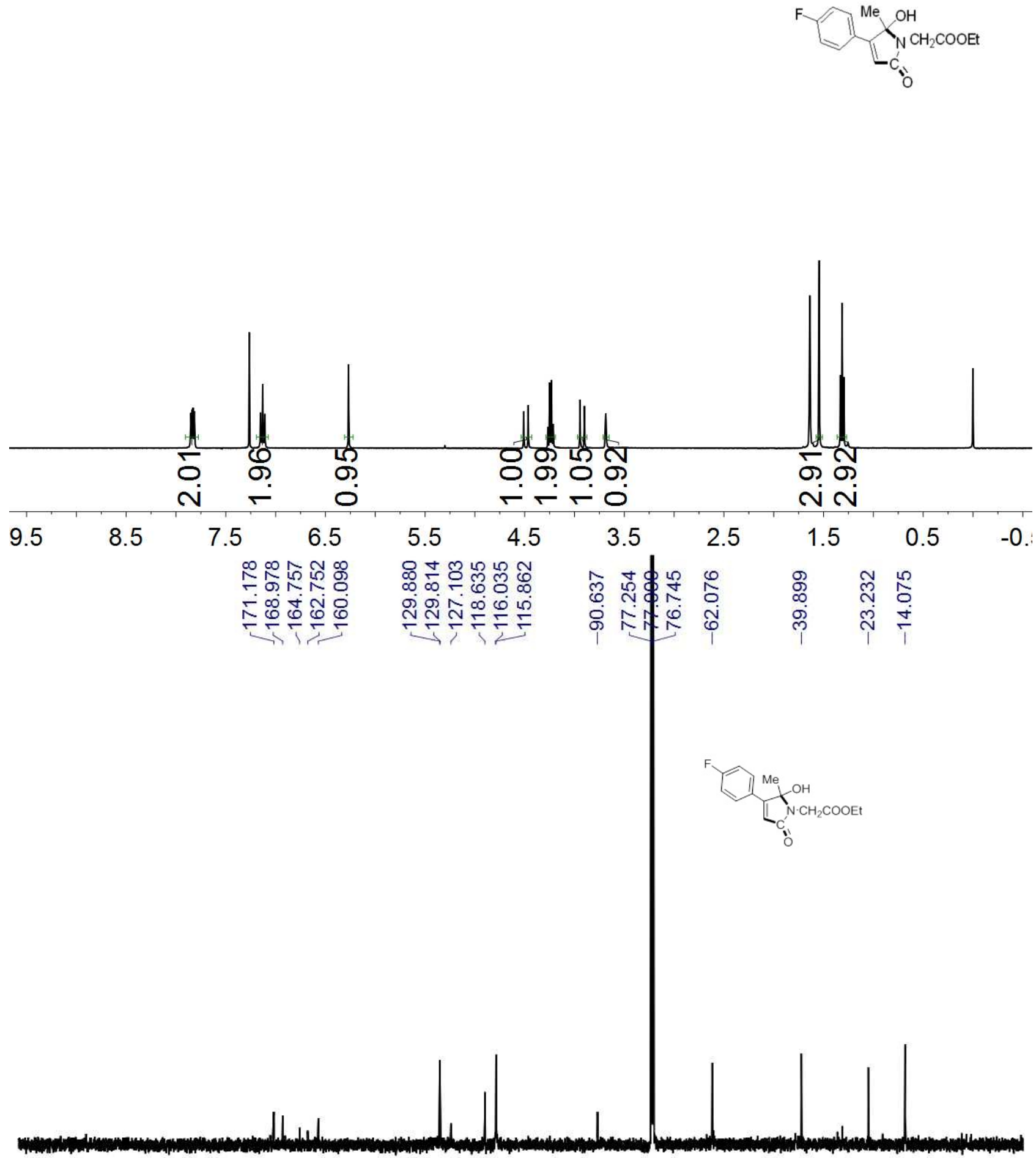

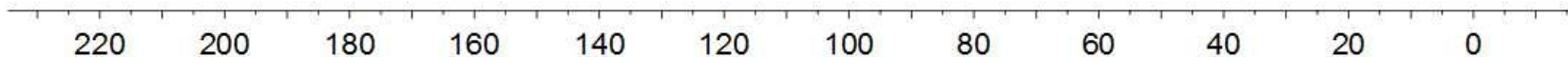




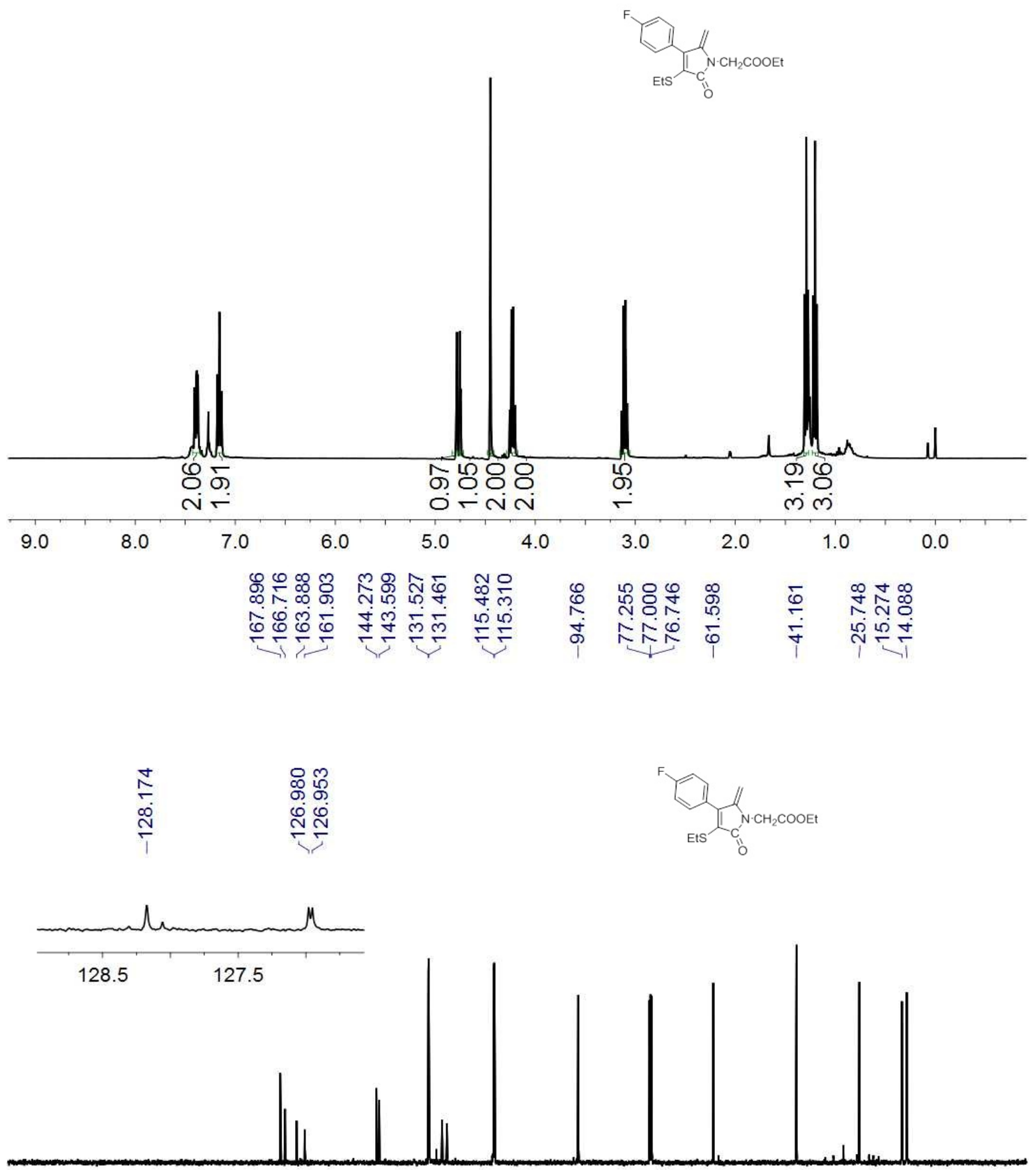




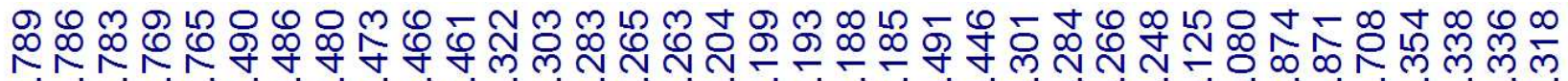

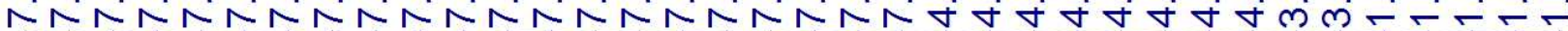

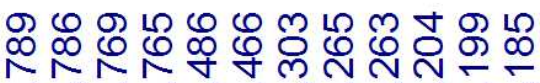

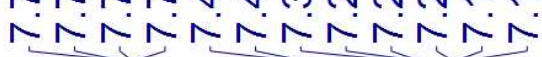
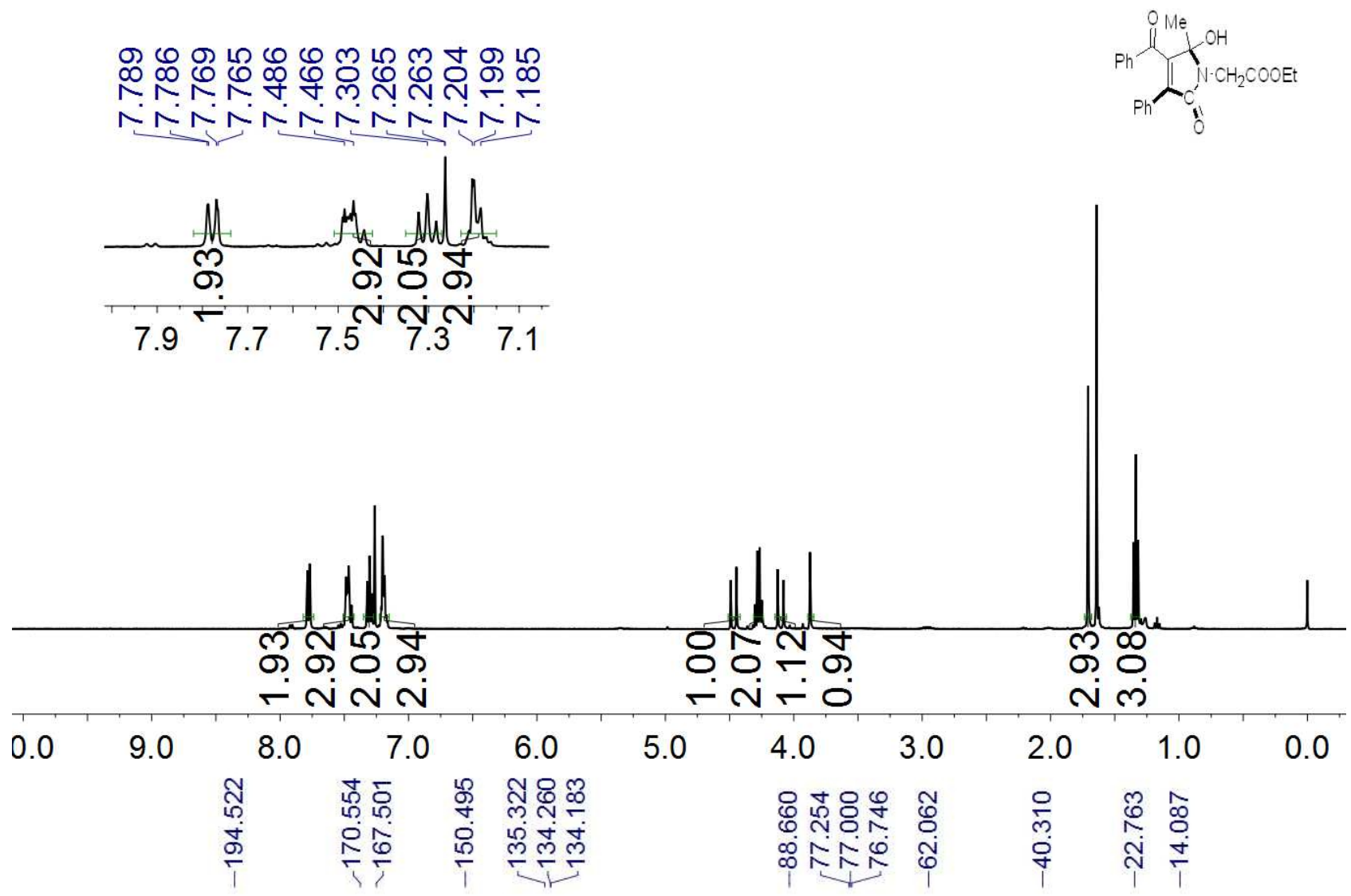

hุำ

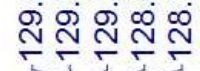
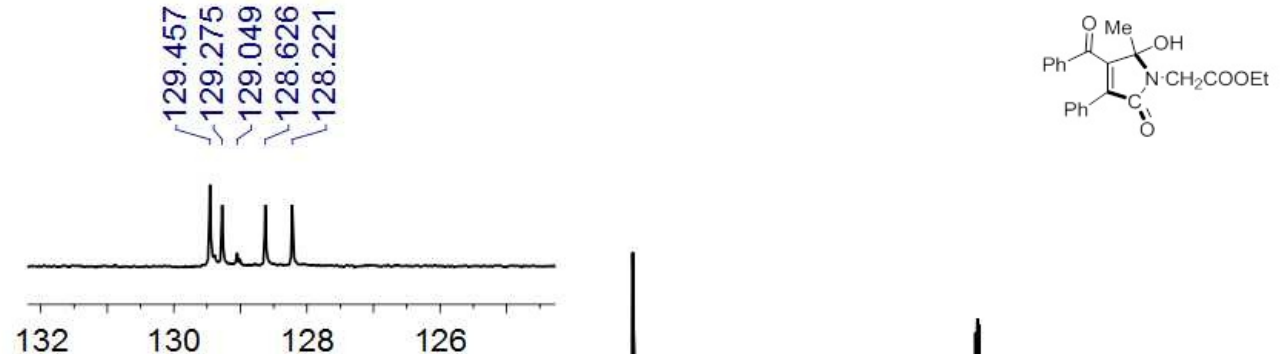

132

130

$128 \quad 126$ 


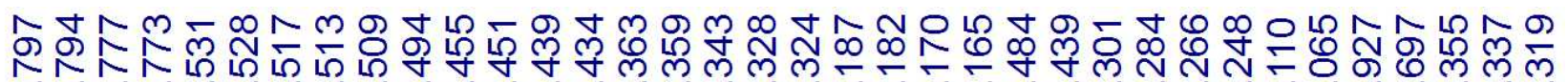

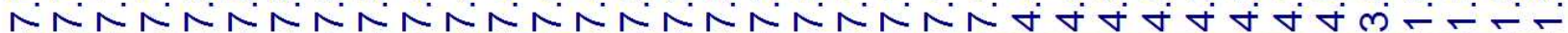

ลัง
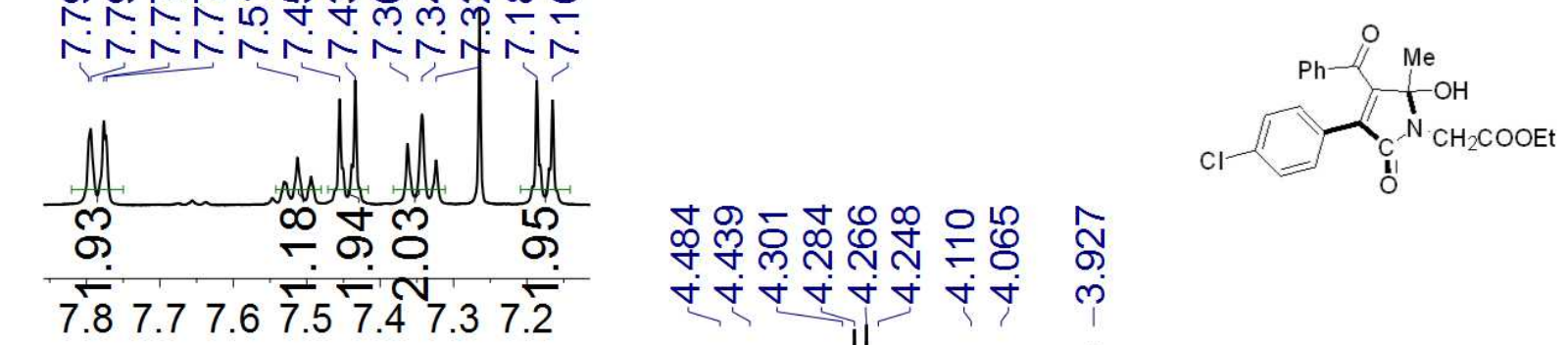

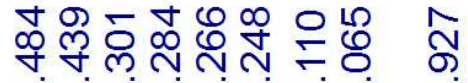
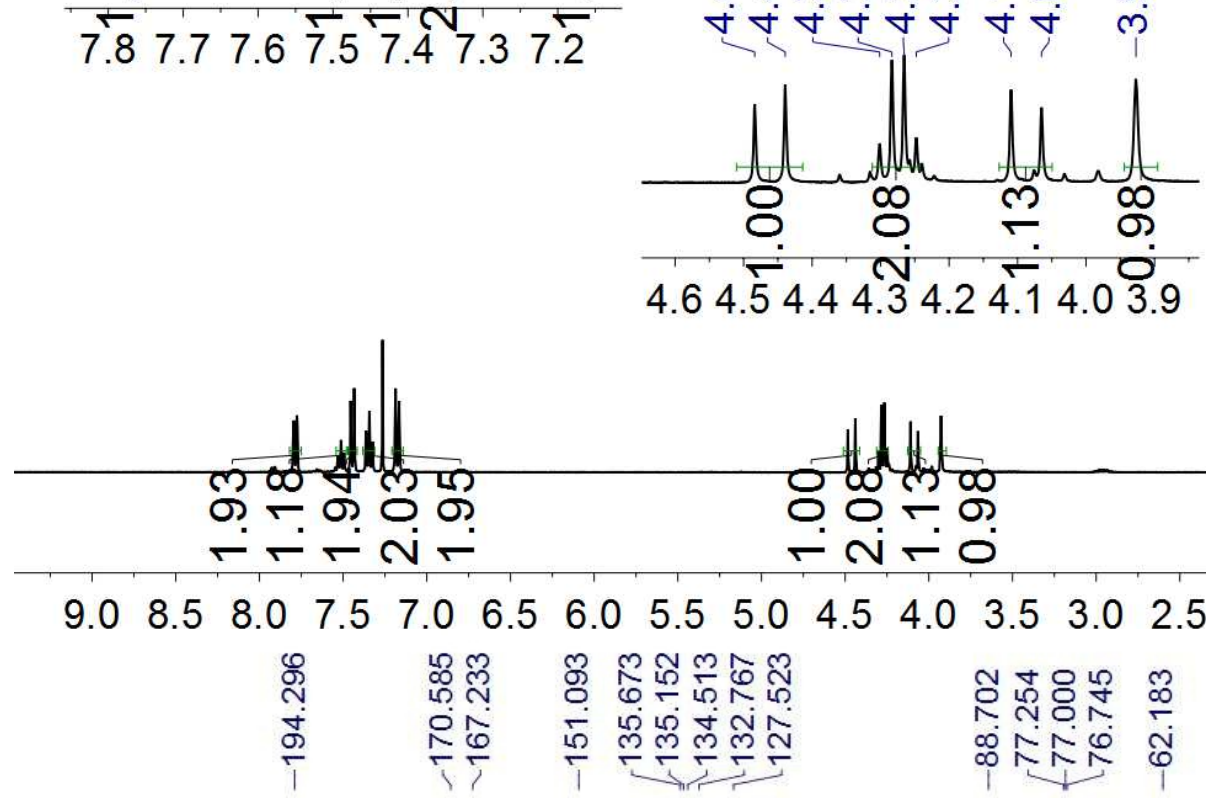

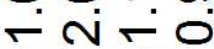

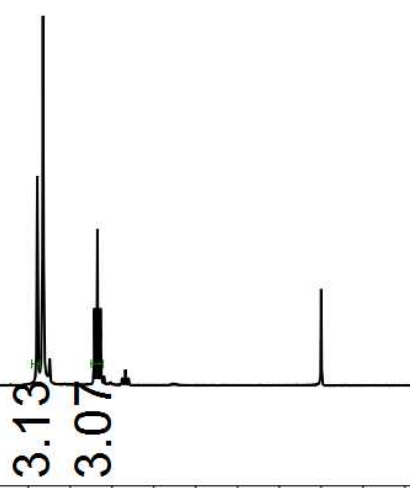

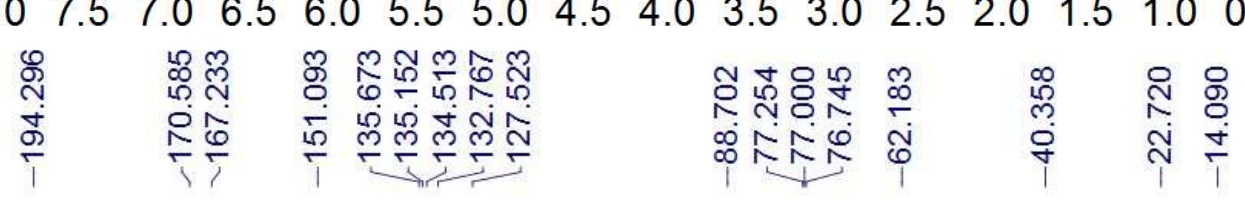
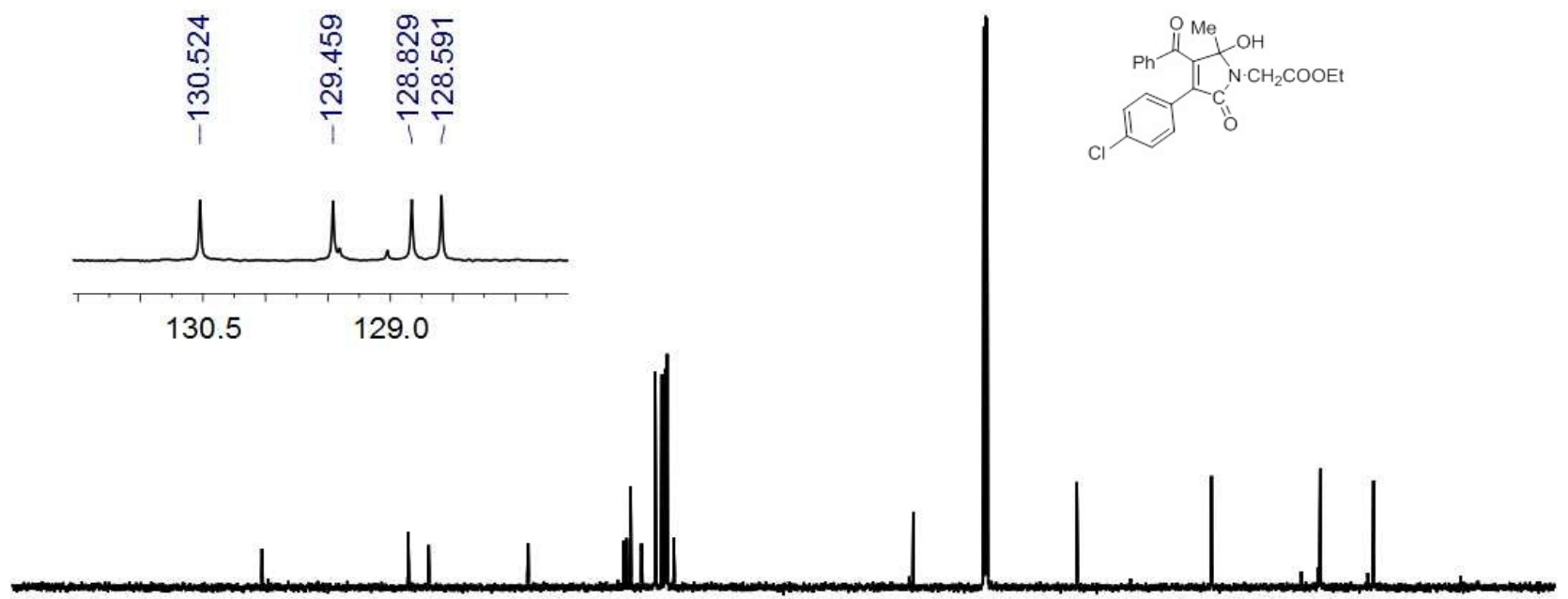

140

120

100

80

60

40

20 